Pacific Journal of Mathematic 


\section{SOME PROPERTIES OF MODULAR CONJUGATION OPERATOR OF VON NEUMANN ALGEBRAS AND A NON-COMMUTATIVE RADON-NIKODYM THEOREM WITH A CHAIN RULE}

\section{HUZIHIRO ARAKI}

For a cyclic and separating vector $\Psi$ of a von Neumann algebra $R$, the corresponding modular conjugation operator $J_{\psi}$ is characterized by the property that it is an antiunitary involution satisfying $J_{\Psi} \Psi=\Psi, J_{\Psi} R J_{\Psi}=R^{\prime}$ and $\left(\Psi, Q j_{\Psi}(Q) \Psi\right) \geqq 0$ for all $Q \in R$ where $j_{\Psi}(Q)=J_{\Psi} Q J_{\Psi}$.

The strong closure $V_{\Psi}$ of the vectors $Q j_{\Psi}(Q) \Psi$ is shown to be a $J_{\psi}$-invariant pointed closed convex cone which algebraically span the Hilbert space $H$. Any $J_{\psi \text {-invariant } \Phi \in H \text { has }}$ a unique decomposition $\Phi=\Phi_{1}-\Phi_{2}$ such that $\Phi_{j} \in V_{\Psi}$ and $s^{R}\left(\Phi_{1}\right) \perp s^{R}\left(\Phi_{2}\right)$.

There exists a unique bijective homeomorphism $\sigma_{T}$ from the set of all normal linear functionals on $R$ onto $V_{\Psi}$ such that the expectation functional by the vector $\sigma_{\Psi}(\rho)$ is $\rho$. It satisfies

$$
\begin{aligned}
& \left\|\sigma_{\Psi}\left(\rho_{1}\right)-\sigma_{\Psi}\left(\rho_{2}\right)\right\|^{2} \leqq\left\|\rho_{1}-\rho_{2}\right\| \\
& \quad \leqq\left\{\left\|\sigma_{\Psi}\left(\rho_{1}\right)+\rho_{\Psi}\left(\rho_{2}\right)\right\|\right\}\left\|\sigma_{\Psi}\left(\rho_{1}\right)-\sigma_{\Psi}\left(\rho_{2}\right)\right\| .
\end{aligned}
$$

Any two $\sigma_{T}$ and $\sigma_{\Psi}$ are related by a unitary $u^{\prime}$ in $R^{\prime}$ by $u^{\prime} \sigma_{\Psi}(\rho)=\sigma_{\Psi}(\rho)$ for all $\rho$.

The relation $l \rho_{1} \geqq \rho_{2}$ holds if and only if there exists $A\left(\rho_{2} / \rho_{1}\right) \in R$ such that $A\left(\rho_{2} / \rho_{1}\right) \sigma_{\Psi}\left(\rho_{1}\right)=\sigma_{\Psi}\left(\rho_{2}\right)$. The smallest $l$ is given by $\left\|A\left(\rho_{2} / \rho_{1}\right)\right\|$. It satisfies the chain rule $A\left(\rho_{3} / \rho_{2}\right) A\left(\rho_{2} / \rho_{1}\right)=$ $A\left(\rho_{3} / \rho_{1}\right)$. It coincides with the positive square root of the measure theoretical Radon-Nikodym derivative if $R$ is commutative.

As an application, it is shown that product of any two modular conjugation $j_{\Psi} j_{\Phi}$ is an inner automorphism of $R$.

For a product state $\otimes \rho_{j}$ of a $C^{*}$ algebra generated by finite $W^{*}$ tensor products $\left\{\bigotimes_{j \in I} R_{j}\right\} \otimes\left\{\bigotimes_{j \in I} 1_{j}\right\}$ of von Neumman algebras $R_{j}$, it is shown that $\otimes \rho_{j}$ and $\otimes \rho_{j}^{\prime}$ are equivalent if and only if $\Sigma\left\|\sigma_{\Psi}\left(\rho_{j}\right)-\sigma_{\Psi}\left(\rho_{j}^{\prime}\right)\right\|^{2}<\infty$ where $\left\|\sigma_{\Psi}(\rho)-\sigma_{\Psi}\left(\rho^{\prime}\right)\right\|$ is independent of $\Psi$.

It is shown that there exists a unitary representation $U_{\Psi}(g)$ of the group of all $*$-automorphisms of $R$ such that $U_{\Psi}(g) x U_{\Psi}(g)^{*}=g(x)$ for all $x \in R$ and $\left.\left.U_{\Psi}(g) \sigma_{\Psi}\left(g^{*} \rho\right)=\sigma_{\Psi}\right) \rho\right)$ for all normal positive linear functionals $\rho$.

1. Introduction. In the Tomita-Takesaki theory of modular automorphisms [9], two operators $\Delta_{\Psi}$ and $J_{q}$ are associated with each 
cyclic and separating vector $\Psi$ of a von Neumann algebra $R$ on a Hilbert space $H$.

$\Delta_{\Psi}$ is a positive selfadjoint operator such that

$$
\begin{gathered}
\Delta_{\Psi} \Psi=\Psi, \\
\tau_{\Psi}(t) Q \equiv\left(\Delta_{\Psi}\right)^{i t} Q\left(\Delta_{\Psi}\right)^{-i t} \in R
\end{gathered}
$$

for every $Q \in R$ and real $t$. It is called a modular operator and the automorphisms $\tau_{\Psi}(t)$ of $R$ is called modular automorphisms.

$J=J_{y}$ is an antiunitary involution, namely

$$
\begin{gathered}
(J x, J y)=(y, x) \\
J^{2}=1 .
\end{gathered}
$$

It satisfies

$$
\begin{gathered}
J \Psi=\Psi, \\
J R J=R^{\prime} .
\end{gathered}
$$

We shall call $J_{\Psi}$ a modular conjugation operator.

$\Delta_{\Psi}$ and $J_{\Psi}$ are defined through the polar decomposition

$$
\bar{S}=J_{\Psi} \Delta_{\Psi}^{1 / 2}
$$

of the closure of an antilinear operator $S$, which is defined on its domain $R \Psi$ by

$$
S Q \Psi=Q^{*} \Psi, \quad Q \in R .
$$

An important property is

$$
J_{\Psi} \Delta_{\Psi} J_{\Psi}=\Delta_{\Psi}^{-1} .
$$

Our investigation centers around the following property of $J=J_{\psi}$ observed in [2]. For any $Q \in R, Q \geqq 0, Q \neq 0$, the following strict inequality holds:

$$
\left(\Psi, Q j_{\psi}(Q) \Psi\right)>0
$$

where

$$
j_{\psi}(Q) \equiv J_{\psi} Q J_{\psi} \in R^{\prime} .
$$

The validity of (1.10) comes from the property $\Delta_{\Psi}>0$ and the following identity obtained from (1.5), (1.7), and (1.8):

$$
\left(\Psi, Q j_{\Psi}(Q) \Psi\right)=\left(Q^{*} \Psi, \Delta_{\Psi}^{1 / 2} Q^{*} \Psi\right) \text {. }
$$

Our first result is the characterization of the modular conjugation $J_{\Psi}$ for a given $\Psi$ by (1.3), (1.4), (1.5), (1.6), and (1.10). It should be 
remarked that (1.3), (1.4), (1.5), and (1.6) without (1.10) are not sufficient to characterize $J_{\psi}$. If (1.5) is dropped, then there exists a unitary $u$ in the center such that $J=J_{u \psi}$.

Our second result is concerned with the strong closure of the set of all vectors $Q j(Q) \Psi, Q \in R$. It is shown to be a pointed closed convex cone which algebraically span $H$ and is selfdual in the sense that any $\Phi \in H$ satisfying

$$
(\Phi, x) \geqq 0
$$

for all $x \in V_{\Psi}$ must be in $V_{\Psi}$. Any $\Phi \in V_{\Psi}$ is shown to have a unique decomposition $\Phi=\Phi_{1}-\Phi_{2}$, satisfying $\Phi_{1} \in V_{\Psi}, \Phi_{2} \in V_{\Psi}$ and $s^{R}\left(\Phi_{1}\right) \perp s^{R}\left(\Phi_{2}\right)$.

Our third result is concerned with a possibility of having some $\Phi \in V_{\Psi}$ for a given normal positive linear functional $\rho$ such that $\omega_{\Phi}=\rho$ where $\omega_{\Phi}$ denotes the expectation functional on $R$ by the vector $\Phi$. This turns out to be possible for all $\rho$ in a unique and nice manner. It is shown that there exists one and only one element in $V_{\psi}$-denoted as $\sigma_{\Psi} \rho$-for any given normal positive linear functional $\rho$ on $R$, such that the expectation functional $\omega_{\sigma_{\Psi} \rho}$ by the vector $\sigma_{\Psi} \rho \in V_{\Psi}$ is $\rho$. The mapping $\sigma_{\Psi}$ is bicontinuous due to the following inequality:

$$
\begin{aligned}
& \left\|\sigma_{\Psi}\left(\rho_{1}\right)-\sigma_{w}\left(\rho_{2}\right)\right\|^{2} \leqq\left\|\rho_{1}-\rho_{2}\right\| \\
& \quad \leqq\left\{\left\|\sigma_{\Psi}\left(\rho_{1}\right)+\sigma_{q}\left(\rho_{2}\right)\right\|\right\}\left\|\sigma_{\Psi}\left(\rho_{1}\right)-\sigma_{w}\left(\rho_{2}\right)\right\| .
\end{aligned}
$$

Any two $\sigma_{\Psi}$ and $\sigma_{\Psi}$, are equivalent up to a unitary equivalence, namely there exists a unitary $u^{\prime} \in R^{\prime}$ satisfying

$$
u^{\prime} \sigma_{\ddot{\Psi}}(\rho)=\sigma_{\Psi^{\prime}}(\rho)
$$

for all $\rho$.

The fourth result is concerned with the Radon-Nikodym derivative satisfying a chain rule. The relation $l \rho_{1} \geqq \rho_{2}$ for two normal positive linear functional $\rho_{1}$ and $\rho_{2}$ holds if and only if there exists $A\left(\rho_{2} / \rho_{1}\right) \in R$ such that $A\left(\rho_{2} / \rho_{1}\right) \sigma_{\psi}\left(\rho_{1}\right)=\sigma_{\psi}\left(\rho_{2}\right)$. It satisfies the chain rule

$$
A\left(\rho_{3} / \rho_{2}\right) A\left(\rho_{2} / \rho_{1}\right)=A\left(\rho_{3} / \rho_{1}\right) \text {. }
$$

If $R$ is commutative, $A\left(\rho_{2} / \rho_{1}\right)$ is the positive square root of the measure theoretical Radon-Nikodym derivative. For a general $R$, $A\left(\rho_{2} / \rho_{1}\right)$ is different from the noncommutative Radon-Nikodym derivative found by Sakai [8].

As a corollary to our investigation, we find that product of any two modular conjugation $j_{\psi} j_{\phi}$ is an inner $*$ automorphism of $R$.

Another application is made in connection with an infinite tensor product of von Neumann algebras $R_{j}$. We define

$$
d^{\prime}\left(\rho_{1}, \rho_{2}\right)=\left\|\sigma_{\psi}\left(\rho_{1}\right)-\sigma_{\psi}\left(\rho_{2}\right)\right\|
$$


which is independent of the choice of cyclic and separating vector $\Psi$. For normal states $\rho_{j}$ and $\rho_{j}^{\prime}$ of each $R_{j}$, we consider product states $\otimes \rho_{j}$ and $\otimes \rho_{j}^{\prime}$ on the $C^{*}$ algebra $A$ generated (as an inductive limit) by finite $W^{*}$ tensor products $\left\{\bigotimes_{j \in I} R_{j}\right\} \equiv R(I)$ where $I$ is any finite index set. The representations of $A$ canonically associated with $\otimes \rho_{j}$ and $\otimes \rho_{j}^{\prime}$ are quasi-equivalent if and only if

$$
\Sigma d^{\prime}\left(\rho_{j}, \rho_{j}^{\prime}\right)^{2}<\infty
$$

and the central supports of $\rho_{j}$ and $\rho_{j}^{\prime}$ are the same. The distance $d^{\prime}$ is in general larger than Bures distance [5]. They coincides if $\rho_{1}$ and $\rho_{2}$ commute.

As a further application, we show that there exists a unitary representation $U_{\Psi}(g)$ of the group of all *-automorphisms of $R$ such that $U_{\psi}(g) x U_{\Psi}(g)^{*}=g(x)$ for all $x \in R$ and $U_{\Psi}(g) \sigma_{\Psi}\left(g^{*} \rho\right)=\sigma_{\psi}(\rho)$ for all normal positive linear functionals $\rho$.

We also give a simple proof of the continuity of the modular automorphism $\tau_{\rho}(t) x$ in $\rho$ for a fixed $x \in R$ and bounded $t$.

2. A characterization of the modular conjugation operator.

THEOREM 1. Let $\Psi$ be a cyclic and separating vector for a von Neumann algebra $R$ on $H$. An operator $J$ is the modular conjugation for $\Psi$ if and only if the following 5 conditions are fulfilled.

(i ) $(J x, J y)=(y, x)$ for all $x, y \in H$.

(ii) $J^{2}=1$.

(iii) $J R J=R^{\prime}$.

(iv) $J \Psi=\Psi$.

(v) $(\Psi, Q j(Q) \Psi) \geqq 0$ for all $Q \in R$ where $j(Q) \equiv J Q J$. The equality in (v) holds if and only if $Q=0$.

Proof. It is known [9] that the modular conjugation $J_{\Psi}$ for the vector $\Psi$ satisfies (i), (ii), (iii), and (iv). (v) with the strict inequality for $Q \neq 0$ is already proved in $\S 1$.

We now prove that $J$ satisfying the 5 conditions must by $J_{\psi}$. From (i), it follows that $J$ is antilinear. From (ii), it follows that $J$ is bijective. Hence $J$ is antiunitary.

Let $T$ be defined on $R \Psi$ by

$$
T Q \Psi=J Q^{*} \Psi, \quad Q \in R .
$$

Since $\Psi$ is separating for $R, Q_{1} \Psi=Q_{2} \Psi$ implies $Q_{1}=Q_{2}$ and hence $J Q_{1}^{*} \Psi=J Q_{2}^{*} \Psi$. Therefore, $T$ is well-defined and is linear. Since $\Psi$ is cyclic for $R, T$ has a dense domain. By (iv) and (v),

$$
(Q \Psi, T Q \Psi)=\left(\Psi, Q^{*} j\left(Q^{*}\right) \Psi\right) \geqq 0, \quad Q \in R .
$$


Thus $T$ is positive on its domain and hence is symmetric.

By (1.8) and (2.1), we have

$$
T=J S .
$$

Since $J$ preserves norm, we have $\bar{T}=J \bar{S}$ and

$$
D(\bar{T})=D(\bar{S})=D\left(\Delta_{\psi}^{1 / 2}\right) .
$$

Define

$$
u \equiv J J_{\Psi} \cdot
$$

Both $J$ and $J_{w}$ are antiunitary. Hence $u$ is unitary. We have

$$
\bar{T}=u \Delta_{\psi}^{1 / 2},
$$

where (1.7) is used. We shall now show that $\bar{T}$ is selfadjoint. Then (2.2) implies that $\bar{T}$ is positive and hence (2.6) implies $\bar{T}=\Delta_{\psi}^{1 / 2}$ and $u=1$, which proves $J=J_{\Psi}$ by (2.5).

From (2.3), we have ${ }^{1}$

$$
T^{*}=S^{*} J \text {. }
$$

It is known [9] that $R^{\prime} \Psi$ is a core of $S^{*}$. (Namely, the closure of restriction of $S^{*}$ to $R^{\prime} \Psi$ is $S^{*}$.) By (iii), $J R \Psi=R^{\prime} \Psi$. Hence $R T$ is a core of $T^{*}$. Since $R \Psi$ is the domain of $T$ and $T^{*} \supset T$, we have $T^{*}=\bar{T}$.

The condition (iv) of Theorem 1 is not essential as is seen in the next result.

Theorem 2. Let $\Psi$ be cyclic and separating for $R$ in $H$. An operator $J$ satisfies conditions (i), (ii), (iii), and ( $v$ ) of Theorem 1 if and only if there exists a unitary $u$ in the center of $R$ such that

$$
J=J_{u \Psi}\left(=u J_{\Psi} u^{*}\right) .
$$

The condition (2.8) is equivalent to $J J_{\Psi}$ being in $R \cap R^{\prime}$.

For the proof we need preliminary lemmas.

LEMma 1. The weakly closed linear hull of the set of all operators $Q j(Q), Q \in R$ is $\left\{R \cup R^{\prime}\right\}^{\prime \prime}$.

Proof. For arbitrary $Q_{1} \in R$ and $Q_{2} \in R^{\prime}$, we have

$$
\begin{aligned}
Q_{3} Q_{2} & =4^{-1} \sum_{n=0}^{3} e^{i n \pi / 2} X_{n} j\left(X_{n}\right), \\
X_{n} & =Q_{1}+e^{i n \pi / 2} j\left(Q_{2}\right) \in R,
\end{aligned}
$$

${ }^{1}$ This part of proof has been simplified by a suggestion of Dr. G. Elliott. 
where $j\left(Q_{2}\right) \equiv J Q_{2} J \in R, j\left(X_{n}\right) \equiv J X_{n} J$.

Lemma 2. Let $W$ be a von Neumann algebra on $H$ such that $W^{\prime}$ is commutative. If $\Psi=\Psi_{+}+\Psi_{-}$is a cyclic vector for $W$ in $H$, and

$$
\left(\Psi_{+}, Q \Psi_{-}\right)+\left(\Psi_{-}, Q \Psi_{+}\right)=0
$$

for all $Q \in W$, then there exists a selfadjoint operator $A$ such that its spectral projections are in the center $W^{\prime}$ of $W$ and

$$
s^{W^{\prime}}\left(\Psi_{+}\right) \Psi_{-}=i A s^{W^{\prime}}\left(\Psi_{-}\right) \Psi_{+}
$$

where $s^{W \prime}\left(\Psi_{ \pm}\right)$are projections onto the closures of $W \Psi_{ \pm}$.

Proof. $s^{W^{\prime}}\left(\Psi_{ \pm}\right)$belong to $W^{\prime}$ which is commutative and hence is the center of $W$. Let

$$
E=s^{W^{\prime}}\left(\Psi_{+}\right) s^{W^{\prime}}\left(\Psi_{-}\right) .
$$

Then

$$
E \Psi_{\mp}=s^{W^{\prime}}\left(\Psi_{ \pm}\right) \Psi_{\mp} \cdot
$$

We define $A$ to be 0 on $(1-E) H$. If $E=0,(2.10)$ is trivially satisfied. Hence we consider the case $E \neq 0$.

We are going to define a selfadjoint operator $A_{1}=A E$ on $E H$ satisfying

$$
E \Psi_{-}=i A_{1} E \Psi_{+}
$$

which implies (2.10) in view of (2.11).

Since $W E \Psi_{ \pm}=E W \Psi_{ \pm}$are dense in $E s^{W^{\prime}}\left(\Psi_{ \pm}\right) H=E H, E \Psi_{ \pm}$are both cyclic for $W E$ on $E H$. Define an operator $A_{2}$ by

$$
A_{2} Q E \Psi_{+}=-i Q E \Psi_{-}, \quad Q \in W
$$

on a dense subset $W E \Psi_{+}$of $E H$.

If $Q E \Psi_{+}=0$, then (2.9), where $Q$ is replaced by $E Q_{1}^{*} Q E$, implies

$$
\begin{aligned}
\left(Q_{1} E \Psi_{+}, Q E \Psi_{-}\right) & =\left(\Psi_{+}, E Q_{1}^{*} Q E \Psi_{-}\right) \\
& =-\left(\Psi_{-}, E Q_{1}^{*} Q E \Psi_{+}\right) \\
& =0
\end{aligned}
$$

for all $Q_{1} \in W$. Therefore $Q E \Psi_{-}=0$. Hence $Q E \Psi_{+}=Q^{\prime} E \Psi_{+}$for $Q, Q^{\prime} \in W$ implies $Q E \Psi_{-}=Q^{\prime} E \Psi_{-}$, which shows that $A_{2}$ is well-defined. $A_{2}$ is obviously linear.

From (2.9), we have for $Q_{1}, Q_{2} \in W$ 


$$
\begin{aligned}
\left(Q_{1} E \Psi_{+}, A_{2} Q_{2} E \Psi_{+}\right) & =\left(\Psi_{+},-i E Q_{1}^{*} Q_{2} E \Psi_{-}\right) \\
& =\left(-i \Psi_{-}, E Q_{1}^{*} Q_{2} E \Psi_{+}\right) \\
& =\left(A_{2} Q_{1} E \Psi_{+}, Q_{2} E \Psi_{+}\right)
\end{aligned}
$$

Therefore $A_{2}$ is symmetric. $A_{2}$ obviously commutes with $Q \in W$ on its domain.

Since $\Psi$ is cyclic for $W, W E \Psi=E W \Psi$ is dense in $E H$. Hence $E \Psi_{+}+E \Psi_{-}=E \Psi$ is cyclic for $E W$ on $E H$. It is therefore separating for the commutant of $E W$ on $E H$, which is $E W^{\prime}$.

From (2.9), we have

$$
\left(E \Psi_{+}-E \Psi_{-}, Q\left(E \Psi_{+}-E \Psi_{-}\right)\right)=\left(E \Psi_{+}+E \Psi_{-}, Q\left(E \Psi,+E \Psi_{-}\right)\right) \text {. }
$$

Hence $\left\|Q\left(E \Psi_{+}-E \Psi_{-}\right)\right\|^{2}=0$ implies $\|Q E \Psi\|^{2}=0$ for any $Q \in W$. As we have seen, $E \Psi$ is separating for $E W^{\prime}$ and hence $E \Psi_{+}-E \Psi_{-}$is also separating for $E W^{\prime}$. It is therefore cyclic on $E H$ for the commutant of $E W^{\prime}$ on $E H$ which is $E W$.

Since

$$
\begin{gathered}
\left(A_{2}+i\right) Q E \Psi_{+}=i Q\left(E \Psi_{+}-E \Psi_{-}\right), \\
\left(A_{2}-i\right) Q E \Psi_{+}=-i Q\left(E \Psi_{+}+E \Psi_{-}\right),
\end{gathered}
$$

for all $Q \in W, A_{2}+i$ and $A_{2}-i$ have both dense ranges in $E H$ by cyclicity of $E \Psi_{+}-E \Psi_{-}$and $E \Psi_{+}+E \Psi_{-}$for $E W$. Therefore, the closure $A_{1}=\bar{A}_{2}$ of $A_{2}$ is selfadjoint. By (2.13) with $Q=1$, we have (2.12).

REMARK. The assumption that $\Psi$ is cyclic for $W$ can be omitted. Let $e=s^{W^{\prime}}(\Psi)$. Then $(1-e) \Psi_{+}=-(1-e) \Psi_{-}$. Substituting $Q=(1-e)$ into (2.9), we obtain

$$
\left\|(1-e) \Psi_{+}\right\|^{2}=\left\|(1-e) \Psi_{-}\right\|^{2}=0 .
$$

Hence we may restrict our attention to $e W$ on $e H$ with $\Psi, \Psi_{+}, \Psi_{-}$all in $e H$ and apply proof of Lemma 2 .

Lemma 3. If $Q \in R \cap R^{\prime}$, then

$$
J_{\psi} Q J_{\Psi}=Q^{*}
$$

where $J_{\Psi}$ is the modular conjugation operator for a cyclic and separating vector $\Psi$ of $R$.

Proof. It is known ([1], [9]) that the center of $R$ is elementwise invariant under any $K M S$ automorphisms. Hence $Q \in R \cap R^{\prime}$ commutes with $\Delta_{\Psi}$. We have 


$$
\begin{aligned}
\left(J_{\Psi} Q J_{\Psi}\right) \Psi=J_{\Psi} Q \Psi & =\Delta_{\Psi}^{1 / 2} Q^{*} \Psi \\
& =Q^{*} \Delta_{\Psi}^{1 / 2} \Psi=Q^{*} \Psi .
\end{aligned}
$$

By (iii) of Theorem 1, $J_{\psi}\left(R \cap R^{\prime}\right) J_{\psi}=R \cap R^{\prime}$. Since $\Psi$ is separating for $R \supset R \cap R^{\prime}$, we have (2.14).

Proof of Theorem 2. Assume that $J$ satisfies (i), (ii), (iii), and (v) of Theorem 1. From (i) and (ii), $J$ is an antiunitary operator. Set

$$
\Psi_{ \pm}=2^{-1}(\Psi \pm J \Psi)
$$

We have

$$
\begin{aligned}
& J \Psi_{ \pm}= \pm \Psi_{ \pm}, \\
& \Psi=\Psi_{+}+\Psi_{-} .
\end{aligned}
$$

By (2.16), we have for $Q \in R$

$$
\begin{aligned}
\left(\Psi_{ \pm}, Q j(Q) \Psi_{ \pm}\right) & =\left(J \Psi_{ \pm}, Q j(Q) J \Psi_{ \pm}\right) \\
& =\left(J \Psi_{ \pm}, J Q j(Q) \Psi_{ \pm}\right) \\
& =\overline{\left(\Psi_{ \pm}, Q j(Q) \Psi_{ \pm}\right)}
\end{aligned}
$$

where the second equality is due to $Q j(Q)=j(Q) Q$ and the last equality is due to (i). Similarly,

$$
\left(\Psi_{ \pm}, Q j(Q) \Psi_{\mp}\right)=-\overline{\left(\Psi_{ \pm}, Q j(Q) \Psi_{\mp}\right)} .
$$

Hence

$$
i \operatorname{Im}(\Psi, Q j(Q) \Psi)=\left(\Psi_{+}, Q j(Q) \Psi_{-}\right)+\left(\Psi_{-}, Q j(Q) \Psi_{+}\right) .
$$

By (v), this must vanish. By Lemma 1 , the weakly closed linear hull of $Q j(Q), Q \in R$ is $\left(R \cup R^{\prime}\right)^{\prime \prime}$. Setting $W=\left(R \cup R^{\prime}\right)^{\prime \prime}$, the premises of Lemma 2 are satisfied. Note that $W^{\prime}=R \cap R^{\prime}$ is the center of $R$ and is commutative.

Hence there exists a selfadjoint operator $A$ affiliated with $R \cap R^{\prime}$ such that (2.10) is satisfied. We define a unitary operator $u$ in $R \cap R^{\prime}$ by

$$
\begin{aligned}
u= & s^{W \prime}\left(\Psi_{+}\right)\left(1-s^{W^{\prime}}\left(\Psi_{-}\right)\right) \\
& +(1-i A)\left(1+A^{2}\right)^{-1 / 2} s^{W^{\prime}}\left(\Psi_{+}\right) s^{W^{\prime}}\left(\Psi_{-}\right) \\
& +i s^{W^{\prime}}\left(\Psi_{-}\right)\left(1-s^{W^{\prime}}\left(\Psi_{+}\right)\right) .
\end{aligned}
$$

Because $\Psi$ is cyclic for $R$, it is cyclic for $W$. Hence $s^{W^{\prime}}\left(\Psi_{+}\right) \vee s^{W^{\prime}}\left(\Psi_{-}\right) \geqq$ $s^{W^{\prime}}(\Psi)=1$. Thus

$$
\left(1-s^{W^{\prime}}\left(\Psi_{-}\right)\right)\left(1-s^{W^{\prime}}\left(\Psi_{+}\right)\right)=0
$$

and $u$ is unitary. 
From (2.10) and (2.18), we have

$$
\begin{aligned}
u \Psi= & \left(1-s^{W^{\prime}}\left(\Psi_{-}\right)\right) \Psi_{+} \\
& +\left(1+A^{2}\right)^{1 / 2} s^{W^{\prime}}\left(\Psi_{-}\right) \Psi_{+} \\
& +i\left(1-s^{W^{\prime}}\left(\Psi_{+}\right)\right) \Psi_{-} .
\end{aligned}
$$

Since $J W J=W$, both $W \Psi_{+}$and $W \Psi_{-}$are invariant under $J$. Therefore $s^{W^{\prime}}\left(\Psi_{ \pm}\right)$both commute with $J$. We shall next prove that $A$ commutes with $J$.

As we have seen, $E=s^{W^{\prime}}\left(\Psi_{+}\right) s^{W W^{\prime}}\left(\Psi_{-}\right)$commutes with $J$. From (2.16) and $J W J=W$, the domain $W E \Psi_{+}$of $A_{2}$ is invariant under $J$ and $A_{2}$ commutes with $J$. Hence the closure $A_{1}$ of $A_{2}$ commutes with $J$, because $J$ preserves norm. From the uniqueness of the spectral projections and

$$
\int \lambda d E_{\lambda}=A_{1}=J A_{1} J=\int \lambda d\left(J E_{\lambda} J\right),
$$

we have $E_{\lambda}=J E_{\lambda} J$ for all spectral projections $E_{\lambda}$ of $A_{1}$. Hence $J$ commutes with $\left(1+A^{2}\right)^{1 / 2}$.

From (2.19) and (2.16), we have

$$
J u \Psi=u \Psi .
$$

Since $u$ is in the center of $R$, it commutes with $Q j(Q), Q \in R$. Since $u$ is unitary, we have

$$
(u \Psi, Q j(Q) u \Psi)=(\Psi, Q j(Q) \Psi) \geqq 0 .
$$

By Theorem 1,

$$
J=J_{u \Psi} .
$$

Since the unitary mapping $H \rightarrow u H=H, \Psi \rightarrow u \Psi, R \rightarrow u R u^{*}=R$ brings $S_{\Psi}$ to $u S_{\Psi} u^{*}=S_{u \Psi}$, we have

$$
u J_{\psi} u^{*}=J_{u \psi} .
$$

Hence we have (2.8).

By Lemma 3, we have

$$
J J_{\Psi}=u J_{\Psi} u^{*} J_{\Psi}=u^{2}
$$

which is a unitary operator in the center of $R$.

Conversely, let $w$ be a unitary operator in $R \cap R^{\prime}$ and $J J_{Y}=w$. Then $J=w J_{\Psi}$ satisfies (i), (ii), (iii), and (v) of Theorem 1, where (ii) is due to Lemma 3:

$$
\left(w J_{\Psi}\right)^{2}=w J_{\Psi} w J_{\Psi}=w w^{*}=1
$$

The following example shows the case where (i), (ii), (iii), and (iv) 
are satisfied but $J \neq J_{\Psi}$. The center in this example is trivial and $J \neq u J_{\psi} u^{*}$ for any unitary $u$ in the center.

ExAmple. Let $H_{n}$ be $n$ dimensional Hilbert space and $R=B\left(H_{2}\right) \otimes 1$ be the algebra of $2 \times 2$ matrices on $H_{4}=H_{2} \otimes H_{2}$. Let $e_{1}, e_{2}$ be an orthonormal basis of $H_{2}$ and $e_{i j}=e_{i} \otimes e_{j} \in H_{4}$. Let $\Psi=2^{-1 / 2}\left(e_{11}+e_{22}\right)$, $\Phi=2^{-1 / 2}\left(e_{12}+e_{21}\right)$. Then $J_{\dddot{t}} e_{i j}=e_{j i}$ while $J_{\phi} e_{i j}=e_{i j}$ for $i \neq j$ and $J_{\emptyset} e_{i i}=e_{j j}$ for $i \neq j$. Hence $J_{\varphi} \neq J_{\phi}$. However, $J=J_{\emptyset}$ satisfies (i), (ii), and (iii) because it is a modular conjugation operator for $\Phi$ and satisfies (iv).

REMARK. The condition (iii) is used only in the proof of the essential selfadjointness in Theorem 1 . If $R$ is a finite matrix algebra then (i), (ii), (iv), and (v) are sufficient to prove $J=J_{\dddot{y}}$. Whether (iii) is necessary for more general case is an open question.

3. Technical lemmas concerning $\Delta_{\Psi}^{z} Q \Delta_{\Psi}^{-z}$. We denote by $\mathfrak{U}_{\Psi}$ the set of all operators $Q$ such that there exists a family of bounded linear operators $\tau_{\Psi}(z) Q$ depending on a complex parameter $z$, which is holomorphic in $z$ for all $z$ and satisfies

$$
\tau_{\Psi}(t) Q=\Delta_{\Psi}^{i t} Q \Delta_{\Psi}^{-i t}
$$

for real $t$.

For real $z$, we have

$$
\tau_{\Psi}(z) Q \Delta_{\Psi}^{i z} \Phi=\Delta_{\Psi}^{i z} Q \Phi, \quad \Phi \in D\left(\Delta_{\Psi}^{i z}\right) .
$$

If $\Phi$ is an entire vector of $\log \Delta_{\Psi}$, then the left hand side is an entire function of $z$ and hence $Q \Phi$ must be an entire vector of $\log \Delta_{\psi}$ and (3.2) holds for all $z$. Since vectors, on which $\log \Delta_{w}$ is bounded, are entire vector of $\log \Delta_{\Psi}$ and form a dense set of analytic vectors for $\Delta_{\psi}^{\alpha}$ for any real $\alpha$, (3.2) holds for any $z$ and $\Phi \in D\left(\Delta_{\Psi}^{i z}\right)$ by Nelson's theorem.

If $Q_{1}$ and $Q_{2}$ are in $\mathfrak{N}_{\Psi}$, then $\left(\tau_{\Psi}(z) Q_{1}\right) \tau_{\Psi}(z) Q_{2}$ is an entire function of $z$ and satisfies (3.1) for $Q=Q_{1} Q_{2}$. Hence $Q_{1} Q_{2} \in \mathfrak{A}_{\psi}$ and

$$
\tau_{y}(z)\left(Q_{1} Q_{2}\right)=\left\{\tau_{\psi}(z) Q_{1}\right\} \tau_{\psi}(z) Q_{2} .
$$

Similarly, $Q \in \mathfrak{V}_{\Psi}$ implies $Q^{*} \in \mathfrak{N}_{\Psi}$ and

$$
\tau_{\Psi}(z)\left(Q^{*}\right)=\left(\tau_{\Psi}(\bar{z}) Q\right)^{*} .
$$

We define

$$
\begin{array}{ll}
\mathfrak{U}_{\Psi_{1}}=\mathfrak{U}_{\Psi} \cap R, & D_{\Psi_{1}}=\mathfrak{U}_{1 \Psi} \Psi, \\
\mathfrak{U}_{\Psi_{2}}=\mathfrak{U}_{\Psi} \cap R^{\prime}, & D_{\Psi_{2}}=\mathfrak{U}_{\Psi_{2}} \Psi .
\end{array}
$$


If $Q \in \mathfrak{A}_{\psi_{1}}$, then $\left[\tau_{\Psi}(z) Q, Q_{1}\right]=0$ for any $Q_{1} \in R^{\prime}$ and real $z$, hence for all $z$ by an analytic continuation. Therefore $\tau_{\Psi}(z) Q \in \mathfrak{A}_{\Psi_{1}}$. Similarly, if $Q \in \mathfrak{A}_{\Psi_{2}}$, then $\tau_{\Psi}(z) Q \in \mathfrak{A}_{\Psi_{2}}$ for all $z$.

For any $L^{1}$ function $f$, we define

$$
Q(f)=\int \Delta_{\Psi}^{i t} Q \Delta_{\Psi}^{-i t} f(t) \mathrm{d} t
$$

It is bounded $\left(\|Q(f)\| \leqq\|Q\| \int|f(t)| \mathrm{d} t\right), Q(f) \in R$ if $Q \in R$ and $Q(f) \in R^{\prime}$ if $Q \in R^{\prime}$. If $\tilde{f}$ is a $C^{\infty}$ function such that $e^{\alpha \lambda} \tilde{f}(\lambda)$ is bounded for any real $\alpha$, and

$$
f(t)=(2 \pi)^{-1} \int e^{-i \lambda t} \widetilde{f}(\lambda) \mathrm{d} \lambda
$$

then $Q(f) \in \mathfrak{N}_{\psi}$ and

$$
\begin{gathered}
\tau_{y}(z) Q(f)=Q\left(f_{z}\right), \\
f_{z}(t)=(2 \pi)^{-1} \int e^{-i \lambda(t-z)} \tilde{f}(\lambda) \mathrm{d} \lambda .
\end{gathered}
$$

We shall use the following specific function later:

$$
f_{\beta}^{G}(t)=(\beta \pi)^{-1 / 2} \exp \left\{-t^{2} / \beta\right\}, \quad \beta>0 .
$$

It has the property that $Q\left(f_{\beta}^{G}\right)$ is in the weak closure of convex hull of $\Delta_{\psi}^{i t} Q \Delta_{\Psi}^{-i t}$ and

$$
\lim _{\beta \rightarrow 0} Q\left(f_{\beta}^{G}\right)=Q .
$$

If $\widetilde{f}$ has a compact support, then $Q(f) \Psi$ is an analytic vector of $\Delta_{\Psi}^{\alpha}$ for any real $\alpha$. Since

$$
Q(f) \Psi=\tilde{f}\left(\log \Delta_{\Psi}\right) Q \Psi
$$

and $R \Psi$ is dense, such vectors $Q(f) \Psi$ are dense and hence $D_{\Psi_{1}}$ is a core of $\Delta_{\Psi}^{z}$ for arbitrary $z$. Similarly, $D_{\psi_{2}}$ is also a core of $\Delta_{\Psi}^{z}$ for arbitrary $z$.

LEMMA 4. Let $Y=\int \lambda \mathrm{d} p_{\lambda}$ be a positive selfadjoint operator and $D$ be a core of $Y$. Then $D$ is a core of $Y^{\alpha}$ for $0 \leqq \alpha \leqq 1$.

Proof. Any vector in the domain of $Y$ is in the domain of $Y^{\alpha}$, $0 \leqq \alpha \leqq 1$. Then

$$
\begin{aligned}
\left\|Y^{\alpha} x\right\|^{2} & =\left\|p_{1} Y^{\alpha} x\right\|+\left\|\left(1-p_{1}\right) Y^{\alpha} x\right\|^{2} \\
& \leqq\left\|p_{1} x\right\|^{2}+\left\|\left(1-p_{1}\right) Y x\right\|^{2} \\
& \leqq\|x\|^{2}+\|Y x\|^{2} .
\end{aligned}
$$


If $x_{n} \in D, x_{n} \rightarrow x \in D(Y)$ and $Y x_{n} \rightarrow Y x$, then $Y^{\alpha} x_{n}$ is Cauchy by (3.13) and hence $x \in D\left(\left(Y^{\alpha} \mid D\right)^{-}\right)$. Since $D(Y)$ is a core of $Y^{\alpha}, 0 \leqq \alpha \leqq 1, D$ is also a core of $Y^{\alpha}$.

LEMma 5. For $Q \in R$, the following two conditions are equivalent.

$$
\begin{gathered}
Q \Psi \in D\left(\Delta_{\Psi}^{(1 / 2)+\alpha}\right) . \\
Q^{*} \Psi \in D\left(\Delta_{\Psi}^{-\alpha}\right) .
\end{gathered}
$$

If these conditions are satisfied for an $\alpha>0$, then there exists a family of closable operators $\hat{\tau}_{w}(z) Q$ for $\operatorname{Im} z \in[-\alpha, 0]$ with a common domain $D_{\Psi_{2}}$ such that

(1) $\hat{\tau}_{w}(z) Q$ is affiliated with $R$,

(2) $\hat{\tau}_{w}(z) Q x$ is continuous in $z$ for $\operatorname{Im} z \in[-\alpha, 0]$ and analytic in $z$ for $z \in[-\alpha, 0)$ if $x \in D_{\Psi_{2}}$,

(3) $\hat{\tau}_{\Psi}(z) Q x=\Delta_{\Psi}^{i z} Q \Delta_{\Psi}^{-i z} x, x \in D_{\Psi 2}$,

(4) $\left(\hat{\tau}_{\Psi}(z) Q\right)^{*} x=\Delta_{\psi}^{i \bar{z}} Q^{*} \Delta_{\bar{\psi}}^{-i \bar{z}} x, x \in D_{\Psi_{2}}$.

Proof. Due to $J_{\Psi} \Delta_{\Psi}^{\alpha}=\Delta_{\Psi}^{-\alpha} J_{\Psi}$, we have

$$
D\left(\Delta_{\bar{\Psi}}^{-\alpha}\right)=J_{\Psi} D\left(\Delta_{\Psi}^{\alpha}\right) \text {. }
$$

Hence (3.15) is equivalent to

$$
\Delta_{\Psi}^{1 / 2} Q \Psi=J_{\Psi} Q^{*} \Psi \in D\left(\Delta_{\Psi}^{\alpha}\right)
$$

which is equivalent to (3.14).

Assume that $Q$ satisfies (3.14) and (3.15). Define an operator $A_{z}$ on $D_{\Psi_{2}}$ by

$$
A_{z} Q^{\prime} \Psi=Q^{\prime} \Delta_{\Psi}^{i z} Q \Psi, \quad Q^{\prime} \in \mathfrak{A}_{\Psi_{2}},
$$

where $\operatorname{Im} z \in[-\alpha, 0]$. By (3.14), $Q \Psi$ is in the domain of $\Delta_{\psi}^{i z}$ for $\operatorname{Im} z \in[-\alpha, 0]$. Since $\Psi$ is separating for $R^{\prime} \supset \mathfrak{A}_{\Psi_{2}}, A_{z}$ is well-defined and linear.

To show that $A_{z}$ is closable, we show that its adjoint has a dense domain. For $Q_{1}^{\prime}$ and $Q_{2}^{\prime}$ in $\mathfrak{A}_{\Psi_{2}}$, we have

$$
\begin{aligned}
\left(Q_{1}^{\prime} \Psi, A_{z} Q_{2}^{\prime} \Psi\right) & =\left(Q_{2}^{*} Q_{1}^{\prime} \Psi, \Delta_{\Psi}^{i z} Q \Psi\right) \\
& =\left(\Delta_{\Psi}^{-1 / 2}\left\{\tau(-\bar{z}-i / 2)\left(Q_{2}^{\prime *} Q_{1}^{\prime}\right)\right\} \Psi, Q \Psi\right) \\
& =\left(J_{\Psi} \Delta_{\Psi}^{-i z-1 / 2} Q_{1}^{* *} Q_{2}^{\prime} \Psi, J_{\Psi} \Delta_{\Psi}^{1 / 2} Q^{*} \Psi\right) \\
& =\left(\Delta_{\Psi}^{1 / 2} Q^{*} \Psi, \Delta_{\Psi}^{-i z-1 / 2} Q_{1}^{\prime *} Q_{2}^{\prime} \Psi\right) \\
& =\left(Q_{1}^{\prime} \Delta_{\Psi}^{i z} Q^{*} \Psi, Q_{2}^{\prime} \Psi\right)
\end{aligned}
$$

where $Q^{*} \Psi$ is in the domain of $\Delta_{\psi}^{i z}$ by (3.15). This proves that $D\left(A_{z}^{*}\right)$ contains a dense set $D_{\Psi_{2}}$ and $A_{z}$ is closable. We denote $A_{z}=\hat{\tau}_{\Psi}(z) Q$. 
(1) By (3.17), we have

$$
Q_{1}^{\prime} A_{z} Q_{2}^{\prime} \Psi=Q_{1}^{\prime} Q_{2}^{\prime} \Delta_{\Psi}^{i z} Q \Psi=A_{z} Q_{1}^{\prime} Q_{2}^{\prime} \Psi
$$

for any $Q_{1}^{\prime}$ and $Q_{2}^{\prime}$ in $\mathfrak{A}_{\Psi_{2}}$. Hence $A_{z}$ commutes with $Q_{1}^{\prime} \in \mathfrak{A}_{\Psi_{2}}$ and is affiliated with $\left(\mathfrak{A}_{\Psi_{2}}\right)^{\prime}=R$.

(2) By (3.17), we have

$$
\left(\widehat{\tau}_{\Psi}(z) Q\right) Q^{\prime} \Psi=Q^{\prime} \triangle_{\Psi}^{i z} Q \Psi
$$

which has the stated continuity and analyticity due to (3.14).

(3) This follows from the following computation:

$$
\begin{aligned}
\Delta_{\Psi}^{i z} Q \Delta_{\Psi}^{-i z} Q^{\prime} \Psi & =\Delta_{\Psi}^{i z} Q\left\{\tau_{\Psi}(-z) Q^{\prime}\right\} \Psi \\
& =\Delta_{\Psi}^{i z}\left\{\tau_{\Psi}(-z) Q^{\prime}\right\} Q \Psi \\
& =Q^{\prime} \Delta_{\Psi}^{i z} Q \Psi=A_{z} Q^{\prime} \Psi .
\end{aligned}
$$
used.

(4) This follows from the following computation where (3.18) is

$$
\begin{aligned}
\left(Q_{1}^{\prime} \Psi,\left(\hat{\tau}_{\Psi}(z) Q\right) Q_{2}^{\prime} \Psi\right) & =\left(Q_{1}^{\prime} \Delta_{\Psi}^{i z} Q^{*} \Psi, Q_{2}^{\prime} \Psi\right) \\
& =\left(\Delta_{\Psi}^{i z}\left\{\tau_{\Psi}(-\bar{z}) Q_{1}^{\prime}\right\} Q^{*} \Psi, Q_{2}^{\prime} \Psi\right) \\
& =\left(\Delta_{\Psi}^{i z} Q^{*}\left\{\tau_{\Psi}(-\bar{z}) Q_{1}^{\prime}\right\} \Psi, Q_{2}^{\prime} \Psi\right) \\
& =\left(\Delta_{\Psi}^{i \bar{z}} Q^{*} \Delta_{\Psi}^{-i \bar{z}} Q_{1}^{\prime} \Psi, Q_{2}^{\prime} \Psi\right) .
\end{aligned}
$$

CoRollary. For $Q \in R$, the following two conditions are equivalent.

$$
\begin{gathered}
Q \Psi \in D\left(\Delta_{\Psi}^{-\alpha}\right) . \\
Q^{*} \Psi \in D\left(\Delta_{\Psi}^{(1 / 2)+\alpha}\right) .
\end{gathered}
$$

If these conditions are satisfied for an $\alpha>0$, then there exists a family of closable operators $\hat{\tau}_{y}(z) Q$ for $\operatorname{Im} z \in[0, \alpha]$ with a common domain $D_{\Psi_{2}}$ such that

(1) $\hat{\tau}(z) Q$ is affliated with $R$,

(2) $\hat{\tau}_{y}(z) Q x$ is continuous in $z$ for $\operatorname{Im} z \in[0, \alpha]$ and analytic in $z$ for $\operatorname{Im} z \in(0, \alpha)$ if $x \in D_{\Psi_{2}}$,

(3) $\hat{\tau}_{\Psi}(z) Q x=\Delta_{\Psi}^{i z} Q \Delta_{\Psi}^{-i z} x, x \in D_{\Psi_{2}}$,

(4) $\left(\hat{\tau}_{\Psi}(z) Q\right)^{*} x=\Delta_{\Psi}^{i \bar{z}} Q^{*} \Delta_{\Psi}^{i z} x, x \in D_{\psi_{2}}$.

Proof. Interchange roles of $Q$ and $Q^{*}$ in Lemma 5 and denote the restriction of $\left\{\hat{\tau}_{\Psi}(\bar{z})\left(Q^{*}\right)\right\}^{*}$ to $D_{\Psi_{2}}$ by $\hat{\tau}_{\Psi}(z) Q$. The only change is in the analyticity at the boundary $\operatorname{Im} z=\alpha$.

Lemma 6. Assume that $Q \in R$ and

$$
\Delta^{\alpha} Q \Psi=Q_{1} \Psi
$$


for some $Q_{1} \in R$ and a real $\alpha \neq 0$. Then there exists a family of operators $\tau_{\Psi}(z) Q \in R$ for $\operatorname{Im} z$ between 0 and $-\alpha$ (i.e., in $[0,-\alpha]$ if $\alpha<0$ and $[-\alpha, 0]$ if $\alpha>0$ ) such that

(1) $\tau_{\Psi}(z) Q$ is strongly continuous in $z$ for $\operatorname{Im} z \in[0,-\alpha]$ or $[-\alpha, 0]$ and analytic in $z$ for $\operatorname{Im} z \in(0,-\alpha)$ or $(-\alpha, 0)$.

(2) $\tau_{\Psi}(z) Q x=\Delta_{\Psi}^{i z} Q \Delta_{\Psi}^{-i z} x, x \in D\left(\Delta_{\bar{\Psi}}^{i z}\right)$.

(3) $\left(\tau_{\Psi}(z) Q\right)^{*} x=\Delta_{\Psi}^{i z} Q^{*} \Delta_{\bar{\psi}}^{-i \bar{z}} x, x \in D\left(\Delta_{\Psi}^{i z}\right)$.

(4) $\left\|\tau_{\Psi}(z) Q\right\| \leqq \max \left\{\|Q\|,\left\|Q_{1}\right\|\right\}$.

(5) $\tau_{\Psi}(0) Q=Q, \tau_{\Psi}(-i \alpha) Q=Q_{1}$.

Proof. First assume $\alpha>0$. Since $Q_{1} \Psi \in D\left(\Delta_{\Psi}^{1 / 2}\right)$ for any $Q_{1} \in R$, (3.21) implies (3.14). Consider

$$
f(z) \equiv\left(x, \hat{\tau}_{\Psi}(z) Q y\right)
$$

for $x, y \in D_{\Psi_{2}}$. If $x=Q_{1}^{\prime} \Psi, y=Q_{2}^{\prime} \Psi$, then

$$
\begin{aligned}
|f(z)| & =\left|\left(Q_{2}^{\prime *} Q_{1}^{\prime} \Psi, \Delta_{\Psi}^{i z} Q \Psi\right)\right| \\
& \leqq\left\|Q_{2}^{\prime *} Q_{1}^{\prime} \Psi\right\|\left\|\Delta_{\Psi}^{\operatorname{Im} z} Q \Psi\right\| \\
& \leqq\left\|Q_{2}^{\prime *} Q_{1}^{\prime} \Psi\right\|\left\{\left\|\Delta_{\Psi}^{\alpha} Q \Psi\right\|^{2}+\|Q \Psi\|^{2}\right\}^{1 / 2}
\end{aligned}
$$

for $\operatorname{Im} z \in[-\alpha, 0]$ due to (3.13). Since $f(z)$ is continuous for $\operatorname{Im} z \in$ $[-\alpha, 0]$ and is holomorphic for $\operatorname{Im} z \in(-\alpha, 0)$, the three line theorem is applicable.

On the boundary $\operatorname{Im} z=0$, we have

$$
|f(t)| \leqq\|x\|\|y\|\|Q\|, \quad t \text { real. }
$$

For $z=s-i \alpha$, we have

$$
\begin{aligned}
\left\{\hat{\tau}_{\Psi}(z) Q\right\} Q^{\prime} \Psi & =Q^{\prime} \Delta_{\Psi}^{i z} Q \Psi \\
& =Q^{\prime} \Delta_{\psi}^{i s} Q_{1} \Psi=\left\{\Delta_{\psi}^{i s} Q_{1} \Delta_{\Psi}^{-i s}\right\} Q^{\prime} \Psi .
\end{aligned}
$$

Hence

$$
|f(s-i \alpha)| \leqq\|x\|\|y\|\left\|Q_{1}\right\|, \quad s \text { real. }
$$

Therefore,

$$
|f(z)| \leqq\|x\|\|y\| \max \left\{\left\|Q_{1}\right\|,\|Q\|\right\} \text {. }
$$

This implies that $\hat{\tau}(z) Q, \operatorname{Im} z \in[-\alpha, 0]$ is bounded. We denote its closure by $\tau(z) Q$. It satisfies (4) due to the above estimate. (5) follows from definition. From (1) of Lemma $5, \tau(z) Q \in R$. Since $D_{\Psi_{2}}$ is a core of $J_{\psi}^{i z}$ for any $z$, we have (2) and (3) from (3) and (4) of Lemma 5 .

(1) holds on a dense set $D_{\mathscr{q}_{2}}$ by (2) of Lemma 5 . Due to the uniform boundedness (4), the continuity statement holds on any vector. Then analyticity statement also holds on any vector by Cauchy integral theorem. 
The proof for the case $\alpha<0$ is the same as the case $\alpha>0$. vectors

4. The cone $V_{\Psi *}^{\alpha}$. Let $V_{\Psi}^{\alpha}$ be the weak closure of the set of

$$
\left\{\triangle_{\Psi}^{\alpha} Q \Psi ; Q \in R, Q \geqq 0\right\}
$$

where $\alpha \in[0,1 / 2] . \quad V_{\Psi}^{0}$ is $\mathscr{P}^{\sharp}$ of Takesaki [9]. Since $\Delta_{\Psi}^{1 / 2} Q \Psi=J_{\Psi} Q \Psi=$ $j_{\Psi}(Q) \Psi$ for $Q \in R, Q \geqq 0, V_{\Psi}^{1 / 2}$ is $\mathscr{P}^{b}$ of Takesaki.

\section{THEOREM 3.} $\Delta_{\Psi}^{i t}$.

(1) $V_{\Psi}^{\alpha}$ is a pointed weakly closed convex cone invariant under

(2) $\Phi \in V_{\Psi}^{\alpha}$ is in the domain of $\Delta_{\Psi}^{1 / 2-2 \alpha}$ and

$$
J_{\Psi} \Phi=\Delta_{\Psi}^{1 / 2-2 \alpha} \Phi .
$$

(3) $\Delta_{\Psi}^{\alpha} V_{\Psi}^{0}$ is a dense subset of $V_{\Psi}^{\alpha}$.

(4) $J_{\Psi} V_{\Psi}^{\alpha}=V_{\Psi}^{1 / 2-\alpha}$.

(5) The dual of $V_{\Psi}^{\alpha}$ is $V_{\Psi}^{1 / 2-\alpha}$.

( 6 ) $V_{\Psi}^{\alpha}=\Delta_{\Psi}^{\alpha-1 / 4}\left\{V_{\Psi}^{1 / 4} \cap D\left(\Delta_{\Psi}^{\alpha-1 / 4}\right)\right\}$.

(7) If $Q \in R$ and $Q \Psi \in V_{\Psi}^{\alpha}$, then $\triangle_{\Psi}^{i z} Q \Delta_{\Psi}^{-i z}$ is bounded by $\|Q\|$ for $\operatorname{Im} z \in[0,2 \alpha]$ and satisfies

$$
\begin{gathered}
\left(\Delta_{\Psi}^{-2 \alpha} Q \Delta_{\Psi}^{2 \alpha}\right)^{-}=Q^{*}, \\
\left(\Delta_{\Psi}^{-\alpha} Q \Delta_{\Psi}^{\alpha}\right)^{-} \geqq 0,
\end{gathered}
$$

where the bar indicates the closure.

Conversely, if $\Delta_{\bar{T}}^{-\alpha} Q \Delta_{\Psi}^{\alpha}$ is a positive bounded operator with a dense domain affiliated with $R$, then $Q \Psi \in V_{\Psi}^{\alpha}$.

(8) If $\Phi \in V_{\Psi}^{\alpha}, \alpha \leqq 1 / 4$ and $\omega_{\Phi} \leqq l \omega_{\Psi}$ for some $l>0$, then there exists $Q \in R$ such that

$$
\Phi=Q \Psi, \quad\|Q\| \leqq l^{1 / 2},
$$

$\left(\Delta_{\psi}^{i z} Q \Delta_{\psi}^{-i z}\right)^{-}$is bounded by $l^{1 / 2}$ for $\operatorname{Im} z \in[2 \alpha-1 / 2,1 / 2]$.

(9) If $Q \Psi \in V_{\Psi}^{\alpha}, Q \in R$, then $(\|Q\|-Q) \Psi \in V_{\Psi}^{\alpha}$.

Proof. $\quad V_{\Psi}^{\alpha}$ is obviously a weakly closed convex cone. Since

$$
\Delta_{\Psi}^{i t}\left(\Delta_{\Psi}^{\alpha} Q \Psi\right)=\Delta_{\Psi}^{\alpha} Q_{t} \Psi, \quad Q_{t} \equiv \Delta_{\Psi}^{i t} Q \Delta_{\Psi}^{-i t},
$$

and $Q_{t} \in R, Q_{t} \geqq 0, V_{\Psi}^{\alpha}$ is invariant under $\Delta_{\psi}^{i t}$.

We shall prove that $V_{\Psi}^{\alpha}$ is pointed after (6).

(2) If $Q \in R, Q \geqq 0$, we have

$$
\begin{aligned}
J_{y}\left(\Delta_{\Psi}^{\alpha} Q \Psi\right) & =\Delta_{\Psi}^{-\alpha} J_{\Psi} Q \Psi=\Delta_{\Psi}^{1 / 2-\alpha} Q \Psi \\
& =\Delta_{\Psi}^{(1 / 2-2 \alpha)}\left(\Delta_{\Psi}^{\alpha} Q \Psi\right) .
\end{aligned}
$$


Hence $\Delta_{\psi}^{\alpha} Q \Psi$ satisfies (4.2).

Since $J_{\Psi}$ is bounded and $\Delta_{\Psi}^{(1 / 2-2 \alpha)}$ is closed, (4.2) holds for any $\Phi$ in the strong closure of the set (4.1). Since the set (4.1) is convex, its strong and weak closures coincide.

(3) Since (4.1) is convex, $V_{\Psi}^{\alpha}$ is the strong closure of (4.1). If $\Phi \in V_{\Psi}^{0}$, there exists $Q_{n} \in R, Q_{n} \geqq 0$ satisfying $\lim Q_{n} \Psi=\Phi$. By (3.13),

$$
\begin{gathered}
\left\|\Delta_{\Psi}^{\alpha}\left(Q_{n} \Psi-\Phi\right)\right\|^{2} \leqq\left\|\Delta_{\Psi}^{1 / 2}\left(Q_{n} \Psi-\Phi\right)\right\|^{2}+\left\|Q_{n} \Psi-\Phi\right\|^{2} \\
=\left\|J_{\Psi}\left(Q_{n} \Psi-\Phi\right)\right\|^{2}+\left\|Q_{n} \Psi-\Phi\right\|^{2} \rightarrow 0 .
\end{gathered}
$$

This proves $\Delta_{\Psi}^{\alpha} \mathscr{P}^{\sharp} \subset V_{\Psi}^{\alpha}$. By definition, $\Delta_{\Psi}^{\alpha} \mathscr{P}^{\sharp}$ contains a dense subset of $V_{\Psi}^{\alpha}$.

(4) This follows from $J_{\Psi}^{2}=1$ and

$$
J_{\Psi} \Delta_{\Psi}^{\alpha} Q \Psi=\Delta_{\Psi}^{1 / 2-\alpha} Q \Psi
$$

for $Q \in R, Q \geqq 0$.

(5) Let $Q_{1}, Q_{2} \in R, Q_{1} \geqq 0, Q_{2} \geqq 0$. Then

$$
\begin{aligned}
& \left(\Delta_{\Psi}^{\alpha} Q_{1} \Psi, \Delta_{\Psi}^{1 / 2-\alpha} Q_{2} \Psi\right)=\left(Q_{1} \Psi, \Delta_{\Psi}^{1 / 2} Q_{2} \Psi\right) \\
& \quad=\left(\Psi, Q_{1} j_{\psi}\left(Q_{2}\right) \Psi\right) \geqq 0
\end{aligned}
$$

due to $Q_{1} \geqq 0, j_{\Psi}\left(Q_{2}\right) \geqq 0$ and $\left[Q_{1}, j_{\Psi}\left(Q_{2}\right)\right]=0$. Hence

$$
\left(V_{\Psi}^{\alpha}\right)^{\prime} \supset V_{\Psi}^{1 / 2-\alpha}
$$

where $\left(V_{\Psi}^{\alpha}\right)^{\prime}$ denotes the set of all $\Phi$ such that $(\Phi, x) \geqq 0$ for every $x \in V_{\mathscr{w}}^{\alpha}$.

Next let $\Phi \in\left(\Delta_{\psi}^{\alpha} \mathscr{P}^{\sharp}\right)^{\prime}$. Let $f_{\beta}^{G}$ be given by (3.11) and let

$$
\Phi_{\beta} \equiv \int \Delta_{\Psi}^{i t} \Phi f_{\beta}^{G}(t) \mathrm{d} t
$$

Since $\Delta_{\Psi}^{\alpha} \cdot \mathscr{P}^{\sharp}$ is invariant under $\Delta_{\psi}^{i t}$, we have $\Phi_{\beta} \in\left(\Delta_{\Psi}^{\alpha} \cdot \mathscr{P}^{\sharp}\right)^{\prime}$. Furthermore,

$$
\Delta_{\Psi}^{i z} \Phi_{\beta}=\int \Delta_{\Psi}^{i t} \Phi f_{\beta}^{G}(t-z) \mathrm{d} t
$$

for real $z$ and the right hand side has an analytic continuation to all $z$. Hence $\Phi_{\beta}$ is an entire vector of $\log \Delta_{\psi}$ and is in domain of $\Delta_{\psi}^{2 z}$ for arbitrary $z$. Hence

$$
\Delta_{\psi}^{\alpha} \Phi_{\beta} \in\left(\mathscr{P}^{\sharp}\right)^{\prime}=\mathscr{P}^{b}=\Delta_{\Psi}^{1 / 2} V_{\Psi}^{0}
$$

where the last equality is due to (2) and (4), for example, and the

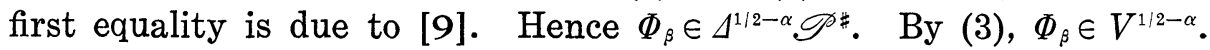
Since $\Phi=\lim _{\beta \rightarrow 0} \Phi_{\beta}$, we have $\Phi \in V_{\Psi^{\prime}}^{1 / 2-\alpha}$.

By (3), we now have

$$
\left(V_{\Psi}^{\alpha}\right)^{\prime} \subset\left(\Delta_{\Psi}^{\alpha} \mathscr{P}^{\sharp}\right)^{\prime} \subset V_{\Psi}^{1 / 2-\alpha} .
$$


By (4.6) and (4.9) we have (5).

(6) First consider the case $\alpha<1 / 4$.

For $\Phi \in V_{\psi}^{\alpha}$, there exists $Q_{n} \in R, Q_{n} \geqq 0$ such that $\Phi=\lim \Delta_{\psi}^{\alpha} Q_{n} \Psi$. We use (3.13), in which we replace $x$ by $\Delta_{\Psi}^{\alpha}\left(Q_{n}-Q_{m}\right) \Psi, Y$ by $\Delta_{\Psi}^{(1 / 2-2 \alpha)}$ and $\alpha$ by $1 / 2$. We have

$$
\begin{aligned}
& \left\|\Delta_{\Psi}^{(1 / 4-\alpha)}\left\{\Delta_{\Psi}^{\alpha}\left(Q_{n}-Q_{m}\right) \Psi\right\}\right\|^{2} \\
\leqq & \left\|\Delta_{\Psi}^{\alpha}\left(Q_{n}-Q_{m}\right) \Psi\right\|^{2}+\left\|\Delta_{\Psi}^{1 / 2-\alpha}\left(Q_{n}-Q_{m}\right) \Psi\right\|^{2} \\
= & \left\|\Delta_{\Psi}^{\alpha}\left(Q_{n}-Q_{m}\right) \Psi\right\|^{2}+\left\|J_{\Psi} \Delta_{\Psi}^{\alpha}\left(Q_{n}-Q_{m}\right) \Psi\right\|^{2} .
\end{aligned}
$$

Hence $\Delta_{\psi}^{1 / 4} Q_{n} \Psi$ is Cauchy and has a strong limit $\Delta_{\Psi}^{11 / 4-\alpha} \Phi$, which must be in $V_{\Psi}^{1 / 4}$ by definition. Hence

$$
V_{\Psi}^{\alpha} \subset \Delta_{\Psi}^{\alpha-1 / 4}\left\{V_{\Psi}^{1 / 4} \cap D\left(\Delta_{\Psi}^{\alpha-1 / 4}\right)\right\} .
$$

Let $x \in V_{\Psi}^{1 / 4} \cap D\left(\Delta_{\Psi}^{\alpha-1 / 4}\right)$ and $y \in V_{\Psi}^{0}$. Then

$$
\left(\Delta_{\Psi}^{1 / 2-\alpha} y, \Delta_{\Psi}^{\alpha-1 / 4} x\right)=\left(\Delta_{\Psi}^{1 / 4} y, x\right) \geqq 0
$$

due to $\Delta_{\Psi}^{1 / 4} y \in V_{\Psi}^{1 / 4} \subset\left(V_{\Psi}^{1 / 4}\right)^{\prime}$. By (3),

$$
\left(V_{\Psi}^{1 / 2-\alpha}\right)^{\prime} \supset \Delta_{\Psi}^{\alpha-1 / 4}\left\{V_{\Psi}^{1 / 4} \cap D\left(\Delta_{\Psi}^{\alpha-1 / 4}\right)\right\} .
$$

By (5), $\left(V_{\Psi^{1 / 2-\alpha}}^{1 /}\right)^{\prime}=V_{\Psi}^{\alpha}$ and hence we have (6).

The case $\alpha>1 / 4$ follows from the case $\alpha<1 / 4$ by (4).

(1) Let $\Phi \in V_{\psi}^{\alpha}$ and $-\Phi \in V_{\psi}^{\alpha} . \quad \mathrm{By}(5), \Phi \perp V_{\psi}^{1 / 2-\alpha}$. The linear span

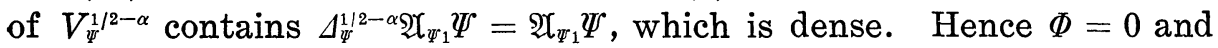
$V_{\Psi}^{\alpha}$ is pointed.

(7) If $Q \Psi \in V_{\Psi}^{\alpha}$, then $Q \Psi \in D\left(\Delta_{\Psi}^{1 / 2-2 \alpha}\right)$ and

$$
J_{\Psi} Q \Psi=\Delta_{\Psi}^{1 / 2} Q^{*} \Psi=\Delta_{\Psi}^{1 / 2-2 \alpha} Q \Psi
$$

due to (4.2). Hence $Q \Psi \in D\left(\Delta^{-2 \alpha}\right)$ and

$$
\Delta_{\Psi}^{-2 \alpha} Q \Psi=Q^{*} \Psi .
$$

By Lemma 6, we obtain the first half of (7) except for (4.4).

By (3) and (4), $V_{\psi^{1 / 2-\alpha}}^{{ }^{1 / 2}}{\Delta_{\psi}^{-\alpha}}_{\bar{P}^{b}}$. By (5),

$$
0 \leqq\left(\Delta_{\Psi}^{-\alpha} x, Q \Psi\right)=\left(x, \tau_{\Psi}(i \alpha) Q \Psi\right)
$$

for all $x \in \mathscr{P}^{b}$. Hence $\tau_{\Psi}(i \alpha) Q \geqq 0$ which shows (4.4).

Let $Q_{1}=\left(\Delta_{\psi}^{-\alpha} Q \Delta_{\psi}^{\alpha}\right)^{-}$. Then $Q \Delta_{\psi}^{\alpha} \Phi=\Delta_{\psi}^{\alpha} Q_{1} \Phi$ holds for a dense set of vectors $\Phi$. Hence $\Delta_{\psi}^{\alpha} Q^{*} \Psi=Q_{1}^{*} \Psi$, which implies $\Delta_{\Psi}^{1 / 2-\alpha} Q \Psi=J_{\psi} \Delta_{\psi}^{\alpha} Q^{*} \Psi=$ $\Delta_{\Psi}^{1 / 2} Q_{1} \Psi$. Therefore $Q \Psi=\Delta_{\Psi}^{\alpha} Q_{1} \Psi$. Since $Q_{1} \geqq 0$ by (4.4), $Q \Psi \in V_{\Psi}^{\alpha}$.

(8) If $\omega_{\Phi} \leqq l \omega_{\Psi}$, there exists $Q^{\prime} \in R^{\prime}$ such that $\omega_{\Phi}=\omega_{Q^{\prime} \Psi}$, and $\left\|Q^{\prime}\right\| \leqq l^{1 / 2}$. Then there exists a partial isometry $u^{\prime}$ in $R^{\prime}$ such that

$$
\Phi=u^{\prime} Q^{\prime} \Psi
$$


By (4.2) we have

$$
\Delta_{\Psi}^{1 / 2-2 \alpha} \Phi=J_{\Psi} \Phi=j_{\Psi}\left(u^{\prime} Q^{\prime}\right) \Psi .
$$

By (4), $J_{\Psi} \Phi \in V_{\Psi}^{1 / 2-\alpha}$ and hence by (7), $Q_{1}=j_{\Psi}\left(u^{\prime} Q^{\prime}\right) \in R$ has bounded $\tau_{\Psi}(z) Q_{1}$ for $\operatorname{Im} z \in[0,1-2 \alpha]$. Setting $Q=\tau_{\Psi}(i / 2-2 i \alpha) Q_{1}$, we have $\Phi=Q \Psi, Q \in R$ and $\|Q\| \leqq\left\|Q_{1}\right\| \leqq l^{1 / 2} . \quad\left(\Delta_{\psi}^{i z} Q \Delta_{\Psi}^{-i z}\right)^{-}=\tau_{\psi}\left(z^{\prime}\right) Q_{1}$ with $z^{\prime}=z+(1 / 2-2 \alpha) i$ and hence is bounded by $l^{1 / 2}$ for $\operatorname{Im} z \in[2 \alpha-1 / 2$, $1 / 2]$ and is positive for $\operatorname{Im} z=\alpha$.

(9) If $Q \Psi \in V_{\Psi}^{\alpha}, Q \in R$, then $\Delta_{\Psi}^{\alpha} Q \Delta_{\Psi}^{-\alpha}$ is bounded by $\|Q\|$, symmetric and affliated with $R$ due to (7). Hence

$$
\Delta_{\psi}^{\alpha}(\|Q\|-Q) \Delta_{\Psi}^{-\alpha}=\|Q\|-\Delta_{\psi}^{\alpha} Q \Delta_{\Psi}^{-\alpha}
$$

is bounded, positive and affiliated with $R$. By the last half of (7), $(\|Q\|-Q) \Psi \in V_{\psi}^{\alpha}$.

5. The cone $V_{\Psi}$. We denote $V_{\Psi}=V_{\psi}^{1 / 4}$ due to an importance of $V_{\Psi}^{1 / 4}$.

THEOREM 4. Let $\Psi$ be a cyclic and separating vector for $R$ on $H$.

(1) $V_{\Psi}$ is a pointed closed selfdual convex cone.

(2) $V_{\Psi}$ satisfies

$$
\begin{gathered}
\Delta_{\Psi}^{i t} V_{\Psi}=V_{\Psi}, \quad-\infty<t<\infty . \\
J_{\Psi} x=x, \quad x \in V_{\Psi} . \\
Q j_{\Psi}(Q) V_{\Psi} \subset V_{\Psi}, \quad Q \in R . \\
\left(x, Q j_{\Psi}(Q) y\right) \geqq 0, \quad x, y \in V_{\Psi}, \quad Q \in R .
\end{gathered}
$$

(3) $V_{\Psi}$ is the strong closure of the set of

$$
Q j_{\Psi}(Q) \Psi, \quad Q \in R .
$$

(4) If $\Phi \in V$ and $\Phi$ is separating or cyclic for $R$, then $\Phi$ is separating and cyclic for $R$ and $V_{\Phi}=V_{\Psi}$.

(5) If $\Phi$ is a cyclic and separating vector for $R$, then $\Phi \in V_{\Psi}$ if and only if $J_{\emptyset}=J_{\Psi}$ and

$$
(\Phi, z \Psi) \geqq 0
$$

for all $z \in R \cap R^{\prime}, z \geqq 0$.

(6) Any $\Phi \in H$ has a unique decomposition

$$
\Phi=\Phi_{1}-\Phi_{2}+i\left(\Phi_{3}-\Phi_{4}\right)
$$

such that $\Phi_{i} \in V_{\Psi}, i=1,2,3,4$, and

$$
\Phi_{1} \perp \Phi_{2}, \quad \Phi_{3} \perp \Phi_{4} .
$$


(7) If $\Phi_{1} \in V_{\Psi}, \Phi_{2} \in V_{\Psi}$ and $\Phi_{1} \perp \Phi_{2}$, then

$$
s^{R}\left(\Phi_{1}\right) \perp s^{R}\left(\Phi_{2}\right), \quad s^{R^{\prime}}\left(\Phi_{1}\right) \perp s^{R^{\prime}}\left(\Phi_{2}\right),
$$

where $s^{R}(\Phi)$ and $s^{R^{\prime}}(\Phi)$ denote projections onto closures of $R^{\prime} \Phi$ and $R \Phi$, respectively.

(8) If $\Phi_{1} \in V_{\Psi}$ and $\Phi_{2} \in V_{\Psi}$, then

$$
\left\|\omega_{\Phi_{1}}^{R}-\omega_{\Phi_{2}}^{R}\right\| \geqq\left\|\Phi_{1}-\Phi_{2}\right\|^{2}
$$

where $\omega_{\phi}^{R}$ is the expectation functional on $R$ by a vector $\Phi$.

Proof. (1), (5.1) and (5.2) follows from Theorem 3. Because

$$
\left\{Q j_{\Psi}(Q)\right\}\left\{Q_{1} j_{\Psi}\left(Q_{1}\right)\right\}=\left(Q Q_{1}\right) j_{\Psi}\left(Q Q_{1}\right),
$$

(5.3) follows from (3). (5.4) then follows by $V_{\psi}^{\prime}=V_{\psi}$.

(3) Let $Q\left(f_{\beta}^{G}\right)$ be given by (3.7) and (3.11) for $Q \in R$. Then

$$
\begin{aligned}
Q\left(f_{\beta}^{G}\right) j_{\Psi}\left(Q\left(f_{\beta}^{G}\right)\right) \Psi & =Q\left(f_{\beta}^{G}\right) \Delta_{\Psi}^{1 / 2} Q\left(f_{\beta}^{G}\right)^{*} \Psi \\
& =\Delta_{\Psi}^{1 / 4} Q_{1} Q_{1}^{*} \Psi \in V_{\Psi}
\end{aligned}
$$

where

$$
Q_{1}=\tau_{\psi}(i / 4) Q\left(f_{\beta}^{G}\right) \in R
$$

Hence

$$
Q j_{\Psi}(Q) \Psi=\lim _{\beta \rightarrow 0} Q\left(f_{\beta}^{G}\right) j_{\Psi}\left(Q\left(f_{\beta}^{G}\right)\right) \Psi \in V_{\Psi} .
$$

On the other hand, if we set

$$
Q_{2 \beta} \equiv \tau_{\Psi}(-i / 4)\left\{Q^{1 / 2}\left(f_{\beta}^{G}\right)\right\}, \quad Q \in R, \quad Q \geqq 0,
$$

then

$$
Q_{2 \beta} j_{\Psi}\left(Q_{2 \beta}\right) \Psi=Q_{2 \beta} \Delta_{\Psi}^{1 / 2} Q_{2 \beta}^{*} \Psi=\Delta_{\psi}^{1 / 4} Q^{1 / 2}\left(f_{\beta}^{G}\right)^{2} \Psi
$$

We have

$$
\begin{aligned}
& \lim _{\beta \rightarrow 0} Q^{1 / 2}\left(f_{\beta}^{G}\right)^{2} \Psi=\left(Q^{1 / 2}\right)^{2} \Psi=Q \Psi, \\
& \\
& \quad\left\|\Delta_{\Psi}^{1 / 4}\left\{Q^{1 / 2}\left(f_{\beta}^{G}\right)^{2} \Psi-Q \Psi\right\}\right\|^{2} \\
& \leqq\left\|\Delta_{\Psi}^{1 / 2}\left\{Q^{1 / 2}\left(f_{\beta}^{G}\right)^{2} \Psi-Q \Psi\right\}\right\|^{2}+\left\|Q^{1 / 2}\left(f_{\beta}^{G}\right)^{2} \Psi-Q \Psi\right\|^{2} \\
&= 2\left\|Q^{1 / 2}\left(f_{\beta}^{G}\right)^{2} \Psi-Q \Psi\right\|^{2} \rightarrow 0 .
\end{aligned}
$$

Hence $\Delta_{\Psi}^{1 / 4} \mathscr{P}^{\sharp}$ is in the strong closure of the set (5.5) and we have (3).

(4) If $R^{\prime} \Phi$ or $R \Phi$ is dense, then $R \Phi=J_{\Psi} R^{\prime} J_{\Psi} \Phi=J_{\Psi} R^{\prime} \Phi$ or $R^{\prime} \Phi=$ $J_{\Psi} R J_{\Psi} \Phi=J_{\Psi} R \Phi$ is dense. Hence if $\Phi$ in $V_{\Psi}$ is separating or cyclic, then $\Phi$ is cyclic and separating. If $\Phi \in V_{\Psi}$, then $J_{\Psi}$ satisfies 


$$
J_{\Psi} \Phi=\Phi, \quad\left(\Phi, Q j_{\Psi}(Q) \Phi\right) \geqq 0
$$

due to (5.2) and (5.4). Hence $J_{\Phi}=J_{\Psi}$ by Theorem 1. Since $V_{\Phi}$ is the strong closure of $Q j_{\phi}(Q) \Phi$, we have $V_{\phi} \subset V_{\Psi}$ due to (5.3) and $J_{\Psi}=J_{\Phi}$. Since $V_{\Phi}$ and $V_{\Psi}$ are selfdual, we have $V_{\Phi}=V_{\Phi}^{\prime} \supset V_{\Psi}^{\prime}=V_{\Psi}$ and hence $V_{\Phi}=V_{\Psi}$.

(5) If $\Phi \in V_{\Psi}$, then $J_{\Phi}=J_{\Psi}$ as we have seen and (5.6) holds because $z=z^{1 / 2} j_{\Psi}\left(z^{1 / 2}\right)$ due to Lemma 3. Conversely, assume $J_{\emptyset}=J_{\Psi}$. By (6) and (7), which we shall prove below, we have

$$
\begin{gathered}
\Phi=\Phi_{1}-\Phi_{2}, \quad \Phi_{1} \in V_{\Psi}, \quad \Phi_{2} \in V_{\Psi}, \\
s^{R}\left(\Phi_{1}\right) \perp s^{R}\left(\Phi_{2}\right) .
\end{gathered}
$$

Assume that $\left(\Phi_{1}, Q j_{y}(Q) \Phi_{2}\right)>0$ for some $Q \in R$. Let $Q_{1}=s^{R}\left(\Phi_{1}\right) Q s^{R}\left(\Phi_{2}\right)$. We then have by (5.12)

$$
\begin{aligned}
& \left(\Phi, Q_{1} j_{w}\left(Q_{1}\right) \Phi\right) \\
& \quad=-\left(\Phi_{1}, Q_{1} j_{\Psi}\left(Q_{1}\right) Q_{2}\right)=-\left(\Phi_{1}, Q j_{\Psi}(Q) \Phi_{2}\right)<0,
\end{aligned}
$$

where we have used $s^{R}\left(\Phi_{k}\right) \Phi_{k}=\Phi_{k}, j_{w}\left\{s^{R}\left(\Phi_{k}\right)\right\}=s^{R^{\prime}}\left(\Phi_{k}\right)$ (because of $\left.J_{\Psi} R^{\prime} \Phi_{k}=j_{\Psi}\left(R^{\prime}\right) J_{\Psi} \Phi_{k}=R \Phi_{k}\right)$ and $s^{R^{\prime}}\left(\Phi_{k}\right) \Phi_{k}=\Phi_{k}$, in the second equality. This contradicts with $J_{\Psi}=J_{\Phi}$ and (5.4) for the cone $V_{\Phi}$. Hence

$$
\left(\Phi_{1}, Q j_{\Psi}(Q) \Phi_{2}\right)=0
$$

due to (5.4) and (5.11).

From (5.13), we have

$$
s^{W^{\prime}}\left(\Phi_{1}\right) \perp s^{W^{\prime}}\left(\Phi_{2}\right)
$$

where $W$ is the von Neumann algebra generated by $Q j_{y}(Q)$. By Lemma $1, W^{\prime}=R \cap R^{\prime}$. Hence $z \equiv s^{W W^{\prime}}\left(\Phi_{2}\right) \in R \cap R^{\prime}$ and

$$
(\Psi, z \Phi)=-\left(\Psi, \Phi_{2}\right) \geqq 0
$$

by (5.6). Since $\Phi_{2} \in V_{\Psi}$, we have $\left(\Psi, \Phi_{2}\right) \geqq 0$ by $V_{\Psi}^{\prime}=V_{\Psi}$ and hence $\left(\Psi, \Phi_{2}\right)=0$. We shall see that this implies $\Phi_{2}=0$ in the proof of (7) and hence $\Phi=\Phi_{1} \in V_{\mathbb{T}}$.

(6) Let $\Phi \in H$. Define

$$
\Phi_{r}=2^{-1}\left(\Phi+J_{y} \Phi\right), \quad \Phi_{i}=(2 i)^{-1}\left(\Phi-J \Phi_{\Psi}\right) .
$$

Then

$$
\Phi=\Phi_{r}+i \Phi_{i}, \quad J_{y} \Phi_{r}=\Phi_{r}, \quad J_{q} \Phi_{i}=\Phi_{i} .
$$

Conversely, if (5.15) is satisfied, $\Phi_{r}$ and $\Phi_{i}$ are uniquely given by (5.14).

We now show that any $\Phi \in H$ satisfying $J_{\Psi} \Phi=\Phi$ has a unique 
decomposition

$$
\Phi=\Phi_{1}-\Phi_{2}, \quad \Phi_{1} \in V_{\Psi}, \quad \Phi_{2} \in V_{\Psi}, \quad \Phi_{1} \perp \Phi_{2} .
$$

Let

$$
\begin{gathered}
d=\inf \left\{\left\|\Phi-\Phi^{\prime}\right\| ; \Phi^{\prime} \in V_{\Psi}\right\} \\
\lim _{n}\left\|\Phi_{n}^{\prime}-\Phi\right\|=d, \quad \Phi_{n}^{\prime} \in V_{\Psi} .
\end{gathered}
$$

Since (5.18) implies that the sequence $\Phi_{n}^{\prime}$ is uniformly bounded, there exists a weakly converging subsequence $\Phi_{n(k)}^{\prime}$ :

$$
w-\lim _{k} \Phi_{n(k)}^{\prime}=\Phi_{1} \text {. }
$$

Then

$$
\left\|\Phi-\Phi_{1}\right\|^{2}=\left\|\Phi_{1}\right\|^{2}+d^{2}-\lim \left\|\Phi_{n(k)}^{\prime}\right\|^{2} .
$$

By (5.17) and $\left\|\Phi_{1}\right\|^{2} \leqq \lim \left\|\Phi_{n(k)}^{\prime}\right\|^{2}$, we have

$$
\left\|\Phi-\Phi_{1}\right\|^{2}=d^{2} \text {. }
$$

Let $\Phi_{2}=\Phi_{1}-\Phi$ and $x \in V_{\Psi}$. Then $\Phi_{1}+\lambda x \in V_{\Psi}$ for $\lambda \geqq 0$. We have from (5.17) and (5.19)

$$
\begin{aligned}
\left\|\Phi_{2}\right\|^{2}=d^{2} & \leqq\left\|\Phi-\left(\Phi_{1}+\lambda x\right)\right\|^{2} \\
& =\left\|\Phi_{2}\right\|^{2}+\lambda\left\{2\left(\Phi_{2}, x\right)+\|x\|^{2} \lambda\right\}
\end{aligned}
$$

where $\left(\Phi_{2}, x\right)$ is real due to $J_{y} \Phi_{2}=\Phi_{2}$ and $J_{y} x=x$. We then have

$$
\left(\Phi_{2}, x\right) \geqq 0
$$

which implies $\Phi_{2} \in V_{\Psi}^{\prime}=V_{\Psi}$.

Since $\Phi_{1}$ and $\Phi_{2}$ are in $V_{\Psi},\left(\Phi_{1}, \Phi_{2}\right) \geqq 0$. For $\lambda>0$,

$$
d^{2} \leqq\left\|\Phi-(1-\lambda) \Phi_{1}\right\|^{2}=\left\|\Phi_{2}\right\|^{2}-\lambda\left(2\left(\Phi_{1}, \Phi_{2}\right)-\lambda\left\|\Phi_{1}\right\|^{2}\right)
$$

which implies $\left(\Phi_{1}, \Phi_{2}\right)=0$.

To prove the uniqueness of the decomposition (5.16), let $\Phi=\Phi_{1}$ $\Phi_{2}=\Phi_{1}^{\prime}-\Phi_{2}^{\prime}$ be two such decompositions. For any vectors $x_{1}, x_{2}, x_{3}$, we have

$$
G\left(x_{1}, x_{2}, x_{3}\right) \equiv \operatorname{det}\left(\left(x_{i}, x_{j}\right)\right)\left(=\operatorname{dex} X^{*} X\right) \geqq 0 .
$$

Since $\left(\Phi_{k}, \Phi_{l}^{\prime}\right)$ are all real, we have

$$
\begin{aligned}
0 \leqq & G\left(\Phi, \Phi_{1}^{\prime},-\Phi_{2}\right) \\
= & \left(\left\|\Phi_{1}\right\|^{2}-\left\|\Phi_{1}^{\prime}\right\|^{2}\right)\left\|\Phi_{1}^{\prime}\right\|^{2}\left\|\Phi_{2}\right\|^{2} \\
& -\left(\Phi_{1}^{\prime}, \Phi_{2}\right)^{2}\|\Phi\|^{2}-2\left\|\Phi_{1}^{\prime}\right\|^{2}\left\|\Phi_{2}\right\|^{2}\left(\Phi_{1}^{\prime}, \Phi_{2}\right),
\end{aligned}
$$




$$
\begin{aligned}
0 \leqq & G\left(\Phi, \Phi_{1},-\Phi_{2}^{\prime}\right) \\
= & \left(\left\|\Phi_{1}^{\prime}\right\|^{2}-\left\|\Phi_{1}\right\|^{2}\right)\left\|\Phi_{1}\right\|^{2}\left\|\Phi_{2}^{\prime}\right\|^{2} \\
& -\left(\Phi_{1}, \Phi_{2}^{\prime}\right)^{2}\|\Phi\|^{2}-2\left\|\Phi_{1}\right\|^{2}\left\|\Phi_{2}^{\prime}\right\|^{2}\left(\Phi_{1}, \Phi_{2}^{\prime}\right) .
\end{aligned}
$$

Since $\left(\Phi_{k}, \Phi_{l}^{\prime}\right) \geqq 0$ by $V_{\Psi}^{\prime}=V_{\Psi}$, either all terms in (5.21) are negative or all terms in (5.22) are negative. In the first case, all terms in (5.21) vanish and we have the following three alternatives:

Case (i). $\Phi_{1}^{\prime}=0, \Phi=-\Phi_{2}^{\prime}$. Then

$$
\left\|\Phi_{1}\right\|^{2}=\left(\Phi_{1}, \Phi\right)=-\left(\Phi_{1}, \Phi_{2}^{\prime}\right) \leqq 0
$$

and hence $\Phi_{1}=0=\Phi_{1}^{\prime}$ and $\Phi_{2}=-\Phi=\Phi_{2}^{\prime}$.

Case (ii). $\quad \Phi_{2}=0, \Phi=\Phi_{1}$. Then

$$
\left\|\Phi_{2}^{\prime}\right\|^{2}=-\left(\Phi_{2}^{\prime}, \Phi\right)=-\left(\Phi_{2}^{\prime}, \Phi_{1}\right) \leqq 0
$$

and hence $\Phi_{2}^{\prime}=0=\Phi_{2}, \Phi_{1}^{\prime}=\Phi=\Phi_{1}$.

Case (iii). $\quad\left(\Phi_{1}^{\prime}, \Phi_{2}\right)=0$ and $\left\|\Phi_{1}\right\|^{2}=\left\|\Phi_{1}^{\prime}\right\|^{2}$. Then

$$
\left\|\Phi_{1}\right\|^{2}=\left\|\Phi_{1}^{\prime}\right\|^{2}=\left(\Phi_{1}^{\prime}, \Phi\right)=\left(\Phi_{1}^{\prime}, \Phi_{1}\right)
$$

which implies $\left\|\Phi_{1}-\Phi_{1}^{\prime}\right\|^{2}=0$. Hence $\Phi_{1}=\Phi_{1}^{\prime}, \Phi_{2}=\Phi_{2}^{\prime}$. If all terms in (5.22) vanish, we have the same argument.

(7) First we prove that any nonzero $\Phi \in V_{\Psi}$ is never orthogonal to $\Psi$. By (3), there exists $Q_{n} \in R$ such that

$$
\Phi=\lim _{n} Q_{n} j_{\Psi}\left(Q_{n}\right) \Psi \text {. }
$$

Assume that $(\Psi, \Phi)=0$. Then

$$
\begin{aligned}
0 & =\lim _{n}\left(\Psi, Q_{n} j_{\psi}\left(Q_{n}\right) \Psi\right) \\
& =\lim _{n}\left\|\Delta_{\Psi}^{1 / 4} Q_{n} \Psi\right\|^{2} .
\end{aligned}
$$

Let $x=Q j(Q) \Psi, Q^{\prime} \in R, Q=Q^{\prime}\left(f_{\beta}^{G}\right)$. Then

$$
\begin{aligned}
(x, \Phi) & =\lim \left(x, Q_{n} j_{\Psi}\left(Q_{n}\right) \Psi\right) \\
& =\lim \left(j_{\Psi}\left(Q_{n}^{*} Q\right) \Psi, Q^{*} Q_{n} \Psi\right) \\
& =\lim \left\|\Delta_{\Psi}^{1 / 4} Q^{*} Q_{n} \Psi\right\|^{2} \\
& =\lim \left\|\left\{\tau_{\Psi}(i / 4) Q\right\}^{*} \Delta_{\Psi}^{1 / 4} Q_{n} \Psi\right\|^{2} \\
& =0 .
\end{aligned}
$$

By (3.12) and Lemma 1 (or (3) and (6)), such $x$ is total in $H$ and hence $\Phi=0$.

Since $V_{\Phi_{1}}=V_{\Psi}$ for any separating $\Phi_{1}$ in $V_{\Psi}$, we have

$$
\left(\Phi_{1}, \Phi_{2}\right)>0
$$

if $\Phi_{1} \in V_{\Psi}, \Phi_{2} \in V_{\Psi}, \Phi_{1}$ is separating for $R$ and $\Phi_{2} \neq 0$. 
We now assume that $\Phi_{1} \in V_{\Psi}, \Phi_{2} \in V_{\Psi}$ and $\Phi_{1} \perp \Phi_{2}$. Let $s$ and $s^{\prime}$ denote $s^{R}\left(\Phi_{1}\right)$ and $s^{R \prime}\left(\Phi_{1}\right)$, respectively. Since $J_{\Psi} R^{\prime} \Phi_{1}=j_{\Psi}\left(R^{\prime}\right) \Phi_{1}=R \Phi_{1}$, we have $j_{\Psi}(s)=s^{\prime}$. Hence $J_{\Psi}$ commutes with $s s^{\prime}$.

Consider the space $\hat{H}=s s^{\prime} H$ and a von Neumann algebra $\hat{R}=s R s s^{\prime}$ on $\hat{H} . \quad \Phi_{1}$ is in $\hat{H}$ and is cyclic and separating for $\hat{R}$ by definition of $s$ and $s^{\prime}$. Since $J_{\Psi}$ commutes with $s s^{\prime}$, the restriction of $J_{\Psi}$ to $\hat{H}$ is the modular conjugation operator $\hat{J}_{\Phi_{1}}$ for $\Phi_{1}$ on $\hat{H}$ due to Theorem 1 . We also have

$$
s s^{\prime} Q j_{\Psi}(Q) \Phi_{1}=s s^{\prime} Q j_{\Psi}(Q) s s^{\prime} \Phi_{1}=\hat{Q} j_{\Phi_{1}}(\widehat{Q}) \Phi_{1}
$$

where $\hat{Q}=s Q s$. Hence $s s^{\prime} V_{\Psi}=\hat{V}_{\Phi_{1}}$.

Let $\widehat{\Phi}_{2}=s s^{\prime} \Phi_{2} . \quad \hat{\Phi}_{2} \in \hat{V}_{\Phi_{1}}$ because $\Phi_{2} \in V_{y}$. We also have

$$
\left(\widehat{\Phi}_{2}, \Phi_{1}\right)=\left(\Phi_{2}, \Phi_{1}\right)=0 .
$$

By (5.23), we have $\widehat{\Phi}_{2}=0$.

Denoting $\varphi=(1-s)\left(1-s^{\prime}\right) \Phi_{2}, \varphi_{1}=s\left(1-s^{\prime}\right) \Phi_{2}$, and $\varphi_{2}=(1-s) s^{\prime} \Phi_{2}$, we have

$$
\Phi_{2}=\varphi+\varphi_{1}+\varphi_{2} .
$$

Since $J_{\Psi} \Phi_{2}=\Phi_{2}$, and $j_{Y}(s)=s^{\prime}$, we have $J_{\Psi} \varphi_{1}=\varphi_{2}$. We now prove $\varphi_{1}=\varphi_{2}=0$.

Assume $\varphi_{1} \neq 0$ and let $s_{k}=s^{R}\left(\varphi_{k}\right), s_{k}^{\prime}=s^{R^{\prime}}\left(\varphi_{k}\right), k=1,2$. Then $j_{\Psi}\left(s_{1}\right)=s_{2}^{\prime}, j_{\Psi}\left(s_{2}\right)=s_{1}^{\prime}, s_{1} \leqq s, s_{2} \leqq 1-s$. Let $c(E)$ denote the central support of $E \in\left(R \cup R^{\prime}\right)^{\prime \prime}$. Then $j_{Y}(c(E))=c(E)^{*}=c(E)$ by Lemma 3 . Hence $c\left(j_{y}(E)\right)=c(E)$. Setting $E=s_{1} s_{1}^{\prime}$, we have $c\left(s_{1} s_{1}^{\prime}\right)=c\left(s_{2} s_{2}^{\prime}\right)$. Since $s_{1} s_{1}^{\prime} \varphi_{1}=\varphi_{1} \neq 0, c\left(s_{1} s_{1}^{\prime}\right) \neq 0$. We have $c\left(s_{1}\right) \geqq c\left(s_{1} s_{1}^{\prime}\right)=c\left(s_{2} s_{2}^{\prime}\right)$ and $c\left(s_{2}\right) \geqq$ $c\left(s_{2} s_{2}^{\prime}\right)$. Therefore, there exists a partial isometry $u \in R$ such that $u^{*} u \leqq s_{1}, u u^{*} \leqq s_{2}, c\left(u u^{*}\right)=c\left(u^{*} u\right)=c\left(s_{2} s_{2}^{\prime}\right)$.

Since $s_{1}$ is the support of $\varphi_{1}, u^{*} u \varphi_{1} \neq 0$. Then $s^{\prime \prime} \equiv s^{R \prime}\left(u^{*} u \varphi_{1}\right) \leqq s_{1}^{\prime}$ is nonzero and $c\left(s^{\prime \prime}\right) \leqq c\left(s_{2} s_{2}^{\prime}\right) \leqq c\left(s_{2}^{\prime}\right)$. Hence there exists a partial isometry $v \in R^{\prime}$ such that $v^{*} v \leqq s^{\prime \prime}, v v^{*} \leqq s_{2}^{\prime}, v \neq 0$. Again $v^{*} v u^{*} u \varphi_{1} \neq 0$.

Since

$$
u v \varphi_{1} \in u H \cong s_{2} H, \quad u v \varphi_{1} \in v H \subseteq s_{2}^{\prime} H,
$$

there exists $A \in s_{2} R s_{2}$ such that

$$
\operatorname{Re}\left(u v \varphi_{1}, A \varphi_{2}\right)>0 \text {. }
$$

Let $Q=A^{*} u-j_{y}(v) . \quad \mathrm{A}^{*} u$ vanishes on $(1-s) H$ and its range is in $(1-s) H . \quad j_{\Psi}(v)$ vanishes on $s H$ and its range is in $s H . \quad v$ vanishes on $s^{\prime} H$ and its range is in $s^{\prime} H . \quad j_{Y}\left(A^{*} u\right)$ vanishes on $\left(1-s^{\prime}\right) H$ and its range is in $\left(1-s^{\prime}\right) H$. Therefore, 


$$
\begin{aligned}
0 & \leqq \\
& =-\left(\Phi_{2}, Q j_{\Psi}(Q) \Phi_{2}\right) \\
& =-2 \operatorname{Re}\left(\varphi_{2}, A^{*} u v j_{\Psi}(v) \varphi_{2}\right)-\left(\Phi_{2}, A^{*} u v \varphi_{1}\right)
\end{aligned}
$$

where we have used $J_{\Psi} \varphi_{1}=\varphi_{2}, \varphi_{1}=J_{\Psi} \varphi_{2}$. This contradicts with (5.24). Therefore $\varphi_{1}=\varphi_{2}=0$ and $\Phi_{2}=\varphi$.

We now have

$$
\begin{gathered}
s^{R}\left(\Phi_{2}\right)=s^{R}(\varphi) \leqq 1-s, \\
s^{R^{\prime}}\left(\Phi_{2}\right)=s^{R^{\prime}}(\varphi) \leqq 1-s^{\prime} .
\end{gathered}
$$

Hence (5.9) is satisfied.

(8) For $\Phi_{1} \in V_{\Psi}$ and $\Phi_{2} \in V_{\Psi}$, we have a decomposition

$$
\Phi_{1}-\Phi_{2}=\Phi_{+}-\Phi_{-}
$$

satisfying $\Phi_{ \pm} \in V_{\Psi}, \Phi_{+} \perp \Phi_{-}$, due to (6). By (7), we have $s^{R}\left(\Phi_{+}\right) \perp s^{R}\left(\Phi_{-}\right)$.

Let $E \equiv s^{R}\left(\Phi_{+}\right)-s^{R}\left(\Phi_{-}\right)$. Then $\|E\| \leqq 1$. We have

$$
\begin{aligned}
& \left\|\omega_{\Phi_{1}}-\omega_{\Phi_{2}}\right\| \geqq\left\|\omega_{\Phi_{1}}(E)-\omega_{\Phi_{2}}(E)\right\| \\
& \quad=2^{-1}\left|\left(\Phi_{1}-\Phi_{2}, E\left(\Phi_{1}+\Phi_{2}\right)\right)+\left(\Phi_{1}+\Phi_{2}, E\left(\Phi_{1}-\Phi_{2}\right)\right)\right| \\
& \quad=\left|\operatorname{Re}\left(\Phi_{+}+\Phi_{-}, \Phi_{1}+\Phi_{2}\right)\right| \\
& \quad=\left(\Phi_{+}+\Phi_{-}, \Phi_{1}+\Phi_{2}\right) \\
& \quad \geqq\left(\Phi_{+}-\Phi_{-}, \Phi_{1}-\Phi_{2}\right)=\left\|\Phi_{1}-\Phi_{2}\right\|^{2},
\end{aligned}
$$

where we have used $\left(\Phi_{1}, \Phi_{-}\right) \geqq 0$ and $\left(\Phi_{2}, \Phi_{+}\right) \geqq 0$ due to $\Phi_{1}, \Phi_{2}, \Phi_{-}, \Phi_{+} \in V_{\Phi}$.

\section{Some Radon-Nikodym theorems.}

THEOREM 5. Let $\mu$ be a normal positive linear functional on a von Neumann algebra $R$ with a cyclic and separating vector $\Psi$ such that $\mu \leqq \omega_{\Psi}$. Then there exists $h_{\alpha} \in R,\left\|h_{\alpha}\right\| \leqq 1, h_{\alpha} \geqq 0$ for each $\alpha \in[0,1]$ such that

$$
2 \mu(Q)=\left(\Delta_{\Psi}^{\alpha / 2} Q^{*} \Psi, \Delta_{\Psi}^{\alpha / 2} h_{\alpha} \Psi\right)+\left(\Delta_{\Psi}^{\alpha / 2} h_{\alpha} \Psi, \Delta_{\Psi}^{\alpha / 2} Q \Psi\right)
$$

Proof. Let $h \in R, h^{*}=h$ and

$$
f_{h}^{\alpha}(Q)=\left\{\left(\Delta_{\Psi}^{\alpha / 2} Q^{*} \Psi, \Delta_{\Psi}^{\alpha / 2} h \Psi\right)+\left(\Delta_{\Psi}^{\alpha / 2} h \Psi, \Delta_{\Psi}^{\alpha / 2} Q \Psi\right)\right\} / 2 .
$$

If $\alpha \leqq 1 / 2$, then

$$
f_{h}^{\alpha}(Q)=(1 / 2)\left\{\left(\Psi, Q \Delta_{\Psi}^{\alpha} h \Psi\right)+\left(\Delta_{\Psi}^{\alpha} h \Psi, Q \Psi\right)\right\} .
$$

If $\alpha \geqq 1 / 2$, then

$$
\begin{aligned}
f_{h}^{\alpha}(Q)= & (1 / 2)\left\{\left(J_{\Psi} \Delta_{\Psi}^{\alpha / 2} h \Psi, J_{\Psi} \Delta_{\Psi}^{\alpha / 2} Q^{*} \Psi\right)\right. \\
& \left.+\left(J_{\Psi} \Delta_{\Psi}^{\alpha / 2} Q \Psi, J_{\Psi} \Delta_{\Psi}^{\alpha} / 2 h \Psi\right)\right\}=f_{h}^{1-\alpha}(Q) .
\end{aligned}
$$


Hence $f_{h}^{\alpha}$ is a normal linear functional on $R$. If $Q^{*}=Q$, then

$$
f_{h}^{\alpha}(Q)=\operatorname{Re}\left(\Delta_{\Psi}^{a / 2} Q^{*} \Psi, \Delta_{\Psi}^{\alpha / 2} h \Psi\right)
$$

and hence $f_{h}^{\alpha}$ is selfadjoint. Since

$$
f_{h}^{\alpha}(Q)=\left\{\left(\Delta_{\psi}^{\alpha} Q^{*} \Psi, h \Psi\right)+\left(h \Psi, \Delta_{\Psi}^{\alpha} Q \Psi\right)\right\} / 2
$$

for $\alpha \leqq 1 / 2$ and $f_{h}^{\alpha}(Q)=f_{h}^{1-\alpha}(Q), f_{h}^{\alpha}$ is weakly continuous in $h$.

Let $F$ be the set of $f_{h}^{\alpha}, h \in R, h^{*}=h, 1 \geqq h \geqq 0$. Then as an image of a compact, convex set under continuous real linear map, $F$ is weakly compact and convex. $F$ contains 0 . Let $F^{0}$ be the polar of $F$, namely the set of $Q \in R, Q^{*}=Q$ and $f(Q) \leqq 1$ for all $f \in F$. Then $\left(F^{0}\right)^{0}=F$, where $\left(F^{0}\right)^{0}$ is the set of all normal linear selfadjoint functionals $f$ satisfying $f(Q) \leqq 1$ for all $Q \in F^{0}$.

For each real $\alpha \in[0,1]$, consider

$$
\begin{gathered}
m_{h}^{\alpha}(Q)=\sup _{t} \operatorname{Re} f_{h}(\alpha+i t), \\
f_{h}(\alpha+i t)=\left(\Delta_{\Psi}^{(\alpha-i t) / 2} Q^{*} \Psi, \Delta_{\Psi}^{(\alpha+i t) / 2} h \Psi\right) .
\end{gathered}
$$

$f_{h}(z)$ is obviously an analytic function of $z$ for $\operatorname{Re} z \in(0,1)$. It is continuous for $\operatorname{Re} z \in[0,1]$. Furthermore,

$$
\begin{aligned}
& \left|f_{h}(\alpha+i t)\right| \leqq\left\|\Delta_{\Psi}^{(\alpha-i t) / 2} Q^{*} \Psi\right\|\left\|\Delta_{\Psi}^{(\alpha+i t) / 2} h \Psi\right\| \\
& \quad \leqq\left\{\left\|J_{\Psi} Q \Psi\right\|^{2}+\left\|Q^{*} \Psi\right\|^{2}\right\}^{1 / 2}\left\{\left\|J_{\Psi} h \Psi\right\|^{2}+\|h \Psi\|^{2}\right\}^{1 / 2} .
\end{aligned}
$$

By the three line theorem,

$$
\sup _{t} \operatorname{Re} f_{h}(\alpha+i t)=\log \sup _{t}\left|e^{f_{h}^{(\alpha+i t)}}\right|
$$

is a convex function of $\alpha$. Hence

$$
g^{\alpha}(Q)=\sup _{h}\left\{m_{h}^{\alpha}(Q) ; h \in R, h^{*}=h, 1 \geqq h \geqq 0\right\}
$$

is also a convex function of $\alpha$.

Since $f_{h}(\alpha+i t)=f_{h^{\prime}}(\alpha), h^{\prime}=\Delta_{\psi}^{i t} h \Delta_{\psi}^{-i t}$, we have for $Q^{*}=Q$

$$
g^{\alpha}(Q)=\sup _{h}\left\{f_{h}^{\alpha}(Q) ; h \in R, h^{*}=h, 1 \geqq h \geqq 0\right\} .
$$

By (6.4) we have

$$
g^{\alpha}(Q)=g^{1-\alpha}(Q)
$$

Due to convexity,

$$
g^{\alpha}(Q) \geqq g^{1 / 2}(Q)
$$

We have

$$
\begin{aligned}
f_{h}^{1 / 2}(Q) & =\left(\Psi, j_{\Psi}(h) Q \Psi\right) \\
& =\omega_{\varphi}(Q)
\end{aligned}
$$




$$
\Phi=j_{\Psi}(h)^{1 / 2} \Psi .
$$

The set of such $\omega_{\Phi}$ for $h \in R, h^{*}=h, 1 \geqq h \geqq 0$ is exactly the set of all normal positive linear functionals $\mu$ of $R$ satisfying $\mu \leqq \omega_{\psi}$. Hence $g^{1 / 2}(Q) \geqq \mu(Q)$ and by $(6.6)$

$$
g^{\alpha}(Q) \geqq \mu(Q)
$$

for any $Q^{*}=Q, Q \in R, \alpha \in[0,1]$. Hence $\mu \in\left(F^{0}\right)^{0}=F$.

REMARK. $h_{1-\alpha}=h_{\alpha} . \quad h_{\alpha}$ is unique. (If $\mu=0$, set $Q=h_{\alpha}$ )

CoRollary. If $\Phi \in V_{\Psi}^{\alpha}, l \Psi-\Phi \in V_{\Psi}^{\alpha}$ and $\alpha \leqq 1 / 4$, then there exists $h \in R$ such that $0 \leqq h \leqq l$ and

$$
2 \Phi=h \Psi+\Delta_{\Psi}^{2 \alpha} h \Psi .
$$

Such $h$ is unique. If $\Phi \in V_{\Psi}^{\alpha}, l \Psi-\Phi \in V_{\Psi}^{\alpha}$ and $\alpha \geqq 1 / 4$, then there exists $h^{\prime} \in R^{\prime}$ such that $0 \leqq h^{\prime} \leqq l$ and

$$
2 \Phi=h^{\prime} \Psi+\Delta_{\Psi}^{2 \alpha-1} h^{\prime} \Psi .
$$

Such $h^{\prime}$ is unique.

Proof. Let $\alpha \leqq 1 / 4, \beta=1 / 2-\alpha$ and

$$
\mu(Q) \equiv\left(\Phi, \Delta_{\Psi}^{\beta} Q \Psi\right) / l, \quad Q \in R .
$$

Since $\Delta_{\psi}^{3} Q \Psi \in V_{\Psi}^{\beta}=\left(V_{\Psi}^{\alpha}\right)^{\prime}$ for $Q \geqq 0$, we have $\mu \geqq 0$. By $l \Psi-\Phi \in V_{\Psi}^{\alpha}$, we also have $\mu \leqq \omega_{\Psi}$. By applying Theorem 5 to $\mu$ and setting $h=l h_{\beta}$, we have

$$
2 l \mu(Q)=\left(h \Psi, \Delta_{\Psi}^{\beta} Q \Psi\right)+\left(\Delta_{\Psi}^{8} Q^{*} \Psi, h \Psi\right)
$$

Since

$$
\begin{aligned}
\left(\Delta_{\Psi}^{\beta} Q^{*} \Psi, h \Psi\right) & =\left(J_{\Psi} h \Psi, J_{\Psi} \Delta_{\Psi}^{\beta} Q^{*} \Psi\right) \\
& =\left(\Delta_{\Psi}^{1 / 2} h \Psi, \Delta_{\Psi}^{1 / 2-\beta} Q \Psi\right) \\
& =\left(\Delta_{\Psi}^{2 \alpha} h \Psi, \Delta_{\Psi}^{\beta} Q \Psi\right),
\end{aligned}
$$

we have (6.7).

If $h_{1}$ and $h_{2}$ yield the same $\Phi$, then we have for $h=h_{1}-h_{2}$

$$
0=\left(h \Psi+\Delta_{\Psi}^{2 \alpha} h \Psi, h \Psi\right)=\|h \Psi\|^{2}+\left\|\Delta_{\Psi}^{\alpha} h \Psi\right\|^{2} .
$$

Hence $h \Psi=0$ and $h_{1}=h_{2}$, which proves the uniqueness of $h$.

If $\alpha \geqq 1 / 4$, then we interchange the role of $R$ and $R^{\prime}$. Then $A_{\Psi}^{-1}$ replaces $\Delta_{T}$ and $1 / 2-\alpha$ replaces $\alpha$. We then obtain the latter half of corollary. 
REMARK. If $\alpha=1 / 4$, then $\Delta_{\Psi}^{2 \alpha} h \Psi=J_{\Psi} h \Psi, \Delta_{\Psi}^{2 \alpha-1} h^{\prime} \Psi=J_{\Psi} h^{\prime} \Psi$ and hence $h^{\prime}=j_{w}(h)$.

THEOREM 6. For any normal state $\mu$ of a von Neumann algebra $R$ with a cyclic and separating vector $\Psi$, there exists $\Phi \in V_{\Psi}$ such that $\omega_{\Phi}=\mu$.

We first prove a technical lemma.

LEMma 7. Let $\Psi$ be a cyclic and separating vector for $R$ and $S$ be an operator in $R$ with a bounded inverse $S^{-1} \in R$ such that $S \Psi \in V_{\Psi} . \quad$ If $\Delta_{\Psi}^{1 / 2} Q \Psi=Q_{1} \Psi$ for some $Q \in R$ and $Q_{1} \in R$, then

$$
\Delta_{S \Psi}^{1 / 2} Q(S \Psi)=Q_{2}(S \Psi), \quad Q_{2}=S Q_{1} S^{-1} .
$$

Proof. By using $J_{\Psi}=J_{S \Psi}$ due to $S \Psi \in V_{\Psi}$, we have

$$
\begin{aligned}
\Delta_{S \Psi}^{1 / 2} Q S \Psi & =J_{S \Psi} Q^{*} S \Psi=j_{S \Psi}\left(Q^{*}\right) S \Psi \\
& =S j_{S \Psi}\left(Q^{*}\right) \Psi=S j_{\Psi}\left(Q^{*}\right) \Psi \\
& =S J_{\Psi} Q^{*} \Psi=S \Delta_{\Psi}^{1 / 2} Q \Psi \\
& =S Q_{1} \Psi=S Q_{1} S^{-1}(S \Psi) .
\end{aligned}
$$

Proof of Theorem 6.

Step (i). Let $0<\delta \leqq 2^{-4}$. We prove that if $\Psi_{1}$ is cyclic and separating vector belonging to $V_{\Psi}, t_{1} \in R, t_{1}^{\prime} \in R$ and

$$
\begin{gathered}
\Phi_{1}=\Psi_{1}+t_{1} \Psi_{1}, \\
\left\|t_{1}\right\| \leqq \delta, \quad\left\|t_{1}^{\prime}\right\| \leqq \delta, \\
\Delta_{\Psi_{1}^{1}}^{1 / 2} t_{1} * \Psi_{1}=t_{1}^{\prime} \Psi_{1},
\end{gathered}
$$

then there exists $\Phi \in V_{\Psi}$ such that

$$
\omega_{\Phi}=\omega_{\Phi_{1}} .
$$

We first note that by Theorem 4 (4) and (5), $J_{\Psi_{1}}=J_{\Psi}$ and $V_{\Psi}=V_{\Psi_{1}}$. Let

$$
t_{1 \pm} \equiv(1 / 2)\left\{t_{1} \pm t_{1}^{\prime}\right\}
$$

Then

$$
J_{\Psi_{1}} t_{1 \pm} \Psi_{1}= \pm t_{1 \pm} \Psi_{1}
$$

By Theorem 4 (6) and (7), there exists $\Psi_{11} \in V_{\Psi_{1}}$ and $\Psi_{12} \in V_{\Psi_{1}}$ such that 


$$
\begin{gathered}
-i t_{1-} \Psi_{1}=\Psi_{11}-\Psi_{12}, \\
s^{R}\left(\Psi_{11}\right) \perp s^{R}\left(\Psi_{12}\right) .
\end{gathered}
$$

Let

$$
t_{11} \equiv-i s^{R}\left(\Psi_{11}\right) t_{1-}, \quad t_{12} \equiv i s^{R}\left(\Psi_{12}\right) t_{1-}
$$

Then

$$
\begin{array}{cc}
t_{11} \Psi_{1}=\Psi_{11} \in V_{\Psi_{1}}, & t_{12} \Psi_{1}=\Psi_{12} \in V_{\Psi_{1}}, \\
\left\|t_{11}\right\| \leqq \delta, & \left\|t_{12}\right\| \leqq \delta .
\end{array}
$$

By Theorem 3 (9), $\left(\delta-t_{11}\right) \Psi_{1} \in V_{\Psi_{1}},\left(\delta-t_{12}\right) \Psi_{1} \in V_{\Psi_{1}}$. Hence by corollary to Theorem 5 , there exists $h_{1} \in R$ and $h_{2} \in R$ such that

$$
\begin{gathered}
0 \leqq h_{1} \leqq \delta, \quad 0 \leqq h_{2} \leqq \delta, \\
t_{11} \Psi_{1}=\left(h_{1} \Psi_{1}+J_{\Psi_{1}} h_{1} \Psi_{1}\right) / 2, \\
t_{12} \Psi_{1}=\left(h_{2} \Psi_{1}+J_{\Psi_{1}} h_{2} \Psi_{1}\right) / 2 .
\end{gathered}
$$

From $J_{\Psi_{1}} h_{k} \Psi_{1}=\Delta_{\Psi_{1}}^{1 / 2} h_{k}^{*} \Psi_{1}=\Delta_{\Psi_{1}}^{1 / 2} h_{k} \Psi_{1}$, we obtain

$$
\begin{aligned}
& h_{1}^{\prime} \equiv \tau_{\Psi_{1}}(-i / 2) h_{1}=2 t_{11}-h_{1}, \\
& h_{2}^{\prime} \equiv \tau_{\Psi_{1}}(-i / 2) h_{2}=2 t_{12}-h_{2} .
\end{aligned}
$$

Thus

$$
\left\|h_{1}^{\prime}-h_{2}^{\prime}\right\| \leqq 2\left\|t_{1-}\right\|+\left\|h_{1}-h_{2}\right\| \leqq 3 \delta
$$

We set

$$
\begin{gathered}
\Phi_{2} \equiv u^{\prime} \Phi_{1}, \quad u^{\prime} \equiv \exp \left\{-i j_{\Psi_{1}}\left(h_{1}-h_{2}\right)\right\}, \\
\Psi_{2} \equiv S_{1} \Psi_{1}, \quad S_{1} \equiv 1+t_{1}-i\left(h_{1}^{\prime}-h_{2}^{\prime}\right), \\
t_{2}^{\prime} \equiv\left(1+t_{1}\right)\left(-1+i\left(h_{1}^{\prime}-h_{2}^{\prime}\right)+\exp \left\{-i\left(h_{1}^{\prime}-h_{2}^{\prime}\right)\right\}\right)-i t_{1}\left(h_{1}^{\prime}-h_{2}^{\prime}\right), \\
t_{2} \equiv t_{2}^{\prime} S_{1}^{-1} .
\end{gathered}
$$

Since $u^{\prime}$ commutes with $t_{1}$ and $u^{\prime} \Psi_{1}=\exp \left\{-i\left(h_{1}^{\prime}-h_{2}^{\prime}\right)\right\} \Psi_{1}$ due to $j_{\Psi_{1}}\left(h_{1}-h_{2}\right) \Psi_{1}=\left(h_{1}^{\prime}-h_{2}^{\prime}\right) \Psi_{1}$, we obtain

$$
\begin{gathered}
\Phi_{2}=\Psi_{2}+t_{2} \Psi_{2}, \\
\omega_{\Phi_{2}}=\omega_{\Phi_{1}} .
\end{gathered}
$$

We have

$$
S_{1}=1+t_{1+}+(i / 2)\left\{\left(h_{1}-h_{2}\right)-\left(h_{1}^{\prime}-h_{2}^{\prime}\right)\right\} .
$$

Hence $\tau_{\Psi_{1}}(-i / 2) S_{1}^{*}=S_{1}$ and $\left(\tau_{\Psi_{1}}(i / 4) S_{1}\right)$ is symmetric. Furthermore, 
$(6.15)$

$$
\left\|t_{1+}+(i / 2)\left\{\left(h_{1}-h_{2}\right)-\left(h_{1}^{\prime}-h_{2}^{\prime}\right)\right\}\right\| \leqq 3 \delta<1 .
$$

Hence $\left\|\tau_{\Psi_{1}}(-i / 4)\left(S_{1}-1\right)^{*}\right\| \leqq 3 \delta$ and $\tau_{\Psi_{1}}(i / 4) S_{1} \geqq 0$. Therefore $\Psi_{2} \in$ $V_{\Psi_{1}}=V_{\Psi}$.

Since $S_{1}$ is invertible, $\Psi_{2}$ is again cyclic and separating.

We have

$$
\begin{gathered}
\left\|t_{2}^{\prime}\right\| \leqq(1+\delta)\left(e^{3 \delta}-1-3 \delta\right)+3 \delta^{2}, \\
\left\|S_{1}^{-1}\right\| \leqq(1-3 \delta)^{-1} .
\end{gathered}
$$

Hence

$$
\left\|t_{2}\right\| \leqq a_{1} \delta^{2}
$$

with

$$
\begin{aligned}
a_{1} & \equiv(1-3 \delta)^{-1}\left\{3+(1+\delta)\left(e^{3 \delta}-1-3 \delta\right) / \delta^{2}\right\} \\
& \leqq(1-3 \delta)^{-1}\left(3+(9 / 2)(1+\delta) e^{3 \delta}\right)<16
\end{aligned}
$$

for $\delta \leqq 2^{-4}$. Hence

$$
\left\|t_{2}\right\| \leqq a \delta
$$

with $a=a_{1} 2^{-4}<1$.

By Lemma 7 ,

$$
\tau_{\Psi_{2}}(-i / 2)\left(t_{2}^{*}\right)=S_{1}\left\{\tau_{\Psi_{1}}(-i / 2)\left(S_{1}^{*-1} t_{2}^{\prime *}\right)\right\} S_{1}^{-1} .
$$

Since $\tau_{\Psi_{1}}(-i / 2) S_{1}^{*}=S_{1}$,

$$
\begin{aligned}
& \tau_{\Psi_{2}}(-i / 2)\left(t_{2}^{*}\right)=\left\{\tau_{\Psi_{1}}(-i / 2)\left(t_{2}^{*}\right)\right\} S_{1}^{-1} \\
&=\left\{\left(-1-i\left(h_{1}-h_{2}\right)+\exp \left\{i\left(h_{1}-h_{2}\right)\right\}\right)\left(1+\tau_{\Psi_{1}}(-i / 2)\left(t_{1}^{*}\right)\right)\right. \\
&\left.+i\left(h_{1}-h_{2}\right) \tau_{\Psi_{1}}(-i / 2)\left(t_{1}^{*}\right)\right\} S_{1}^{-1} .
\end{aligned}
$$

Therefore,

$$
\begin{aligned}
\left\|\tau_{\Psi_{2}}(-i / 2)\left(t_{2}^{*}\right)\right\| & \leqq\left\{(1+\delta)\left(e^{\delta}-1-\delta\right)+\delta^{2}\right\}(1-3 \delta)^{-1} \\
& \leqq a \delta
\end{aligned}
$$

From (6.15), we also have

$$
\begin{aligned}
\left\|\Psi_{1}-\Psi_{2}\right\| & \leqq\left\|1-S_{1}\right\|\left\|\Psi_{1}\right\| \\
& \leqq\left\|1-S_{1}\right\|\left\|\left(1+t_{1}\right)^{-1}\right\| \omega_{\Phi_{1}}(1)^{1 / 2} \\
& \leqq 3 \delta(1-\delta)^{-1} \omega_{\emptyset_{1}}(1)^{1 / 2} \\
& \leqq 4 \delta \omega_{\Phi_{1}}(1)^{1 / 2} .
\end{aligned}
$$

We can now repeat the process and obtain a sequence of vectors $\Phi_{n}, \Psi_{n}$ and operators $t_{n} \in R$ such that $\Psi_{n}$ is cyclic and separating, $\Psi_{n} \in V_{\Psi}$, 


$$
\begin{gathered}
\Phi_{n}=\Psi_{n}+t_{n} \Psi_{n}, \\
\left\|t_{n}\right\| \leqq a^{n-1} \delta, \quad\left\|\tau_{\Psi_{n}}(-i / 2)\left(t_{n}^{*}\right)\right\| \leqq a^{n-1} \delta, \\
\omega_{\Phi_{n}}=\omega_{\Phi_{1}}, \\
\left\|\Psi_{n}-\Psi_{n-1}\right\| \leqq 4 a^{n-2} \delta \omega_{\Phi_{1}}(1)^{1 / 2} .
\end{gathered}
$$

$\Psi_{n}$ is a Cauchy sequence and has a limit

$$
\Phi=\lim \Psi_{n} \in V_{\Psi} .
$$

Since $\lim \left\|t_{n} \Psi_{n}\right\|=0$, we have

$$
\begin{gathered}
\Phi=\lim \Phi_{n}, \\
\omega_{\Phi}=\lim \omega_{\Phi_{n}}=\omega_{\Phi_{1}} .
\end{gathered}
$$

Step (ii). We prove that if $t^{*}=t \in R$ and $\tau_{\Psi}(z) t \in R$ for $\operatorname{Im} z \epsilon$ $[-1,1]$, then there exists $\Phi \in V_{\Psi}$ such that $\omega_{\Phi}=\omega_{(\exp t) \Psi}$.

Let $x(\lambda) \equiv(\exp \lambda t) \Psi, 0 \leqq \lambda \leqq 1$. It is cyclic and separating because $\Psi$ is cyclic and separating and $e^{\lambda t}$ is invertible. We have

$$
\begin{aligned}
J_{x(\lambda)} \Delta_{x(\lambda)}^{1 / 2} t x(\lambda) & =t x(\lambda)=e^{\lambda t} t \Psi \\
& =e^{\lambda t} J_{\Psi}\left\{\tau_{\Psi}(-i / 2) t\right\} \Psi=t^{\prime} e^{\lambda t} \Psi
\end{aligned}
$$

where $t^{\prime} \equiv j_{\Psi}\left\{\tau_{\Psi}(-i / 2) t\right\} \in R^{\prime}$. Then

$$
\begin{aligned}
& \Delta_{x(\lambda)}^{1 / 2} J_{x(\lambda)} t^{\prime} x(\lambda)=t^{\prime *} x(\lambda)=e^{\lambda t} t^{* *} \Psi \\
& \quad=e^{\lambda t} J_{\Psi} \Delta_{\Psi}^{-1 / 2} t^{\prime} \Psi=e^{\lambda t} \Delta_{\Psi} t \Psi=t^{\prime \prime} x(\lambda)
\end{aligned}
$$

where $t^{\prime \prime}=e^{\lambda t}\left\{\tau_{\Psi}(-i) t\right\} e^{-\lambda t}$. Combining two computations, we have

$$
\Delta_{x(\lambda)} t x(\lambda)=t^{\prime \prime} x(\lambda) \text {. }
$$

By Lemma $6, \tau_{x(\lambda)}(z) t \in R$ for $\operatorname{Im} z \in[-1,0]$. Since $\left(\tau_{x(\lambda)}(\bar{z}) t\right)^{*}$ is holomorphic for $\operatorname{Im} z \in(0,1)$ and coincides with $\tau_{x(\lambda)}(z) t$ at $\operatorname{Im} z=0$, it is an analytic continuation of $\tau_{x(\lambda)}(z) t$. We have $\tau_{x(\lambda)}(z) \in R$ for $\operatorname{Im} z \in[-1,1]$ and $\left\|\tau_{x(\lambda)}(z) t\right\| \leqq\left\|t^{\prime \prime}\right\|$. We note that $\|t\|=\left\|\tau_{x(\lambda)}(0) t\right\| \leqq\left\|t^{\prime \prime}\right\|$.

For $y \in D_{x(2)}$ we have convergence of

$$
\sum_{n=0}^{\infty}(n !)^{-1}\left(\lambda^{\prime} t\right)^{n} \Delta_{x(\lambda)}^{-i z} y=e^{\lambda^{\prime} t} \Delta_{x(\lambda)}^{-i z} y,
$$

and

$$
\sum_{n=c}^{\infty}(n !)^{-1} \Delta_{x(\lambda)}^{i z}\left(\lambda^{\prime} t\right)^{n} \Delta_{x(\lambda)}^{-i z} y=\exp \left\{\lambda^{\prime} \tau_{x(\lambda)}(z) t\right\} y
$$

for $\operatorname{Im} z \in[-1,1]$. Hence

$$
\Delta_{x(\lambda)}^{i z} e^{\lambda^{\prime} t} \Delta_{x(\lambda)}^{-i z} y=\exp \left\{\lambda^{\prime} \tau_{x(\lambda)}(z) t\right\} y .
$$


In particular, for $\lambda^{\prime}>0$,

$$
\left\|\tau_{x(\lambda)}(-i / 2) e^{\lambda^{\prime} t}-1\right\| \leqq e^{\lambda^{\prime}|| t^{\prime \prime} \|}-1 .
$$

Let $N$ be a natural number satisfying

$$
N \geqq 2^{4} C e^{C}, \quad C=e^{2\|t\|}\left\|\tau_{\Psi}(-i) t\right\| \geqq\left\|t^{\prime \prime}\right\| .
$$

Let $\lambda_{n}=n / N$. We have

$$
\begin{aligned}
\left\|e^{\lambda_{1} t}-1\right\| & \leqq e^{\lambda_{1}\|t\|}-1 \\
& \leqq e^{\lambda_{1}\left\|t^{\prime \prime}\right\|}-1 \leqq \lambda_{1}\left\|t^{\prime \prime}\right\| e^{\lambda_{1}|| t^{\prime \prime} \|} \\
& \leqq 2^{-4}
\end{aligned}
$$

Similarly, for $0 \leqq \lambda \leqq 1$,

$$
\left\|\tau_{x(\lambda)}(-i / 2) e^{\lambda_{1} t}-1\right\| \leqq 2^{-4}
$$

In other words, $t^{\prime \prime \prime} \equiv e^{\lambda_{1} t}-1$ satisfies $\left\|t^{\prime \prime \prime}\right\| \leqq 2^{-4}$ and

$$
\left\|\tau_{x(\lambda)}(-i / 2) t^{\prime \prime \prime}\right\| \leqq 2^{-4}
$$

for $0 \leqq \lambda \leqq 1$, and $e^{\lambda_{1} t}=1+t^{\prime \prime \prime}$.

Let $y(n)=\exp (t / N) \Phi(n-1)$, where $\Phi(0) \equiv \Psi$ and $\Phi(n)$ is to be determined inductively such that $\Phi(n) \in V_{\Psi}, \Phi(n)$ is cyclic and separating, $\omega_{\Phi(n)}=\omega_{x\left(\lambda_{n}\right)}$ and $n \leqq N . \quad \Phi(0) \equiv \Psi$ obviously satisfies requirements for $\Phi(n), n=0$.

If $\omega_{\Phi(n-1)}=\omega_{x\left(\lambda_{n-1}\right)}$, then $\omega_{y(n)}=\omega_{\exp (t, N) x\left(\lambda_{n-1}\right)}=\omega_{x\left(\lambda_{n}\right)}$. Since $y(n)=$ $\left(1+t^{\prime \prime \prime}\right) \Phi(n-1)$, we can apply Step (i) if $\Phi(n-1) \in V_{\Psi}$ and $\Phi(n-1)$ is cyclic and separating. There exists $\Phi(n) \in V_{x}$ such that $\omega_{\mathscr{\varphi}(n)}=\omega_{y(n)}=$ $\omega_{x\left(\lambda_{n}\right)}$. Since $x(\lambda)$ is separating, $s^{R}\left(\omega_{\Phi(n)}\right)=1$. Hence $s^{R^{\prime}}(\Phi(n))=$ $j_{\Psi}\left\{s^{R}(\Phi(n))\right\}=1$ due to $\Phi(n) \in V_{\Psi}$. Thus, by induction, we have desired $\Phi(n), n \leqq N$. In particular, $\Phi(N) \in V_{\Psi}$ satisfies $\omega_{\Phi(N)}=\omega_{(\exp t) \Psi}$.

Step (iii). Let $S_{x}$ be the set of all $\omega_{x}, x \in V_{Y}$. $S_{Y}$ is a norm closed subset of $R_{*}^{+}$by (5.10). We prove that any $\rho \in R_{*}^{+}$is in $S_{\Psi}$.

Since $\Psi$ is cyclic and separating, there exists a positive selfadjoint operator $A_{2}$ affiliated with $R$ such that $\Psi$ is in the domain of $A_{2}$ and $\rho=\omega_{A_{2} \Psi}[3]$. Let $A_{2}=\int \lambda \mathrm{d} E_{\lambda}, A_{2}^{L}=A_{2}\left(E_{L}-E_{1 / L}\right)+\left\{1-E_{L}+(1 / \mathrm{L}) E_{1 / L}\right)$, $t=\left(\log A_{2}^{L}\right)\left(f_{\beta}^{G}\right), \rho_{L \beta}=\omega_{(\exp t) \Psi}$. Then $t$ is a selfadjoint element of $\mathfrak{A}_{T_{1}}$. By Step (ii), $\rho_{L \beta} \in S_{\Psi}$. Since $\lim _{L \rightarrow+\infty} \lim _{\beta \rightarrow+0}\left\|\rho_{L \beta}-\rho\right\|=0$, we have $\rho \in S_{\Psi}$.

7. Representation of $R_{*}^{+}$by $V_{\Psi}$. We denote the set of all normal positive linear functionals on $R$ by $R_{*}^{+}$and the set of all normal states on $R$ by $R_{* 1}^{+}$. As before $\omega_{x}$ denotes the expectation functional by a vector $x$. 
THEOREM 7. Assume that $R$ and $R_{\alpha}$ have cyclic and separating vectors $\Psi$ and $\Psi_{\alpha}$, respectively.

(1) The mapping $\sigma_{\Psi}$ from $\omega_{x} \in R_{*}^{+}$to $\sigma_{\Psi}\left(\omega_{x}\right) \equiv x \in V_{\Psi}$ is a bijective homeomorphism from $R_{*}^{+}$onto $V_{\Psi}$ relative to the norm topologies.

(2) If $\rho=\sum_{n} \rho_{n}, \rho \in R_{*}^{+}, \rho_{n} \in R_{*}^{+}$and $s\left(\rho_{n}\right)$ are mutually orthogonal, then $\sigma_{\Psi} \rho=\sum \sigma_{\Psi} \rho_{n}$.

(3) If $R=\bigoplus_{n} R_{n}, \Psi=\bigoplus \Psi_{n}$, then $\sigma_{\Psi}\left(\bigoplus \rho_{n}\right)=\bigoplus \rho_{\Psi_{n}}\left(\rho_{n}\right)$ for any $\rho_{n} \in\left(R_{n}\right)_{*}^{+}, \oplus \rho_{n} \in R_{*}^{+}$.

(4) If $R=\otimes\left(R_{\alpha}, \Psi_{\alpha}\right)$ on $H=\bigotimes\left(H_{\alpha}, \Psi_{\alpha}\right)$ (the incomplete infinite tensor product containing $\left.\Psi \equiv \otimes \Psi_{\alpha}\right)$, then $\sigma_{\Psi}\left(\otimes \rho_{\alpha}\right)=\otimes \sigma_{\Psi_{\alpha}}\left(\rho_{\alpha}\right)$ if $\rho_{\alpha} \in\left(R_{\alpha}\right)_{* 1}^{+}$and $\otimes \sigma_{y}\left(\rho_{\alpha}\right) \in \otimes\left(H_{\alpha}, \Psi_{\alpha}\right)$. The last condition is equivalent to existence of $\rho \in R_{*}^{+}$such that

$$
\rho\left(Q \otimes\left(\bigotimes_{\alpha \notin J} 1_{\alpha}\right)\right)=\left(\bigotimes_{\alpha \in J} \rho_{\alpha}\right)(Q), \quad Q \in \bigotimes_{\alpha \in J} R_{\alpha}
$$

for every finite index set $J . \quad$ (Symbolically $\otimes \rho_{\alpha} \in R_{*}^{+}$.)

(5) For any $\Phi \in H$, there exists a unique $|\Phi|_{Y} \in V_{\Psi}$ and a partial isometry $u^{\prime} \in R^{\prime}$ such that

$$
\begin{gathered}
\Phi=u^{\prime}|\Phi|_{\Psi}, \\
u^{\prime} u^{\prime *}=s^{R^{\prime}}(\Phi), \quad u^{\prime *} u^{\prime}=s^{R^{\prime}}\left(|\Phi|_{\Psi}\right) .
\end{gathered}
$$

There also exist a unique $|\Phi|_{\Psi}^{\prime} \in V_{\Psi}$ and a partial isometry $u \in R$ such that

$$
\begin{gathered}
\Phi=u|\Phi|_{\Psi}^{\prime}, \\
u u^{*}=s^{R}(\Phi), \quad u^{*} u=s^{R}\left(|\Phi|_{\psi}^{\prime}\right) .
\end{gathered}
$$

They are related by

$$
u=j_{\Psi}\left(u^{\prime}\right)^{*}, \quad|\Phi|_{\Psi}^{\prime}=u^{\prime} j_{\Psi}\left(u^{\prime}\right)|\Phi|_{\Psi} .
$$

(6) If $\Phi$ is any cyclic and separating vector for $R$, there exists a unitary $w \in R^{\prime}$ such that

$$
\sigma_{\Psi}(\rho)=w \sigma_{\oplus}(\rho)
$$

for all $\rho \in R_{*}^{+}$.

Proof. (1) follows from Theorem 6, (5.10) and

$$
\begin{aligned}
\left|\omega_{x}(Q)-\omega_{y}(Q)\right| & =|(x+y, Q(x-y))+(x-y, Q(x+y))| / 2 \\
& \leqq\|x+y\|\|x-y\|\|Q\|,
\end{aligned}
$$

which implies

$$
\left\|\omega_{x}-\omega_{y}\right\| \leqq\|x+y\|\|x-y\|
$$


(2) By (1), there exists $\Phi_{n} \in V_{\Psi}$ such that $\omega_{\Phi_{n}}=\rho_{n}$. Since $s\left(\rho_{n}\right)$ are mutually orthogonal, $s^{R}\left(\Phi_{n}\right)=s\left(\rho_{n}\right)$ are mutually orthogonal and

$$
\sum\left\|\Phi_{n}\right\|^{2}=\sum \rho_{n}(1)=\rho(1)<\infty .
$$

Hence we have convergence of

$$
\Phi=\sum \Phi_{n} .
$$

Since $\Phi_{n} \in V_{\Psi}, s^{R^{\prime}}\left(\Phi_{n}\right)=j_{\Psi}\left(s^{R}\left(\Phi_{n}\right)\right)$ are also mutually orthogonal. Hence

$$
\begin{aligned}
\left(\Phi_{n}, Q \Phi_{m}\right) & =\left(\Phi_{n}, Q s^{R^{\prime}}\left(\Phi_{m}\right) \Phi_{m}\right) \\
& =\left(s^{R^{\prime}}\left(\Phi_{m}\right) \Phi_{n}, Q \Phi_{m}\right)=0
\end{aligned}
$$

for $Q \in R$ and $m \neq n$. Therefore,

$$
(\Phi, Q \Phi)=\sum\left(\Phi_{n}, Q \Phi_{n}\right)=\sum \rho_{n}(Q)=\rho(Q) .
$$

Hence $\Phi=\sigma_{\Psi} \rho=\sum \sigma_{\Psi} \rho_{n}$.

(3) This follows from (2).

(4) If $\Psi=\bigotimes \Psi_{\alpha}$, then $J_{\Psi}=\bigotimes J_{\Psi_{\alpha}}$ and $\Delta_{\Psi}=\bigotimes \Delta_{\Psi_{\alpha}}$ which is seen as follows: Let $J=\bigotimes J_{\psi_{\alpha}}, \Delta^{i t}=\bigotimes \Delta_{\psi_{\alpha}}^{i t}$. Then $J \Delta^{1 / 2} Q \Psi=Q^{*} \Psi$ if $Q=$ $\otimes Q_{\alpha}$ and $Q_{\alpha}=1$ except for a finite number of $\alpha$. Since such $Q$ is * strongly total in $R, J \Delta^{1 / 2} Q \Psi=Q^{*} \Psi$ for any $Q \in R$ and hence $J \Delta^{1 / 2} \supset$ $J_{\Psi} \Delta_{\Psi}^{1 / 2} . \quad J$ satisfies (i)-(iv) of Theorem 1 . It also satisfies (v) due to $J Q^{*} \Psi=\Delta^{1 / 2} Q \Psi$ and $\Delta \geqq 0$. Hence $J=J_{\Psi}$. Hence $\Delta=\Delta_{\Psi}$.

If $\otimes \sigma_{\Psi_{\alpha}}\left(\rho_{\alpha}\right) \in \otimes\left(H_{\alpha}, \Psi_{\alpha}\right)$ and $\rho_{\alpha}$ are faithful, then

$$
J_{\otimes \sigma_{\Psi_{\alpha}}}\left(\rho_{\alpha}\right)=\bigotimes J_{{\sigma_{\Psi}}_{\alpha}\left(\rho_{\alpha}\right)}=\bigotimes J_{\Psi_{\alpha}}=J_{\otimes \Psi_{\alpha}} .
$$

Let $Z_{\alpha}$ be the center of $R_{\alpha}$. Then $\left\{\otimes\left(R_{\alpha}, \Psi_{\alpha}\right)\right\}^{\prime}=\bigotimes\left(R_{\alpha}^{\prime}, \Psi_{\alpha}\right)$ and hence the center $Z$ of $\otimes\left(R_{\alpha}, \Psi_{\alpha}\right)$ is given by $\otimes\left(Z_{\alpha}, \Psi_{\alpha}\right)$. If $z_{\alpha}$ is a projection in $Z_{\alpha}$ and $z_{\alpha}=1$ except for a finite number of $\alpha$, then $z=\otimes z_{\alpha} \in Z$ satisfies

$$
\left(\Psi, z\left\{\bigotimes \sigma_{\Psi_{\alpha}}\left(\rho_{\alpha}\right)\right\}\right)=\Pi\left(\Psi_{\alpha}, z \sigma_{\Psi_{\alpha}}\left(\rho_{\alpha}\right)\right) \geqq 0 .
$$

$Z_{\alpha}$ and $Z$ can be viewed as $L^{\infty}\left(\Xi_{\alpha}, \mu_{\alpha}\right)$ and $L^{\infty}\left(\Pi \Xi_{\alpha}, \otimes \mu_{\alpha}\right)$ where projections are characteristic functions. Hence any projection in $Z$ can be weakly approximated by a finite sum of projections $z=\bigotimes z_{\alpha}$. This implies

$$
\left(\Psi, z\left\{\otimes \sigma_{\Psi_{\alpha}}\left(\rho_{\alpha}\right)\right\}\right) \geqq 0
$$

for all projections in $Z$ and hence for all $z \in Z, z \geqq 0$.

By Theorem 4 (5), we have $\otimes \sigma_{\Psi_{\alpha}}\left(\rho_{\alpha}\right) \in V_{\otimes_{\Psi}}$. The same conclusion holds for nonfaithful $\rho_{\alpha}$, by taking a limit of faithful $\rho_{\alpha}+\lambda_{\alpha} \omega_{\Psi_{\alpha}}$, $\lambda_{\alpha} \geqq 0$ as $\sum \lambda_{\alpha} \rightarrow 0 . \quad\left(\otimes \sigma_{y}\left(\rho_{\alpha}\right) \in \otimes\left(H_{\alpha}, \Psi_{\alpha}\right)\right.$ implies $\sigma_{y}\left(\rho_{\alpha}\right)=\Psi_{\alpha}$ except for a countable number of $\alpha$.) We also have 


$$
\bigotimes \rho_{\alpha} \equiv \omega_{\otimes \sigma_{Y_{\alpha}}}\left(\rho_{\alpha}\right) \in R_{*}^{+}
$$

Hence

$$
\sigma_{\underline{y}}\left(\otimes \rho_{\alpha}\right)=\otimes \sigma_{\Psi_{\alpha}}\left(\rho_{\alpha}\right)
$$

Next assume $\boldsymbol{\otimes} \rho_{\alpha} \in R_{*}^{+}$. Without loss of generality we may assume $\left\|\Psi_{\alpha}\right\|=1$. Let $R(I)=\bigotimes_{\alpha \in I} R_{\alpha}, \Psi(I)=\bigotimes_{\alpha \in I} \Psi_{\alpha}, \Phi_{\alpha}=\sigma_{\Psi_{\alpha}}\left(\rho_{\alpha}\right)$, $\Phi=\sigma_{\Psi}\left(\otimes \rho_{\alpha}\right), \rho_{0}(I)=\omega_{\Psi(I)}$ for an arbitrary index set $I$ and $\rho(J)=$ $\bigotimes_{\alpha \in J} \rho_{\alpha}, \Phi(J)=\bigotimes_{\alpha \in J} \Phi_{\alpha}$ for a finite index set $J . \quad J^{c}$ denotes the complement of $J$ in the index set. $\rho_{\alpha} \in R_{* 1}^{+}$implies

$$
\left\|\Phi_{\alpha}\right\|=\|\Phi(J)\|=\|\Phi\|=1 .
$$

Since $\Psi\left(J^{c}\right) \otimes z$ is total when $J$ runs over finite index sets and $z$ runs over $\boldsymbol{\bigotimes}_{\alpha \in J} H_{\alpha}$, there exists a finite index set $J$ and a $z \in \boldsymbol{\bigotimes}_{\alpha \in J} H_{\alpha}$ such that $\left(\Phi, \Psi\left(J^{C}\right) \otimes z\right) \neq 0,\|z\|=1$. Then for any $K \subset J^{C}$, we have

$$
\left\|\rho(K)-\rho_{0}(K)\right\|=\left\|\omega_{\Phi}^{R(K)}-\omega_{\psi_{(J}\left(J^{C}\right) \otimes z}^{R(K)}\right\|<2 .
$$

(If $(x, y) \neq 0$, then (7.7) implies $\left\|\omega_{x}-\omega_{y^{\prime}}\right\|^{2} \leqq\left(\|x\|^{2}+\left\|y^{\prime}\right\|^{2}\right)^{2}-4\left(x, y^{\prime}\right)^{2}$ for $y^{\prime}=e^{i \theta} y$ where $\theta$ is a real number such that $\left(x, y^{\prime}\right)>0$. Hence $\left\|\omega_{x}-\omega_{y}\right\|<\|x\|^{2}+\|y\|^{2}$.)

By the first part of the proof of (4), we have $\sigma_{\Psi(K)}(\rho(K))=$ $\boldsymbol{\bigotimes}_{\alpha \in K} \sigma_{\Psi_{\alpha}}\left(\rho_{\alpha}\right)=\Phi(K)$ for a finite index set $K$ where the condition $\boldsymbol{\bigotimes}_{\alpha \in K} \sigma_{\Psi_{\alpha}}\left(\rho_{\alpha}\right) \in \boldsymbol{\bigotimes}_{\alpha \in K} H_{\alpha}$ is trivially satisfied. By (5.10)

$$
\|\Psi(K)-\Phi(K)\|^{2} \leqq\left\|\rho(K)-\rho_{0}(K)\right\|
$$

and hence

$$
(\Psi(K), \Phi(K)) \geqq 2^{-1}\left(2-\left\|\rho(K)-\rho_{0}(K)\right\|\right) \equiv \delta>0,
$$

where we have used $(\Psi(K), \Phi(K)) \geqq 0$ due to $\Phi(K) \in V_{\Psi_{(K)}}$. Since $\left\|\Psi_{\alpha}\right\|=\left\|\Phi_{\alpha}\right\|=1$, we have $1 \geqq\left(\Psi_{\alpha}, \Phi_{\alpha}\right)>0$ and hence

$$
1 \geqq \prod_{\alpha \in K}\left(\Psi_{\alpha}, \Phi_{\alpha}\right) \geqq \hat{\delta}>0
$$

for any finite index set $K \subset J^{C}$. Hence

$$
\sum_{\alpha}\left|1-\left(\Psi_{\alpha}, \Phi_{\alpha}\right)\right|<\infty
$$

which implies $\otimes \Phi_{\alpha} \in \bigotimes\left(H_{\alpha}, \Psi_{\alpha}\right)$.

Therefore, $\otimes \rho_{\alpha} \in R_{*}^{+}$implies $\otimes \sigma_{\Psi_{\alpha}}\left(\rho_{\alpha}\right) \in \otimes\left(H_{\alpha}, \Psi_{\alpha}\right)$.

(5) For any $\Phi \in H$, there exists a unique $|\Phi|_{\Psi} \in V_{\Psi}$ satisfying $\omega_{\Phi}=\omega_{\left.i\right|_{\Psi}}$ by (1). Then there exists a unique partial isometry $u^{\prime} \in R^{\prime}$ satisfying (7.1) and (7.2).

Next set

$$
|\Phi|_{\Psi}^{\prime} \equiv u^{\prime} j_{\Psi}\left(u^{\prime}\right)|\Phi|_{\Psi}
$$


Then $|\Phi|_{\Psi}^{\prime}=j_{\Psi}\left(u^{\prime}\right) \Phi$. Since $s^{R}\left(|\Phi|_{\Psi}\right)=j_{\Psi}\left(s^{R^{\prime}}\left(|\Phi|_{\Psi}\right)\right)=j_{\Psi}\left(u^{\prime *} u^{\prime}\right)$, we have

$$
\Phi=u^{\prime}|\Phi|_{\Psi}=j_{\Psi}\left(u^{\prime}\right)^{*}|\Phi|_{\Psi}^{\prime} \text {. }
$$

We also have

$$
\begin{aligned}
j_{\Psi}\left(u^{\prime}\right) j_{\Psi}\left(u^{\prime}\right)^{*} & =j_{\Psi}\left(s^{R^{\prime}}(\Phi)\right)=j_{\Psi}\left\{s\left(\omega_{\Phi}^{R^{\prime}}\right)\right\}=j_{\Psi}\left\{s^{R^{\prime}}\left(\omega_{\mid \Phi_{\mid \Psi}}^{R^{\prime}}\right)\right\} \\
& =j_{\Psi}\left\{s^{R^{\prime}}\left(|\Phi|_{\Psi}^{\prime}\right)\right\}=s^{R}\left(|\Phi|_{\Psi}^{\prime}\right)
\end{aligned}
$$

where the last equality is due to $|\Phi|_{\Psi}^{\prime} \in V_{\Psi}$ and $\omega_{x}^{R^{\prime}}$ denotes the expectation functional on $R^{\prime}$ by a vector $x$.

Thus (7.5) satisfies (7.3) and (7.4).

To see the uniqueness of $|\Phi|_{\varphi}^{\prime}$ and $u$, we note $\omega_{\phi}^{R^{\prime}}=\omega_{||_{\mid}^{\prime}}^{R^{\prime}}$. If we interchange the role of $R$ and $R^{\prime}$ in the definition of $V_{\psi}$, we obtain the same set $V_{\Psi}$. Hence by (1), a vector $x \in V_{\Psi}$ satisfying $\omega_{x}^{R^{\prime}}=\rho$ for any given $\rho \in\left(R^{\prime}\right)_{*}^{+}$is unique. Hence the uniqueness of $|\Phi|_{\Psi}^{\prime}$. The unitary operator $u \in R$ satisfying (7.3) and (7.4) is unique because $u Q|\Phi|_{\Psi}^{\prime}=Q \Phi$ for $Q \in R^{\prime}$ determines $u$ on $s^{R}\left(|\Phi|_{\Psi}^{\prime}\right)$.

(6) Since $\Phi$ is separating $s^{R}\left(\sigma_{\Psi} \omega_{\oplus}\right)=s\left(\omega_{\emptyset}\right)=1$. Hence $s^{R^{\prime}}\left(\sigma_{\psi} \omega_{\oplus}\right)=$ $j_{\Psi}\left\{s^{R}\left(\sigma_{\Psi} \omega_{\Phi}\right)\right\}=1$ and $\sigma_{\Psi} \omega_{\Phi}$ is cyclic and separating. By Corollary 2 of $\S 4, J_{\sigma_{\Psi} \omega_{\Phi}}=J_{\Psi}$ and $V_{\text {ơw } \omega_{\Phi}}=V_{\Psi}$.

Since $\omega_{\Phi}=\omega_{\text {oy } \omega_{\Phi}}$, there exists a partial isometry $w \in R^{\prime}$ such that $\sigma_{\Psi} \omega_{\Phi}=w \Phi$. Since both $\Phi$ and $\sigma_{\Psi} \omega_{\Phi}$ are cyclic, $w$ is unitary.

Since $w \in R^{\prime}$, we have for $S=J_{w \Phi} \Delta_{w \Phi}^{1 / 2}$ and $S_{\Phi}=J_{\Phi} \Delta_{\Phi}^{1 / 2}$,

$$
S w Q \Phi=S Q w \Phi=Q^{*} w \Phi=w Q^{*} \Phi=w S_{\Phi} Q \Phi, \quad Q \in R .
$$

Hence $S=w S_{\Phi} w^{*}$ and $J_{\Psi}=J_{w \Phi}=w J_{\Phi} w^{*}$. Hence

$$
\left(w \sigma_{\oplus} \rho, Q j_{\Psi}(Q) w \Phi\right)=\left(\sigma_{\varphi} \rho, Q j_{\Phi}(Q) \Phi\right) \geqq 0 .
$$

By Theorem 4 (1) and (4),

$$
w \sigma_{\oplus} \rho \in V_{w \Phi}=V_{\Psi} .
$$

By the uniqueness in (1), $w \sigma_{\Phi} \rho=\sigma_{\varphi} \rho$.

8. Applications of $\sigma_{\Psi}$. The following theorems are examples of applications of Theorem 7 .

THEOREM 8. Let $\Psi$ and $\Phi$ be cyclic and separating vectors for $R$. Then the * automorphism

$$
Q \in R \rightarrow j_{\Psi}\left\{j_{\phi}(Q)\right\} \in R
$$

of $R$ is inner. ${ }^{1}$

1 The author is informed by Professor Takesaki that Dr. Connes has a simple proof of this. 
Proof. By the proof of Theorem 7 (6),

$$
J_{\Psi}=w J_{\Phi} w^{*}
$$

for a unitary $w \in R^{\prime}$. Setting $u=j_{\Phi}\left(w^{*}\right)$, we have

$$
j_{\Psi}\left\{j_{\Phi}(Q)\right\}=u Q u^{*}
$$

where $u$ is unitary and $u \in R$.

THEOREM 9. Let $\mathfrak{A}$ be the $C^{*}$ algebra inductive limit of finite $W^{*}$ tensor products $\left\{\bigotimes_{\alpha \in J} R_{\alpha}\right\} \equiv R(J)$, where $J$ is any finite subset of given index set $\{\alpha\}$. Let $\rho_{\alpha}, \rho_{\alpha}^{\prime} \in\left(R_{\alpha}\right)_{* 1}^{+}$. Assume that central supports of $\rho_{\alpha}$ and $\rho_{\alpha}^{\prime}$ are the same. The representations of $\{$ canonically associated with $\otimes \rho_{\alpha}$ and $\otimes \rho_{\alpha}^{\prime}$ are quasi-equivalent, if and only if $\sum d^{\prime}\left(\rho_{\alpha}, \rho_{\alpha}^{\prime}\right)^{2}<\infty$, where

$$
d^{\prime}\left(\rho_{\alpha}, \rho_{\alpha}^{\prime}\right) \equiv\left\|\sigma_{\Psi_{\alpha}}\left(\rho_{\alpha}\right)-\sigma_{\Psi_{\alpha}}\left(\rho_{\alpha}^{\prime}\right)\right\|
$$

does not depend on $\Psi_{\alpha}$.

Proof. By Theorem $7(6), d^{\prime}\left(\rho_{1}, \rho_{2}\right)$ does not depend on $\Psi$.

First assume $\sum d^{\prime}\left(\rho_{\alpha}, \rho_{\alpha}^{\prime}\right)^{2}<\infty$. Then there exists a countable index set $I$ such that $d^{\prime}\left(\rho_{\alpha}, \rho_{\alpha}^{\prime}\right)=0$ for $\alpha \notin I$. Then $\rho_{\alpha}=\rho_{\alpha}^{\prime}$ for $\alpha \notin I$.

By assumption

$$
\sum_{\alpha}\left|1-\left(\sigma_{\Psi_{\alpha}} \rho_{\alpha}, \sigma_{\Psi_{\alpha}} \rho_{\alpha}^{\prime}\right)\right|<\infty .
$$

Hence $\Phi \equiv \bigotimes_{\alpha} \sigma_{\Psi_{\alpha}} \rho_{\alpha}$ and $\Phi^{\prime} \equiv \bigotimes_{\alpha} \sigma_{\Psi_{\alpha}} \rho_{\alpha}^{\prime}$ belong to the same incomplete infinite tensor product $H=\otimes\left(H_{\alpha}, \sigma_{\Psi_{\alpha}} \rho_{\alpha}\right)$. The $C^{*}$ algebra $\mathfrak{A}$ has a natural representation $\pi$ on $H$ and $\otimes \rho_{\alpha}=\omega_{\Phi}, \otimes \rho_{\alpha}^{\prime}=\omega_{\Phi^{\prime}}$. Let $E_{\alpha}$ be the central support of $\rho_{\alpha}$, which is the same as the central support of $\rho_{\alpha}^{\prime}$. Then $\left(R_{\alpha} \cup R_{\alpha}^{\prime}\right) \sigma_{\Psi_{\alpha}} \rho_{\alpha}=E_{\alpha} H_{\alpha}$. Since $\left(\otimes R_{\alpha}\right)^{\prime}=\otimes R_{\alpha}^{\prime}$ in an incomplete infinite tensor product, the central support $E$ of $\otimes \sigma_{\Psi_{\alpha}} \rho_{\alpha}$ satisfies $E H=\lim _{J \uparrow}\left(\bigotimes_{\alpha \notin J} \sigma_{\Psi_{\alpha}} \rho_{\alpha}\right) \otimes\left(\bigotimes_{\alpha \in J} E_{\alpha} H_{\alpha}\right)$. By the same calculation the central support of $\otimes \sigma_{\Psi_{\alpha}} \rho_{\alpha}^{\prime}$ coincides with $E$. Hence $\otimes \rho_{\alpha}$ and $\otimes \rho_{\alpha}^{\prime}$ produce quasi-equivalent representations of $\mathfrak{A}$.

Next assume that representations of $\mathfrak{A}$ associated with $\otimes \rho_{\alpha}$ and $\otimes \rho_{\alpha}^{\prime}$ are quasi-equivalent. Let $H_{\alpha}, \pi_{\alpha}, \Phi_{\alpha}$ be canonically associated with $\rho_{\alpha}$. We have $\omega_{\Phi}=\otimes \rho_{\alpha}$ for $\Phi=\otimes \Phi_{\alpha}$.

By assumption of quasi-equivalence, there exists $x_{n} \in \mathbb{\otimes}\left(H_{\alpha}, \Phi_{\alpha}\right)$, $x_{1} \neq 0$ such that $\otimes \rho_{\alpha}^{\prime}=\sum_{n} \omega_{x_{n}}$. Since $\left(\otimes_{\alpha \notin J} \Phi_{\alpha}\right) \otimes z$ is total when $J$ runs over all finite index sets and $z$ runs over $\otimes_{\alpha \in J} H_{\alpha}$, there exists a finite index set $J$ and $z \in \bigotimes_{\alpha \in J} H_{\alpha}$ such that $\left(\mathrm{x}_{1},\left(\bigotimes_{\alpha \notin J} \Phi_{\alpha}\right) \otimes z\right) \neq 0$. Denote $\rho^{\prime}=\otimes \rho_{\alpha}^{\prime}$ and $\rho^{\prime \prime}=\omega_{\left(\otimes_{\alpha \notin J} \Phi_{\alpha}\right) \otimes z}$. Then $\left\|\rho^{\prime}-\rho^{\prime \prime}\right\|<2$.

Let $\rho_{K}=\bigotimes_{\alpha \in K} \rho_{\alpha}, \rho_{K}^{\prime}=\bigotimes_{\alpha \in K} \rho_{\alpha}^{\prime}$. Restrictions of $\rho^{\prime \prime}$ and $\rho^{\prime}$ to $\boldsymbol{\otimes}_{\alpha \in K} R_{\alpha}$ is $\rho_{K}$ and $\rho_{K}^{\prime}$ for any finite index set $K$ in $J^{c}$. By Theorem 7 
(4) and (3.10), we have

$$
\begin{aligned}
\prod_{\alpha \in K}\left(\sigma_{\Psi_{\alpha}} \rho_{\alpha}, \sigma_{\Psi_{\alpha}} \rho_{\alpha}^{\prime}\right) & =\left\{2-\left\|\sigma_{\Psi(K)} \rho_{K}-\sigma_{\Psi(K)} \rho_{K}^{\prime}\right\|^{2}\right\} / 2 \\
& \geqq\left\{2-\left\|\rho^{\prime \prime}-\rho^{\prime}\right\|\right\} / 2>0
\end{aligned}
$$

where $\Psi(K)=\bigotimes_{\alpha \in K} \Psi_{\alpha}$. Since $0 \leqq\left(\sigma_{\Psi_{\alpha}} \rho_{\alpha}, \sigma_{\Psi_{\alpha}} \rho_{\alpha}^{\prime}\right) \leqq 1$, we have

$$
2 \sum\left|1-\left(\sigma_{\Psi_{\alpha}} \rho_{\alpha}, \sigma_{\Psi_{\alpha}} \rho_{\alpha}^{\prime}\right)\right|=\sum\left\|\sigma_{\Psi_{\alpha}} \rho_{\alpha}-\sigma_{\Psi_{\alpha}} \rho_{\alpha}^{\prime}\right\|^{2}<\infty .
$$

REMARK 1. The distance $d^{\prime}\left(\rho, \rho^{\prime}\right)$ satisfies

$$
d^{\prime}\left(\rho, \rho^{\prime}\right) \geqq d\left(\rho, \rho^{\prime}\right)
$$

where $d\left(\rho, \rho^{\prime}\right)$ is the Bures distance [5]. Since $\sum d\left(\rho_{\alpha}, \rho_{\alpha}^{\prime}\right)^{2}<\infty$ is another necessary and sufficient condition for quasi-equivalence, it must be equivalent to $\sum d^{\prime}\left(\rho_{\alpha}, \rho_{\alpha}^{\prime}\right)^{2}<\infty$. Hence there must be a constant $\lambda>1$, such that

$$
\lambda d\left(\rho, \rho^{\prime}\right) \geqq d^{\prime}\left(\rho, \rho^{\prime}\right) .
$$

REMARK 2. If $R$ is semifinite, $\varphi$ if a $\sigma$-finite faithful normal trace on $R, H$ is the Hilbert space of Hilbert-Schmidt operator affiliated with $R$, Hilbert-Schmidt relative to $\varphi$, and $R$ is left multiplication, then an example of $V_{\Psi}$ is the set of vector corresponding to positive Hilbert-Schmidt operators. The inequality (5.10) correspond to the inequality $\|\sigma-\rho\|_{\mathrm{tr}} \geqq\left\|\sigma^{1 / 2}-\rho^{1 / 2}\right\|_{\mathrm{H} . \mathrm{S}}^{2}$ [7].

THEOREM 10 [6]. $\quad \tau_{\rho}(t) x \rightarrow \tau_{\psi}(t) x$ strongly as $\|\rho-\psi\| \rightarrow 0$ where $\rho$ and $\psi$ are faithful positive linear functionals of $R$, both $x \in R$ and $\psi$ are fixed.

Proof. Let $\xi_{\rho}=\sigma_{\psi}(\rho)$ and $\xi_{\psi}=\sigma_{\psi}(\psi)$ for some cyclic and separating $\Psi$. Then for $x \in R$,

$$
\begin{aligned}
& \left\|\Delta_{\xi \psi}^{1 / 2} x \xi_{\psi}-\Delta_{\xi}^{1 / 2} x \xi_{\rho}\right\|=\left\|J_{\Psi} \Delta_{\xi \psi}^{1 / 2} x \xi_{\psi}-J_{\Psi} \Delta_{\xi \rho}^{1 / 2} x \xi_{\rho}\right\| \\
& \quad=\left\|x^{*}\left(\xi_{\psi}-\xi_{\rho}\right)\right\| \leqq\|x\|\|\psi-\rho\|^{1 / 2}
\end{aligned}
$$

where we have used Theorem 4 (5) and (8). Hence

$$
\left\|\left(\Delta_{\xi \psi}^{1 / 2}+1\right) x \xi_{\psi}-\left(\Delta_{\xi_{\rho}}^{1 / 2}+1\right) x \xi_{\rho}\right\| \leqq 2\|x\|\|\psi-\rho\|^{1 / 2} .
$$

Since $\left\|\left(4_{\xi \psi}^{\mathrm{I} / 2}+1\right)^{-1}\right\| \leqq 1$, we have

$$
\begin{aligned}
& \left\|\left\{\left(\Delta_{\xi_{\rho}}^{1 / 2}+1\right)^{-1}-\left(\Delta_{\xi_{\psi}}^{1 / 2}+1\right)^{-1}\right\}\left(\Delta_{\xi_{\psi}}^{1 / 2}+1\right) x \xi_{\psi}\right\| \\
& \quad=\left\|\left(\Delta_{\xi_{\rho}}^{1 / 2}+1\right)^{-1}\left\{\left(\Delta_{\xi_{\psi}}^{1 / 2}+1\right) x \xi_{\psi}-\left(\Delta_{\xi_{\rho}}^{1 / 2}+1\right) x \xi_{\rho}\right\}+x\left(\xi_{\rho}-\xi_{\psi}\right)\right\| \\
& \quad \leqq 3\|x\|\|\psi-\rho\|^{1 / 2} .
\end{aligned}
$$

Since $\Delta_{\xi \psi}^{1 / 2}$ is essentially self-adjoint on $R \xi_{\psi},\left(\Delta_{\xi \psi}^{1 / 2}+1\right) R \xi_{\psi}$ is dense. 
Hence by uniform boundedness $\left\|\left(\Delta_{\xi_{\rho}}^{1 / 2}+1\right)^{-1}\right\| \leqq 1$,

$$
\left(\Delta_{\xi_{\rho}}^{1 / 2}+1\right)^{-1} \rightarrow\left(\Delta_{\xi_{\psi}}^{1 / 2}+1\right)^{-1}
$$

strongly as $\left\|\xi_{\rho}-\xi_{\psi}\right\| \rightarrow 0$. Let $f_{t}\left((u+1)^{-1}\right)=u^{2 i t}$. $f_{t}$ is a family of continuous functions on $(0,1)$, equicontinuous on compact subsets of $(0,1)$ for bounded $t$ and uniformly bounded. Hence by [4]

$$
\Delta_{\xi_{\rho}}^{i t} \rightarrow \Delta_{\xi \psi}^{i t} \text { strongly as }\|\rho-\psi\| \rightarrow 0
$$

uniformly in $t$ in a compact set. This implies $\tau_{\rho}(t) x \rightarrow \tau_{\psi}(t) x$ strongly as $\|\rho-\psi\| \rightarrow 0$, uniformly in $t$ in a compact set.

REMARK 3. A similar application yields an alternative proof of Theorem 3 of [6]:

In Theorem 3 of [6], let

$$
\varphi_{1}(x)=(1-\lambda)^{-1}\left\{\lambda \varphi\left(u x u^{*}\right)+(1-\lambda) \varphi\left(u u^{*} x u u^{*}\right)\right\} .
$$

Then $\varphi_{1} \geqq 0, \varphi_{1}$ is faithful if $\varphi$ is faithful and

$$
\begin{aligned}
\left\|\varphi_{1}(1)-1\right\| & =\lambda(1-\lambda)^{-1} \varphi\left(u u^{*}\right)-\varphi\left(u^{*} u\right) \\
& \leqq(1-\lambda)^{-1} \varepsilon
\end{aligned}
$$

We also have

$$
\begin{aligned}
|| \varphi_{1}(x)-\varphi(x) \| \leqq & (1-\lambda)^{-1}\left|\lambda \varphi\left(u x u^{*}\right)-(1-\lambda) \varphi\left(x u^{*} u\right)\right| \\
& +\lambda^{-1}\left|\lambda \varphi\left(u u^{*} x u u^{*}\right)-(1-\lambda) \varphi\left(u^{*} x u u^{*} u\right)\right| \\
& +\lambda^{-1}\left|(1-\lambda) \varphi\left(u^{*} x u\right)-\lambda \varphi\left(x u u^{*}\right)\right| \\
\leqq & (2-\lambda)(1-\lambda)^{-1} \lambda^{-1}\|x\| \varepsilon .
\end{aligned}
$$

Hence

$$
\left\|\varphi_{1}-\varphi\right\| \leqq(2-\lambda)(1-\lambda)^{-1} \lambda^{-1} \varepsilon .
$$

It is easily seen that $\lambda \varphi_{1}\left(x u^{*}\right)=(1-\lambda) \varphi\left(u^{*} x\right)$ and hence

$$
\left(\Delta_{\varphi_{1}}^{1 / 2}-\lambda^{1 / 2}(1-\lambda)^{-1 / 2}\right) u^{*} \xi_{\varphi_{1}}=0 \text {. }
$$

Since $\left\|u^{*} \xi_{\varphi_{1}}\right\|^{2}=\varphi\left(u u^{*}\right) \geqq 1-\lambda-\varepsilon$, we have

$$
\left\|\left(\Delta_{\varphi}^{1 / 2}-\lambda^{1 / 2}(1-\lambda)^{-1 / 2}\right) u^{*} \xi_{\varphi}\right\| \leqq\left(1+\lambda^{1 / 2}(1-\lambda)^{-1 / 2}\right)\left\|\varphi-\varphi_{1}\right\|^{1 / 2} .
$$

This proves Theorem 3 of [6].

Let $\operatorname{Aut}(R)$ denote the set of all *-automorphisms of R. Each $g \in \operatorname{Aut}(R)$ induces an adjoint mapping on $R_{*}^{+}$:

$$
\left(g^{*} \varphi\right)(x)=\varphi(g(x)) \text {. }
$$

THEOREM 11. There exists a unitary representation $U_{y}(g)$ of 
Aut $(R)$ such that

$$
\begin{array}{cc}
U_{\Psi}(g) x U_{\Psi}(g)^{*}=g(x), & x \in R, \\
U_{\Psi}(g) \sigma_{\Psi}\left(g^{*} \rho\right)=\sigma_{\Psi}(\rho), & \rho \in R_{*}^{+} .
\end{array}
$$

Each $U_{\psi}(g), g \in \operatorname{Aut}(R)$, commutes with $J_{\psi}$. For two cyclic and separating vectors $\Psi$ and $\Phi, U_{\Psi}$ and $U_{\Phi}$ are unitarily equivalent through a unitary operator $u^{\prime} \in R^{\prime}$ :

$$
u^{\prime} U_{y}(g)=U_{\Phi}(g) u^{\prime} .
$$

Proof. Let $\xi(g)=\sigma_{T}\left(g^{*} \omega_{\Psi}\right)$ where $\omega_{\Psi}$ is the expectation functional by $\Psi$. We define

$$
U_{0}(g) x \Psi=g(x) \xi\left(g^{-1}\right), \quad x \in R .
$$

We have

$$
\left(g(x) \xi\left(g^{-1}\right), g(y) \xi\left(g^{-1}\right)\right)=\left(g^{-1}\right)^{*} \omega_{Y}\left(g\left(x^{*} y\right)\right)=(x \Psi, y \Psi) .
$$

Hence $U_{0}(g)$ is well-defined and its closure $U_{y}(g)$ is isometric. Since $g^{*} \omega_{\Psi}$ is faithful because $\Psi$ is separating and $g$ is an automorphism, $\sigma_{\Psi}\left(g^{*} \omega_{\Psi}\right)=\xi(g)$ is separating. Since $\xi(g) \in V_{\Psi}$, it is cyclic if it is separating. Hence $U_{y}(g)$ is unitary.

From the definition (8.7), $U_{0}(g) x=g(x) U_{0}(g)$ and hence (8.4) holds.

Let $S_{1}=J_{\Psi} \Delta_{\Psi}^{1 / 2}$ and $S_{2}=J_{\xi} \Delta_{\xi}^{1 / 2}$ for $\xi=\xi\left(g^{-1}\right)$. We have

$$
\begin{aligned}
U(g) S_{1} x \Psi & =U(g) x^{*} \Psi=g\left(x^{*}\right) \xi \\
& =S_{2} g(x) \xi=S_{2} U(g) x \Psi
\end{aligned}
$$

for $x \in R$. Since $R \Psi$ is a core of $S_{1}$ and $R \xi$ is a core of $S_{2}$, we have $U(g) S_{1} U(g)^{*}=S_{2}$. By the uniqueness of polar decomposition, we have $U(g) J_{\Psi} U(g)^{*}=J_{\xi}$. Since $\xi\left(g^{-1}\right) \in V_{\Psi}$, we have $J_{\xi}=J_{\Psi}$. Hence $U(g)$ commutes with $J_{y}$.

Let $x \in R$ and $\psi \in V_{q}$. Then

$$
\begin{aligned}
\left(U(g) \psi, x j_{\xi}(x) \xi\right) & =\left(U(g) \psi,\left\{U(g) y U(g)^{*}\right\} J_{\xi}\left\{U(g) y U(g)^{*}\right\} \xi\right) \\
& =\left(\psi, y j_{\Psi}(y) \Psi\right) \geqq 0
\end{aligned}
$$

where $y=g^{-1}(x), J_{\xi}=J_{q}$, $\left[U(g), J_{\Psi}\right]=0, U(g)^{*} \xi=\Psi$. This implies

$$
U(g) \psi \in V_{\xi}^{\prime}=V_{\xi}=V_{\Psi} .
$$

Hence $U(g) V_{\Psi} \subset V_{\Psi}$.

By (8.4), we have for $\psi=U(g) \sigma_{\psi}(\rho)$ and $\rho \in R_{*}^{+}$

$$
\omega_{\psi}(g x)=\rho(x) .
$$

By $U(g) \sigma_{q}(\rho) \in V_{\Psi}$, we have (8.5). 
From (8.5), we have

$$
U_{\Psi}\left(g_{1}\right) U_{\Psi}\left(g_{2}\right) \psi=U_{\Psi}\left(g_{1} g_{2}\right) \psi
$$

for $\psi \in \sigma_{\Psi}\left(R_{*}^{+}\right)=V_{\Psi}$. Since $V_{\Psi}$ linearly span $H$, we have

$$
U_{\Psi}\left(g_{1}\right) U_{\Psi}\left(g_{2}\right)=U_{\Psi}\left(g_{1} g_{2}\right) \text {. }
$$

For two cyclic and separating vectors $\Psi$ and $\Phi$, there exists a unitary $u^{\prime} \in R^{\prime}$ such that $u^{\prime} \sigma_{\psi}\left(\omega_{\varphi}\right)=\Phi$, which automatically satisfies $u^{\prime} \sigma_{\Psi}(\rho)=\sigma_{\varphi}(\rho)$ for all $\rho \in R_{*}^{+}$. Then

$$
\begin{aligned}
u^{\prime} U_{\Psi}(g) \sigma_{\Psi}\left(g^{*} \rho\right) & =u^{\prime} \sigma_{\Psi}(\rho)=\sigma_{\varphi}(\rho)=U_{\varphi}(g) \sigma_{\varphi}\left(g^{*} \rho\right) \\
& =U_{\varphi}(g) u^{\prime} \sigma_{\Psi}\left(g^{*} \rho\right) .
\end{aligned}
$$

Since $\sigma_{y}\left(g^{*} \rho\right), \rho \in R_{*}^{+}$, is total, we have (8.6).

REMARK. The weak, strong and *-strong topologies coincide on unitaries and they induce a topology $\tau_{U}$ on Aut $(R)$ through $U_{y}(g)$. Since the multiplication of unitaries is continuous relative to strong topology, (Aut $\left.(R), \tau_{U}\right)$ is a topological group. On Aut $(R)$ there is a topology $\tau$ by the norm convergence of $g^{*} \rho$ for every $\rho \in R_{*}^{+}$. The two topologies $\tau$ and $\tau_{U}$ coincide which can be seen as follows:

The strong convergence of $U_{\Psi}(g)$ is equivalent to the strong convergence of $U_{w}(g)^{*}$.

Since $V_{y}$ span $H$, the strong convergence of $U_{\Psi}(g)^{*}$ is equivalent to the strong convergence of $U_{\Psi}\left(g^{-1}\right) \sigma_{\Psi}(\rho)=\sigma_{\Psi}\left(g^{*} \rho\right)$ for each $\rho \in R_{*}^{+}$.

Since $\sigma_{T}$ is a homeomorphism, the strong convergence of $\sigma_{\Psi}\left(g^{*} \rho\right)$ is equivalent to the norm convergence of $g^{*} \rho$ for each $\rho \in R_{*}^{+}$.

9. Radon-Nikodym derivative satisfying a chain rule.

THEOREM 12. Let $\rho, \mu \in R_{*}^{+}$and $\Psi$ be a cyclic and separating vector.

(1) The following two conditions are equivalent.

( $\alpha) \quad l \rho \geqq \mu$ for some $l$.

( $\beta)$ There exists $A=A(\mu / \rho) \in R$ such that

$$
\begin{gathered}
\mu(x)=\rho\left(A^{*} x A\right), \quad A \sigma_{\Psi}(\rho)=\sigma_{\Psi}(\mu), \\
s(\rho) \geqq s\left(A^{*} A\right) .
\end{gathered}
$$

The operator $A \in R$ satisfying (9.1) and (9.2) is unique.

(2) If $(\alpha)$ or $(\beta)$ holds, then

$$
\begin{gathered}
\|A(\mu / \rho)\|^{2}=\inf \{l ; l \rho \geqq \mu\}, \\
\|A(\mu / \rho)\| \sigma_{\Psi}(\rho) \geqq \sigma_{\Psi}(\mu)
\end{gathered}
$$


where $x \geqq y$ denotes $x-y \in V_{\Psi}$.

(3) If $l_{1} \mu_{1} \geqq \mu_{2}, l_{2} \mu_{2} \geqq \mu_{3}$, then

$$
A\left(\mu_{3} / \mu_{1}\right)=A\left(\mu_{3} / \mu_{2}\right) A\left(\mu_{2} / \mu_{1}\right) .
$$

(4) $A(\mu / \mu)=s(\mu)$.

(5) $A(\mu / \rho)$ does not depend on $\Psi$.

Proof. (1) First assume ( $\beta)$. Noting $J_{y} \sigma_{\psi}(\rho)=\sigma_{y}(\rho)$, we have

$$
\sigma_{\psi}(\mu)=J_{\psi} \sigma_{\psi}(\mu)=J_{\Psi} A \sigma_{\psi}(\rho)=j_{\Psi}(A) \sigma_{\psi}(\rho) .
$$

Hence

$$
\begin{aligned}
\mu(Q) & =\left(Q^{1 / 2} \sigma_{\psi}(\rho), j_{\Psi}\left(A^{*} A\right) Q^{1 / 2} \sigma_{\psi}(\rho)\right) \\
& \leqq\left\|j_{\Psi}\left(A^{*} A\right)\right\| \rho(Q)
\end{aligned}
$$

for $Q \geqq 0, Q \in R$. Hence $(\beta)$ implies $(\alpha)$.

Next assume $(\alpha)$. Then there exists $t^{\prime} \in R^{\prime}$ such that

$$
\sigma_{\varphi}(\mu)=t^{\prime} \sigma_{\Psi}(\rho), \quad\left\|t^{\prime}\right\| \leqq l .
$$

Since $J_{\Psi} \sigma_{\Psi}(\mu)=\sigma_{\Psi}(\mu)$ and $J_{y} \sigma_{\Psi}(\rho)=\sigma_{\Psi}(\rho)$, we have

$$
\begin{aligned}
\sigma_{\Psi}(\mu)=J_{y} \sigma_{\Psi}(\mu) & =J_{\Psi}\left\{t^{\prime} s^{R^{\prime}}\left(\sigma_{\Psi}(\rho)\right)\right\} \sigma_{\Psi}(\rho) \\
& =j_{\Psi}\left\{t^{\prime} s^{R^{\prime}}\left(\sigma_{\Psi}(\rho)\right)\right\} \sigma_{\Psi}(\rho) .
\end{aligned}
$$

Hence we have (9.1) with

$$
A=j_{\Psi}\left\{t^{\prime} s^{R^{\prime}}\left(\sigma_{\Psi}(\rho)\right)\right\} .
$$

Since $j_{\Psi}\left\{s^{R^{\prime}}\left(\sigma_{\Psi}(\rho)\right)\right\}=s^{R}\left(\sigma_{\Psi}(\rho)\right)=s(\rho)$ due to $J_{\Psi} \sigma_{\Psi}(\rho)=\sigma_{\Psi}(\rho)$, we have

$$
s^{R}\left(A^{*} A\right) \leqq j_{\Psi}\left\{s^{R^{\prime}}\left(\sigma_{\Psi}(\rho)\right)\right\}=s(\rho) .
$$

If $A_{1} \sigma_{\Psi}(\rho)=A_{2} \sigma_{\Psi}(\rho)=\sigma_{\Psi}(\mu)$, then $\left(A_{1}-A_{2}\right) \sigma_{\Psi}(\rho)=0$. Hence $\left(A_{1}-A_{2}\right) s^{R}\left(\sigma_{r}(\rho)\right)=0$. By $(9.2), A_{k} s^{R}\left(\sigma_{\Psi}(\rho)\right)=A_{k}$ and hence $A_{1}=A_{2}$.

(2) From (9.6), we have

$$
l_{0} \equiv \inf \{l ; l \rho \geqq \mu\} \leqq\left\|A^{*} A\right\|=\|A\|^{2} .
$$

From (9.7), we have

$$
\|A\|^{2} \leqq\left\|t^{\prime}\right\|^{2} \leqq l
$$

for any $l$ satisfying $l \rho \geqq \mu$. Hence we have (9.3).

To prove (9.4), we first show that

$$
s\left(A A^{*}\right)=s(\mu) .
$$

For $e \in R, e \geqq 0, \mu(e)=0$ is equivalent to $e A \sigma_{\Psi}(\rho)=0$, which is equiva- 
lent to $e A=0$ due to $s\left(A^{*} A\right) \leqq s(\rho)$. Hence (9.8) holds. We now consider restriction of $R$ and $H$ by $s(\rho) j_{\Psi}\{s(\rho)\}$. Let $M=s(\rho) R s(\rho) \mid K$, $K=s(\rho) j_{w}\{s(\rho)\} H . \quad \xi_{\rho}$ is cyclic and separating and

$$
s(\rho) j_{\Psi}\{s(\rho)\} A \xi_{\rho}=s(\rho) A \xi_{\rho}=A \xi_{\rho}
$$

where $s(\rho) \geqq s(\mu)$ due to $l \rho \geqq \mu$, which implies $s(\rho) A=A$, and $j_{\Psi}\{s(\rho)\} \xi_{\rho}=\xi_{\rho}$. Thus $A \xi_{\rho} \in V_{\xi_{\rho}}=s(\rho) j_{\Psi}\{s(\rho)\} V_{\Psi}$.

By Theorem 3 (9), we have (9.4).

(3) follows from the uniqueness.

(4) $s(\mu)$ satisfies (9.1) and (9.2) with $\rho=\mu$.

(5) follows from Theorem 7 (6).

Remark. If $R$ is commutative, $A(\mu / \rho)$ is the same as the positive square root of the Radon-Nikodym derivative in measure theoretical sense. The following theorem gives a condition that $A(\mu / \rho)$ coincides with Sakai's noncommutative Radon-Nikodym derivative. Because of the chain rule, it also coincides with the condition $A_{1}(\mu / \rho)=A_{2}(\mu / \rho)$ when $l_{1} \mu \geqq \rho$ and $l_{2} \rho \geqq \mu$, where $A_{k}(\mu / \nu), k=1,2$, are defined in [3].

THEOREM 13. If $l \rho \geqq \mu$, the following conditions are equivalent.

(a) $A(\mu / \rho)^{*}=A(\mu / \rho)$,

(b) $A(\mu / \rho) \geqq 0$,

(c) $\tau_{\rho}(t) A(\mu / \rho)=A(\mu / \rho)$ where $\tau_{\rho}(t)$ is the modular automorphism for the state $\rho$ of the reduced algebra $s(\rho) R s(\rho)$.

(d) $\mu$ commutes with $\rho$.

Proof. If (c) holds, then $A(\mu / \rho) \xi_{\rho}=\xi_{\mu} \in V_{\xi_{\rho}}$ implies

$$
0 \leqq \tau_{\rho}(i / 4) A(\mu / \rho)=A(\mu / \rho) .
$$

Hence (c) implies (b). (b) trivially implies (a).

Assume (a). For any $Q \in R$ and $A=A(\mu / \rho)$, we have

$$
\begin{aligned}
\left(\xi_{\rho}, Q A \xi_{\rho}\right) & =\left(\xi_{\rho}, Q J_{\xi_{\rho}} A \xi_{\rho}\right)=\left(\xi_{\rho}, Q j_{\xi_{\rho}}(A) \xi_{\rho}\right) \\
& =\left(j_{\xi_{\rho}}(A) \xi_{\rho}, Q \xi_{\rho}\right)=\left(J_{\xi_{\rho}} A \xi_{\rho}, Q \xi_{\rho}\right) \\
& =\left(A \xi_{\rho}, Q \xi_{\rho}\right)=\left(\xi_{\rho}, A Q \xi_{\rho}\right) .
\end{aligned}
$$

Such $A$ is known to be invariant under $\tau_{\rho}(t)$. ([9])

The equivalence of (c) and (d) is known. ([9])

10. $\Psi$-bounded operators. We shall call $Q \in R \Psi$-bounded if

$$
\omega_{Q \Psi} \leqq l \omega_{\Psi} .
$$

for some $l \geqq 0$. We shall call $Q \in R \Psi$-symmetric if 


$$
J_{\Psi} Q \Psi=Q \Psi .
$$

We shall call $Q \in R \Psi$-positive if

$$
Q \Psi \in V_{\Psi} .
$$

THEOREM 14. that

(1) $Q$ is $\Psi$-bounded if and only if there exists $a Q^{\Psi} \in R$ such

$$
\Delta_{\Psi}^{1 / 2} Q^{*} \Psi=Q^{\Psi} \Psi
$$

(2) Any $\Psi$-bounded $Q$ can be decomposed as $Q=Q_{r}+i Q_{i}$ where $Q_{r}, Q_{i} \in R$ and both are $\Psi$-symmetric.

(3) Any $\Psi$-symmetric $Q \in R$ is $\Psi$-bounded and $Q^{\Psi}=Q$. It has a decomposition

$$
Q=Q_{1}-Q_{2}
$$

where $Q_{1}, Q_{2} \in R$, both $Q_{1}$ and $Q_{2}$ are $\Psi$-positive, $\left\|Q_{1}\right\| \leqq\|Q\|,\left\|Q_{2}\right\| \leqq\|Q\|$ and

$$
s\left(Q_{1} Q_{1}^{*}\right) \perp s\left(Q_{2} Q_{2}^{*}\right) .
$$

(4) Any $Q \in R$ has a unique decomposition

$$
Q=u|Q|_{T}
$$

where $u$ is a partial isometry in $R$ such that

$$
u^{*} u=s\left(|Q|_{x}|Q|_{*}^{*}\right)
$$

and $|Q|_{\Psi}$ is $\Psi$-positive.

(5) $Q \in R$ is $\Psi$-positive if and only if $Q$ is $\Psi$-symmetric and $\tau_{y}(i / 4) Q$ is positive.

Proof (1). If $\omega_{Q \Psi} \leqq l \omega_{\Psi}$, then there exists $Q^{\prime} \in R^{\prime}, 0 \leqq Q^{\prime} \leqq l^{1 / 2}$ such that $\omega_{Q T}=\omega_{Q^{\prime} \Psi}$. Then there exists a partial isometry $u^{\prime} \in R^{\prime}$ such that $Q \Psi=u^{\prime} Q^{\prime} \Psi$. Let $Q^{\Psi} \equiv j_{\Psi}\left(u^{\prime} Q^{\prime}\right)$. We have

$$
Q^{\Psi} \Psi=J_{\Psi} u^{\prime} Q^{\prime} \Psi=J_{\Psi} Q \Psi=\Delta_{\Psi}^{1 / 2} Q^{*} \Psi .
$$

Conversely, if (10.1) holds, then

$$
Q \Psi=J_{\Psi} \Delta_{\Psi}^{1 / 2} Q^{*} \Psi=J_{\Psi} Q^{\Psi} \Psi=j_{\Psi}\left(Q^{\Psi}\right) \Psi .
$$

Hence $\omega_{Q \Psi} \leqq\left\|j_{\Psi}\left(Q^{\Psi}\right)\right\|^{2} \omega_{\Psi}$.

(2) Define $Q_{r}=\left(Q+Q^{\Psi}\right) / 2, Q_{i}=\left(Q-Q^{\Psi}\right) /(2 i)$. Then both are $\Psi$-symmetric and $Q=Q_{r}+i Q_{i}$.

(3) Let $Q \Psi=\Phi_{1}-\Phi_{2}, \Phi_{1} \in V_{\Psi}, \Phi_{2} \in V_{\Psi}, s^{R^{\prime}}\left(\Phi_{1}\right) \perp s^{R^{\prime}}\left(\Phi_{2}\right), s^{R}\left(\Phi_{1}\right) \perp$ 
$S^{R}\left(\Phi_{2}\right)$ be the decomposition given by Theorem 4 (6). Denote $s^{\prime}=s^{R^{\prime}}\left(\Phi_{1}\right)$. We have $\Phi_{1}=s^{\prime} Q \Psi$. Hence $\omega_{\Phi_{1}}=w_{s^{\prime} Q \Psi} \leqq \omega_{Q \Psi}$. Since $Q \Psi=J_{\psi} Q \Psi=$ $j_{\Psi}(Q) \Psi, \omega_{Q \Psi} \leqq\left\|j_{\Psi}(Q)\right\|^{2} \omega_{\psi}=\|Q\|^{2} \omega_{\psi}$. Hence by Theorem $3(8)$, there exists a $\Psi$-positive $Q_{1} \in R$ such that $\Phi_{1}=Q_{1} \Psi$ and $\left\|Q_{1}\right\| \leqq\|Q\|$. Similarly there exists $\Psi$-positive $Q_{2} \in R$ such that $\Phi_{2}=Q_{2} \Psi$ and $\left\|Q_{2}\right\| \leqq\|Q\|$. Since $\Psi$ is separating, (10.2) holds.

Since $\Psi$ is separating for $R$, we have $s^{R}\left(Q_{k} \Psi\right)=s\left(Q_{k} Q_{k}^{*}\right), k=1,2$. Since $s^{R}\left(\Phi_{1}\right) \perp s^{R}\left(\Phi_{2}\right)$, we have (10.3).

(4) Let $\rho=\omega_{J \Psi Q \Psi}$. Then $\rho \leqq\left\|j_{\Psi}(Q)\right\|^{2} \omega_{\Psi}=\|Q\|^{2} \omega_{\Psi}$. Hence there exists a $\Psi$-positive $Q_{1} \in R$ such that $\sigma_{\Psi} \rho=Q_{1} \Psi$. Since $\omega_{J_{\Psi Q \Psi}}=$ $\omega_{Q_{1} \Psi}$, there exists a partial isometry $u^{\prime} \in R^{\prime}$ such that $J_{\Psi} Q \Psi=u^{\prime} Q_{1} \Psi$ and $u^{\prime *} u^{\prime}=s^{R^{\prime}}\left(Q_{1} \Psi\right)=j_{\Psi}\left\{s^{R}\left(Q_{1} \Psi\right)\right\}=j_{\Psi}\left\{s\left(Q_{1} Q_{1}^{*}\right)\right\}$ where we have used the property $J_{\Psi} Q_{1} \Psi=Q_{1} \Psi$.

We now have $Q \Psi=J_{\Psi} u^{\prime} Q_{1} \Psi=j_{\Psi}\left(u^{\prime}\right) J_{\Psi} Q_{1} \Psi=u Q_{1} \Psi$ where $u \equiv j_{y}\left(u^{\prime}\right)$. Since $\Psi$ is separating for $R, Q=u Q_{1}$. We have $u^{*} u=j_{\Psi}\left(u^{\prime *} u^{\prime}\right)=$ $s\left(Q_{1} Q_{1}^{*}\right)$. Hence $Q_{1}=|Q|_{\Psi}$ and $u$ satisfy (10.4) and (10.5).

Conversely, assume that $Q=u_{k} Q_{k}, Q_{k}$ is $\Psi$-positive, $u_{k}$ is partially isometric, $u_{k}, Q_{k} \in R, u_{k}^{*} u_{k}=s\left(Q_{k} Q_{k}^{*}\right), k=1,2$. Then $\omega_{J \Psi Q \Psi}=\omega_{Q_{k} \Psi}$ where we have used $J_{\psi} Q_{k} \Psi=Q_{k} \Psi$. Since $Q_{k} \Psi \in V_{\Psi}$, such $Q_{k} \Psi$ is unique by Theorem 7 (1) and we have $Q_{1}=Q_{2}$.

Since $u_{1} Q_{1}=u_{2} Q_{2}=u_{2} Q_{1}$, we have $\left(u_{1}-u_{2}\right) s\left(Q_{1} Q_{1}^{*}\right)=0$. Since $u_{1}^{*} u_{1}=s\left(Q_{1} Q_{1}^{*}\right)=s\left(Q_{2} Q_{2}^{*}\right)=u_{2}^{*} u_{2}$, we have $u_{k} s\left(Q_{1} Q_{1}^{*}\right)=u_{k}, k=1,2$, and hence $u_{1}=u_{2}$.

(5) $Q$ is $\Psi$-symmetric if $Q$ is $\Psi$-positive by (5.2). By Theorem 3 (7) with $\alpha=1 / 4, \tau_{\Psi}(i / 4) Q \geqq 0$ if $Q \Psi \in V_{\Psi}$. If $Q$ is $\Psi$-symmetric, then $J Q \Psi=Q \Psi$. Hence $\Delta_{\Psi}^{1 / 2} Q^{*} \Psi=Q \Psi$, which implies $\Delta_{\Psi}^{-1 / 2} Q \Psi=Q^{*} \Psi$. Hence $\tau_{\Psi(z)} Q \in R$ can be defined by Lemma 6 for $\operatorname{Im} z \in[0,1 / 2]$. Hence $\left(\Delta_{\Psi}^{-1 / 4} Q \Delta_{\psi}^{1 / 4}\right)^{-}$ is in $R$. If it is positive, then $Q \Psi \in V_{\Psi}$ by Theorem 3 (7).

THEOREM 15. If $\rho \leqq l \omega_{\Psi}$, there exists $Q \in R, 0 \leqq Q \leqq l^{1 / 4}$ such that $\sigma_{\Psi} \rho=Q j_{\Psi}(Q) \Psi$.

Proof. Let $\rho_{1}(A)=\left(\sigma_{\Psi} \rho, \Delta_{\Psi}^{1 / 4} A \Psi\right)$ for $A \in R$. Then $\rho_{1} \in R_{*}^{+}$. Since $\rho \leqq l \omega_{\psi}$, there exists $Q_{1} \in R$ such that $Q_{1} \Psi=\sigma_{\Psi} \rho,\left\|Q_{1}\right\|^{2} \leqq l$. Then $Q_{1} \Psi=j_{\Psi}\left(Q_{1}\right) \Psi$ and

$$
\Delta_{\Psi}^{1 / 4} j_{\Psi}\left(Q_{1}\right) \Psi=j_{\Psi}\left(\tau_{\Psi}(i / 4) Q_{1}\right) \Psi,
$$

where

$$
0 \leqq \tau_{\psi}(i / 4) Q_{1} \equiv Q_{2} \leqq\left\|Q_{1}\right\| \leqq l^{1 / 2}
$$

by Theorem 3 (7).

Set $Q_{2}^{\prime}=j_{\Psi}\left(Q_{2}\right)$. We have

$$
\rho_{1}(A)=\left(Q_{2}^{\prime} \Psi, A \Psi\right)=\left(Q_{2}^{\prime 1 / 2} \Psi, A Q_{2}^{\prime 1 / 2} \Psi\right) .
$$


Hence $\rho_{1} \leqq\left\|Q_{2}^{\prime}\right\| \omega_{\Psi} \leqq l^{1 / 2} \omega_{\psi}$. By Theorem 7 (1), there exists a $\Psi$-positive $Q_{3} \in R$ such that $\sigma_{\Psi} \rho_{1}=Q_{3} \Psi,\left\|Q_{3}\right\| \leqq l^{1 / 4}$. Let $Q=\tau_{\Psi}(i / 4) Q_{3}$. By Theorem $3(7),\|Q\| \leqq\left\|Q_{3}\right\| \leqq l^{1 / 4}$ and $Q \geqq 0$. We have $Q_{3} \Psi=J_{\Psi} Q_{3} \Psi=$ $j_{Y}\left(Q_{3}\right) \Psi$ and hence

$$
\begin{aligned}
& \left(\sigma_{\Psi} \rho, \Delta_{\Psi}^{1 / 4} A \Psi\right)=\left(Q_{3} \Psi, A Q_{3} \Psi\right)=\left(Q_{3} \Psi, A j_{\Psi}\left(Q_{3}\right) \Psi\right) \\
& \quad=\left(Q_{3} j_{\Psi}\left(Q_{3}^{*}\right) \Psi, A \Psi\right)=\left(\left\{\tau_{\Psi}(i / 4) Q_{3}\right\}_{\Psi}\left(\left\{\tau_{\Psi}(i / 4) Q_{3}\right\}^{*}\right) \Psi, \Delta_{\Psi}^{1 / 4} A \Psi\right)
\end{aligned}
$$

Since $\triangle_{\Psi}^{1 / 4} A \Psi, A \in R$, is dense, we have

$$
\sigma_{\Psi} \rho=Q j_{\Psi}(Q) \Psi .
$$

11. Additional remarks. In this paper, we have assumed that $R$ has a faithful normal state. This assumption is not essential in defining $d^{\prime}\left(\rho_{1}, \rho_{2}\right)$ and $\left(\sigma_{\Psi} \rho_{1}, \sigma_{\Psi} \rho_{2}\right)$. They can be defined relative to $s R s$ where $s=s\left(\rho_{1}\right) \vee s\left(\rho_{2}\right)$. With such definition, Theorem 9 holds.

The cone $W_{\Psi}$ has been introduced as the weakly closed convex hull of $Q j_{y}(Q), Q \in R$. It is a weakly closed selfadjoint convex cone which form a semigroup under multiplication. It is total in $W \equiv\left(R \cup R^{\prime}\right)^{\prime \prime}$.

If $\rho \in W_{*}$ is of the form $\rho=\sum_{j} \omega_{x_{j} y_{j}}$ with $x_{j}, y_{j} \in V_{\psi}$, then $\rho(w) \geqq 0$ for all $w \in W_{\Psi}$. If $\rho \in W_{*}, \rho=\omega_{x}$ and $\rho(w) \geqq 0$ for all $w \in W_{\Psi}$, then $\rho=\omega_{y}$ for $y \in V_{\Psi}$ by Theorem 3. It is of interest to determine the dual of $W_{\Psi}$ in $W_{*}$. If $R$ is a type $I$ factor, the dual of $W_{\Psi}$ consists of $\rho=\sum_{j} \omega_{x_{j} y_{j}}, x_{j}, y_{j} \in V_{\Psi}$.

Acknowledgment. The author would like to thank Professor Coleman and Professor Woods for their warm hospitality at Department of Mathematics, Queen's University, where this work has been done. The author would like to thank Drs. G. Elliott and O. Nielsen for helpful comments.

Appendix. The result that $V_{\Psi}$ is selfdual can be proved directly as follows:

We define $V_{\Psi}$ first as the closed convex hull of $\left\{Q j_{\Psi}(Q) \Psi ; Q \in R\right\}$. Then (5.1) (5.4) are immediate. In particular (5.4) shows $V_{T} \subset V_{\Psi}^{\prime}$. Let $\Phi \in V_{\Psi}^{\prime}$.

By noncommutative Radon-Nikodym theorem, there exists a positive selfadjoint $A_{2}$ affiliated with $R$ and a partial isometry $u^{\prime} \in R^{\prime}$ such that $\Phi=u^{\prime} A_{2} \Psi$. If $A_{2}=\int \lambda d E_{\lambda}$, we set $A_{2}^{L}=A_{2} E_{L}$ and

$$
\Phi^{L} \equiv E_{L} j_{Y}\left(E_{L}\right) \Phi=j_{\Psi}\left(E_{L}\right) u^{\prime} A_{2}^{L} \Psi .
$$

Then $\lim \Phi^{L}=\Phi$ and $\Phi^{L} \in V_{\Psi}^{\prime}$. Since $\omega_{\Phi} L \leqq \omega_{A_{2}^{L}}^{L}$, there exists $t \in R$, $0 \leqq t \leqq 1$ and a partial isometry $w \in R^{\prime}$ such that

$$
\Phi^{L}=w t A_{2}^{L} \Psi, w^{*} \Phi^{L}=t A_{2}^{L} \Psi, s^{R^{\prime}}\left(\Phi^{L}\right)=w w^{*} .
$$


Set $\Phi^{\prime}=A_{3} \Psi, A_{3} \equiv j_{\Psi}\left(w^{*}\right) t A_{2}^{L} \in R$. Then $\Phi^{\prime}=w^{*} j_{\Psi}\left(w^{*}\right) \Phi^{L} \in V_{\Psi}^{\prime}$. Since $\Phi^{L} \in V_{\Psi}^{\prime}$, we have $\left(x, J_{\Psi} \Phi^{L}\right)=\left(\Phi^{L}, J_{\Psi} x\right)=\left(\Phi^{L}, x\right)=\left(x, \Phi^{L}\right) \geqq 0$ for $x \in V_{\Psi}$. Since $V_{\Psi}$ is total, we have $J_{y} \Phi^{L}=\Phi^{L}$ and hence $j_{\Psi}(w) w \Phi^{\prime}=$ $j_{\Psi}\left(w w^{*}\right) \Phi^{L}=\Phi^{L}$. Hence it is enough to show $\Phi^{\prime} \in V_{\Psi}$. Let $\Phi_{\beta}^{\prime}=A_{4} \Psi$, $A_{4}=A_{3}\left(f_{\beta}^{G}\right)$ defined by (3.7) and (3.11). Then $\Phi_{\beta}^{\prime} \in V_{\Psi}^{\prime}$ and $\lim _{\beta \rightarrow+0} \Phi_{\beta}^{\prime}=\Phi^{\prime}$.

Let $A_{5}=\tau(i / 4) A_{4}$. Since $A_{4} \Psi \in V_{\Psi}^{\prime}$, we have

$$
\begin{aligned}
\left(\Delta_{\Psi}^{1 / 4} \Phi \Psi, A_{5} \Delta_{\Psi}^{1 / 4} Q \Psi\right) & =\left(Q \Psi, A_{4} \Delta_{\Psi}^{1 / 2} Q \Psi\right)=\left(Q \Psi, A_{4} j_{\Psi}\left(Q^{*}\right) \Psi\right) \\
& =\left(j_{\Psi}(Q) Q \Psi, A_{4} \Psi\right) \geqq 0 .
\end{aligned}
$$

Since $\Delta_{\psi}^{1 / 4} R \Psi$ is dense, we have $A_{5} \geqq 0$. Let $B=A_{5}^{1 / 2}, B_{\gamma}=B\left(f_{\gamma}^{G}\right)$. Then $\lim B_{r}^{2}=A_{5}$ and

$$
\begin{aligned}
\left\|\Delta_{\Psi}^{1 / 4}\left(B_{\gamma}^{2}-A_{5}\right) \Psi\right\|^{2} & =\left(\left\{B_{\gamma}^{2}-A_{5}\right\} \Psi, \Delta_{\Psi}^{1 / 2}\left\{B_{\gamma}^{2}-A_{5}\right\} \Psi\right) \\
& =\left(\left\{B_{\gamma}^{2}-A_{5}\right\} \Psi, J_{\Psi}\left\{B_{\gamma}^{2}-A_{5}\right\} \Psi\right) \rightarrow 0
\end{aligned}
$$

as $\gamma \rightarrow+0$. Therefore,

$$
\lim \Delta_{\Psi}^{1 / 4} B_{\gamma}^{2} \Psi=\Delta_{\Psi}^{1 / 4} A_{5} \Psi=\left\{\tau_{\Psi}(-i / 4) A_{5}\right\} \Psi=A_{4} \Psi=\Phi_{\beta}^{\prime} .
$$

We also have

$$
\Delta_{\Psi}^{1 / 4} B_{r}^{2} \Psi=C j_{\Psi}(C) \Psi
$$

for $C=\tau_{\Psi}(-i / 4) B_{\gamma}$ due to $J_{\Psi} C \Psi=C \Psi$. Hence $\Delta_{\Psi}^{1 / 4} B_{\gamma}^{2} \Psi \in V_{\Psi}$. This completes the proof.

\section{REFERENCES}

1. H. Araki, Multiple time analyticity of a quantum statistical state satisfying the KMS boundary condition, Publ. RIMS, 4A (1968), 361-371.

2. - On quasifree states of CAR and Bogolubov automorphisms, Publ. RIMS, 6 (1970/71), 385-442.

3. - Bures distance function and a generalization of Sakai's noncommutative Radon-Nikodym theorem, Publ. RIMS, 8 (1972/73), 335-362.

4. H. Araki and E. J. Woods, Topologies induced by representations of the canonical commutation relations, Rep. Math. Phys., 4 (1973), 227-254.

5. D. J. C. Bures, An extension of Kakutani's theorem on infinite product measures to the tensor product of semifinite $W^{*}$-algebras, Trans. Amer. Math. Soc., 135 (1969), 199-212.

6. A. Connes, États presque périodiques sur une algèbre de von Neumann, C. R. Acad. Sc. Paris, 274 (1972), 1402-1405.

7. R. T. Powers and E. Størmer, Free states of the canonical anticommutation relations, Commun. Math. Phys., 16 (1970), 1-33.

8. S. Sakai, $C^{*}$-Algebras and $W^{*}$-Algebras, Springer Verlag, New York-Heidelberg-Berlin, 1971.

9. M. Takesaki, Tomita's Theory of Modular Hilbert Algebras and its Applications, Springer Verlag, Berlin-Heidelberg-New York, 1970.

Received September 29, 1972.

KYOTO UNIVERSITY 


\section{PACIFIC JOURNAL OF MATHEMATICS}

\section{EDITORS}

RICHARD ARENS (Managing Editor)

University of California

Los Angeles, California 90024

\section{R. A. Beaumont \\ University of Washington \\ Seattle, Washington 98105}

\section{J. DugundjI*}

Department of Mathematics University of Southern California Los Angeles, California 90007

D. Gilbarg and J. Milgram

Stanford University

Stanford, California 94305

\section{ASSOCIATE EDITORS}
E. F. BeCKenBaCH
B. H. NeumanN
F. WOLF
K. YoSHIDA

\section{SUPPORTING INSTITUTIONS}

\author{
UNIVERSITY OF BRITISH COLUMBIA \\ CALIFORNIA INSTITUTE OF TECHNOLOGY \\ UNIVERSITY OF CALIFORNIA \\ MONTANA STATE UNIVERSITY \\ UNIVERSITY OF NEVADA \\ NEW MEXICO STATE UNIVERSITY \\ OREGON STATE UNIVERSITY \\ UNIVERSITY OF OREGON \\ OSAKA UNIVERSITY
}

\author{
UNIVERSITY OF SOUTHERN CALIFORNIA \\ STANFORD UNIVERSITY \\ UNIVERSITY OF TOKYO \\ UNIVERSITY OF UTAH \\ WASHINGTON STATE UNIVERSITY \\ UNIVERSITY OF WASHINGTON

$* * *$
$*$
AMERICAN MATHEMATICAL SOCIETY
NAVAL WEAPONS CENTER

The Supporting Institutions listed above contribute to the cost of publication of this Journal, but they are not owners or publishers and have no responsibility for its content or policies.

Mathematical papers intended for publication in the Pacific Journal of Mathematics should be in typed form or offset-reproduced, (not dittoed), double spaced with large margins. Underline Greek letters in red, German in green, and script in blue. The first paragraph or two must be capable of being used separately as a synopsis of the entire paper. Items of the bibliography should not be cited there unless absolutely necessary, in which case they must be identified by author and Journal, rather than by item number. Manuscripts, in duplicate if possible, may be sent to any one of the four editors. Please classify according to the scheme of Math. Rev. Index to Vol. 39. All other communications to the editors should be addressed to the managing editor, or Elaine Barth, University of California, Los Angeles, California, 90024.

100 reprints are provided free for each article, only if page charges have been substantially paid. Additional copies may be obtained at cost in multiples of 50 .

The Pacific Journal of Mathematics is issued monthly as of January 1966. Regular subscription rate: $\$ 60.00$ a year (6 Vols., 12 issues). Special rate: $\$ 30.00$ a year to individual members of supporting institutions.

Subscriptions, orders for back numbers, and changes of address should be sent to Pacific Journal of Mathematics, 103 Highland Boulevard, Berkeley, California, 94708.

PUBLISHED BY PACIFIC JOURNAL OF MATHEMATICS, A NON-PROFIT CORPORATION

Printed at Kokusai Bunken Insatsusha (International Academic Printing Co., Ltd.), 270, 3-chome Totsuka-cho, Shinjuku-ku, Tokyo 160, Japan

* C. R. DePrima California Institute of Technology, Pasadena, CA 91109, will replace J. Dugundji until August 1974. 


\section{Pacific Journal of Mathematics}

Vol. 50, No. $2 \quad$ October, 1974

Mustafa Agah Akcoglu, John Philip Huneke and Hermann Rost, A counter example to the Blum Hanson theorem in general spaces .............

Huzihiro Araki, Some properties of modular conjugation operator of von

Neumann algebras and a non-commutative Radon-Nikodym theorem

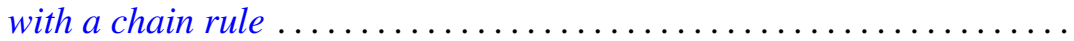

E. F. Beckenbach, Fook H. Eng and Richard Edward Tafel, Global properties of rational and logarithmico-rational minimal surfaces .....

David W. Boyd, A new class of infinite sphere packings ............. 383

K. G. Choo, Whitehead Groups of twisted free associative algebras ........

Charles Kam-Tai Chui and Milton N. Parnes, Limit sets of power series

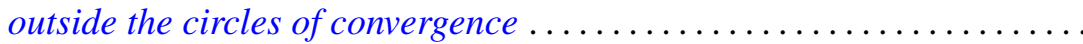

Allan Clark and John Harwood Ewing, The realization of polynomial

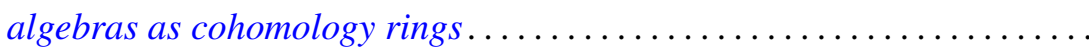

Dennis Garbanati, Classes of circulants over the p-adic and rational

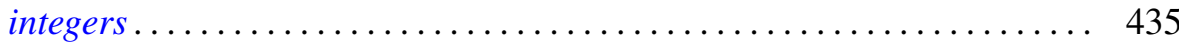

Arjun K. Gupta, On a "square" functional equation ................... 449

David James Hallenbeck and Thomas Harold MacGregor, Subordination and extreme-point theory ............................. 455

Douglas Harris, The local compactness of $v X \ldots \ldots . . . . . . . . . . . .4469$

William Emery Haver, Monotone mappings of a two-disk onto itself which fix the disk's boundary can be canonically approximated by homeomorphisms .................................. 477

Norman Peter Herzberg, On a problem of Hurwitz .................. 485

Chin-Shui Hsu, A class of Abelian groups closed under direct limits and subgroups formation ............................... 495

Bjarni Jónsson and Thomas Paul Whaley, Congruence relations and multiplicity types of algebras.....................

Lowell Duane Loveland, Vertically countable spheres and their wild sets.

Nimrod Megiddo, Kernels of compound games with simple components ....

Russell L. Merris, An identity for matrix functions ........ . .

E. O. Milton, Fourier transforms of odd and even tempered distributions ...

Dix Hayes Pettey, One-one-mappings onto locally connected generalized continua

Mark Bernard Ramras, Orders with finite global dimension

Doron Ravdin, Various types of local homogeneity. .

George Michael Reed, On metrizability of complete Moore spaces ...

Charles Small, Normal bases for quadratic extensions ..

Philip C. Tonne, Polynomials and Hausdorff matrices.... . . 